\title{
Aeromagnetic Surveying \\ Using a Simulated Unmanned Aircraft System
}

by

Raymond M. Caron

A thesis submitted to the Faculty of Graduate and Postdoctoral Affairs in partial fulfillment of the requirements for the degree of

Master of Science

in

Earth Sciences

Carleton University

Ottawa, Ontario

(C) 2011

Raymond M. Caron 


\section{Abstract}

Carleton University and Sander Geophysics are developing an unmanned aircraft system (UAS) with technologies to create a platform uniquely suited for aeromagnetic surveying. As an early indication of the expected performance of the UAS, a simulated UAS (sUAS) was built. The sUAS is a helicopter suspended T-shaped structure built with the same sensors and the same configuration as the UAS.

An $8.5 \mathrm{~km}^{2}$ area near Plevna, Ontario, was surveyed with the sUAS suspended 50 $m$ above ground. The survey area was chosen on the basis of its complex geological structure. The total magnetic intensity (TMI) data recorded was compared to that obtained during a conventional fixed-wing survey and a ground survey. Data from an experimental transverse magneto-gradiometer was also recorded by the sUAS and analyzed.

The sUAS TMI data shows a higher level of detail than the conventional fixedwing data, and a similar level of detail to that of the ground survey without any loss in data due to topography and inaccessible areas. The transverse magneto-gradiometer tested by the sUAS was found to adequately measure the gradient of the magnetic field. 


\section{Acknowledgements}

This dissertation would not have been possible without the guidance and the help of several individuals, and group of individuals, who in one way or another contributed and extended their valuable assistance in the preparation and completion of this study. First and foremost, my utmost gratitude to Dr. Claire Samson, my supervisor and Chair of the Department of Earth Sciences at Carleton University, whose guidance, assistance, and encouragement I will never forget. I would also like to express my gratitude to Luise Sander and Stephan Sander, of Sander Geophysics, who not only sponsored my research, but provided me with an opportunity to work with Sander Geophysics. I would like to thank: Dr. Martin Bates, of Sander Geophysics, who shared his knowledge and expertise on many occasions; Reed Archer, of Sander Geophysics, who assisted in many components of the sUAS and ground surveys; Steven Fergusson, of Sander Geophysics, for his assistance with my own project and for his ongoing support for the GeoSurv project, Dr. Paul Straznicky, GeoSurv II UAS Project Manager, who provided many opportunities to share my research; Lorne McKee and David Boteler, of the Ottawa Geomagnetic Laboratory who facilitated the ground survey along with Joel Dubé, of Sander Geophysics; and to Duncan Studd and Robert Forrester without whom the ground survey would not have been possible.

I would like to express a big thank you to the staff at Sander Geophysics, especially the geophysicists who let me feel welcome and provided their assistance, and the engineers who built the sUAS. I would also like to thank the research members of the GeoSurv II UAS project, and the entire Earth Sciences department at Carleton University. Last but not the least; I would like to thank my family for providing their encouragement and support throughout my studies. 


\section{Table of Contents}

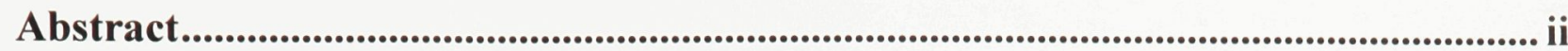

Acknowledgements .......................................................................................................................ii

List of Tables ....................................................................................................................... vi

List of Figures.................................................................................................................................. vii

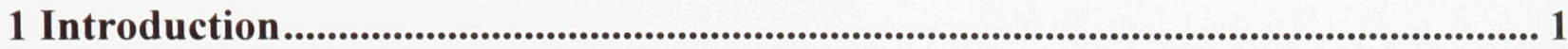

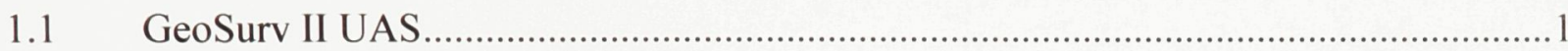

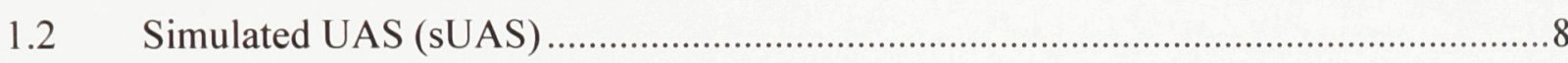

1.3 Research Objectives and Structure of the Thesis.................................................. 8

2 Background theory .......................................................................................................... 12

2.1 A Brief History of Magnetic and Aeromagnetic Surveying …................................ 12

2.2 Physical Principles and Underlying Method .................................................... 14

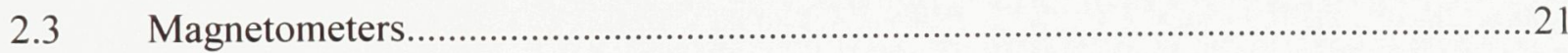

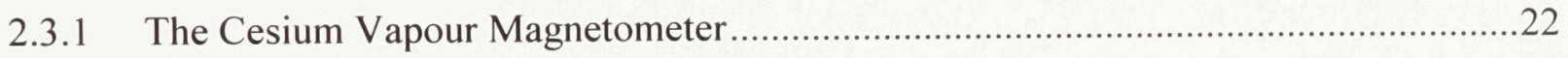

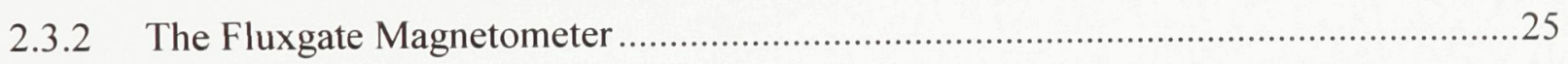

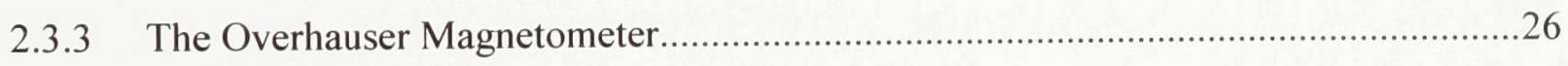

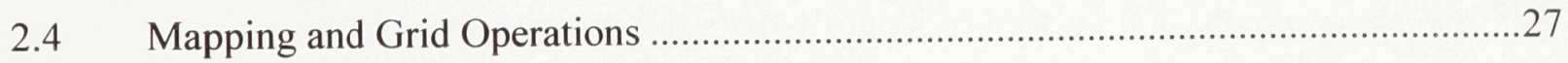

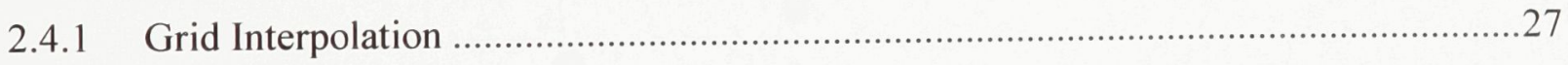

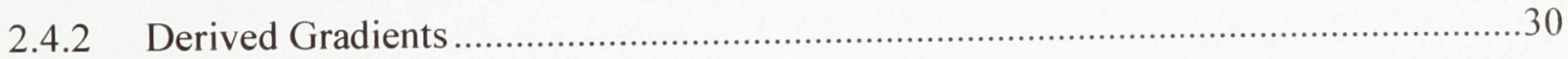

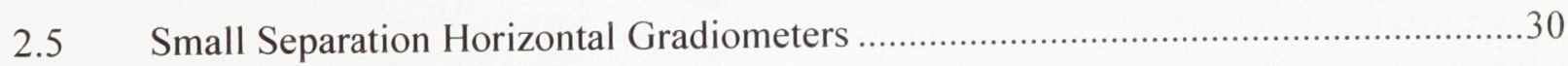

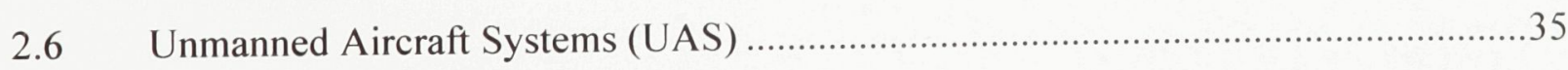

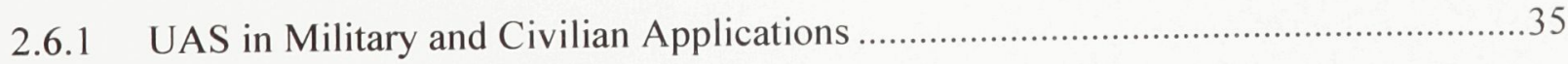

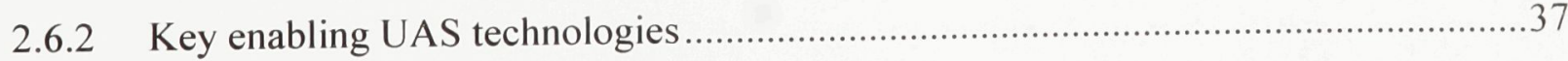

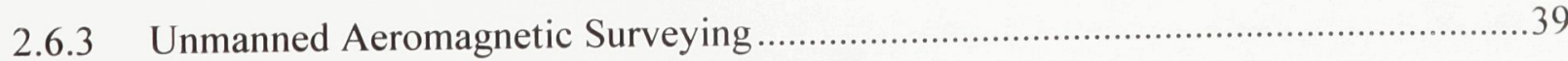


3 Survey Site 43

4 Data Acquisition and Processing ........................................................................ 51

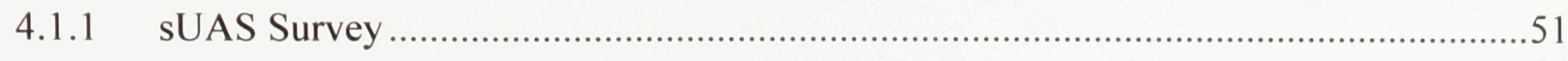

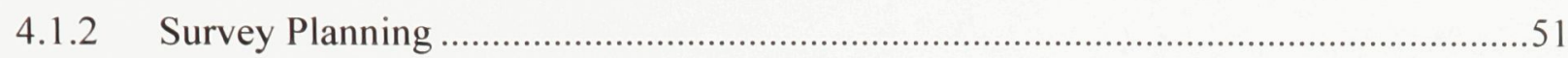

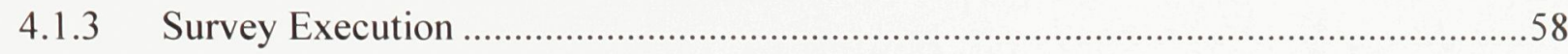

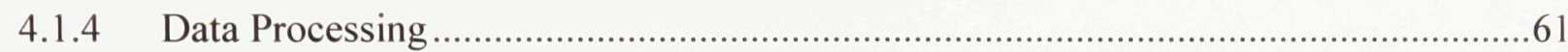

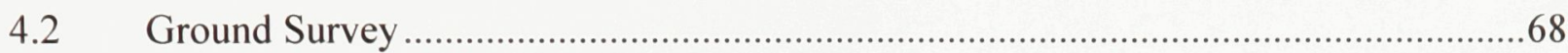

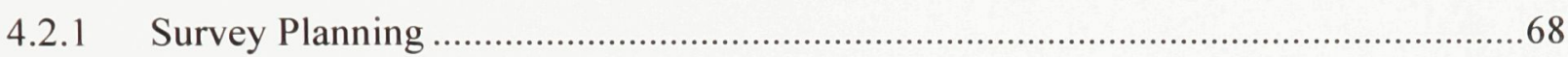

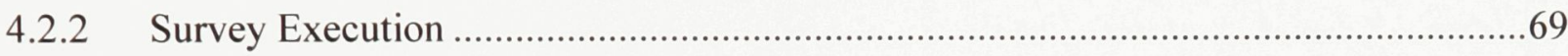

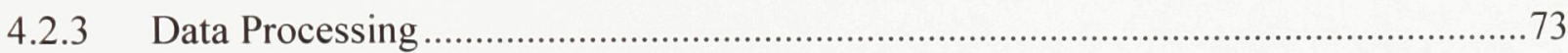

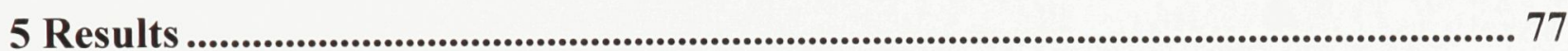

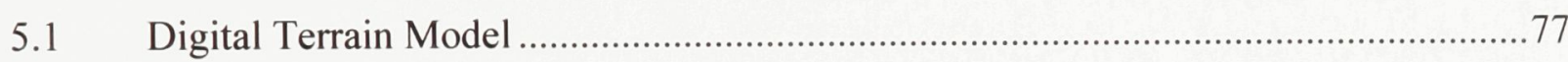

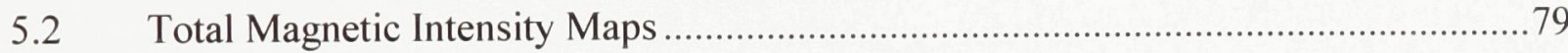

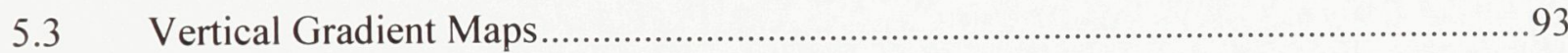

5.4 Transverse and Longitudinal Gradient Maps .....................................................96

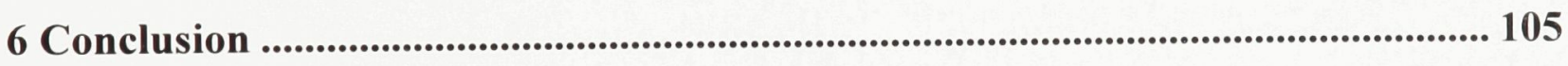

References ........................................................................................................ 108 


\section{List of Tables}

Table 1.1: Requirements to guide the development of the GeoSurv II UAS ................. 3

Table 2.1: Summary magnetometer specifications ............................................ 23

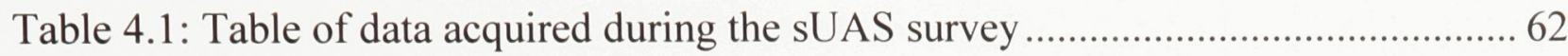

Table 4.2: Table of data acquired during the ground survey ….............................. 75

Table 5.1: Summary Table of Maps and Data Sets ................................................ 78 


\section{List of Figures}

Figure 1.1: Photograph of the Prototype UAS GeoSurv II ................................................. 5

Figure 1.2: Rendered Drawing of the GeoSurv II Prototype ............................................... 6

Figure 1.3: Rendered Drawing of the GeoSurv II Fuselage ………………….................. 7

Figure 1.4: Concept Drawing of the Simulated UAS ……..............................................

Figure 1.5: Simulated UAS Being Prepared for a Test Flight ......................................... 10

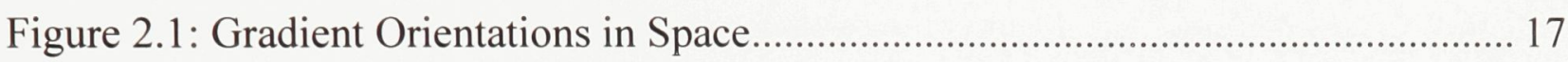

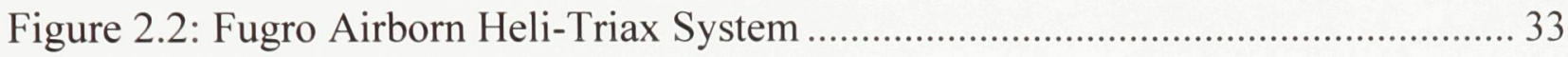

Figure 2.3: Fugro Airborn Heli-Triax Results .............................................................. 34

Figure 3.1: Satellite Image of Survey Site …………...................................................... 44

Figure 3.2: Structural Geology Map of the Region Around the Survey Site.................... 46

Figure 3.3: Distribution of Terrain Gradient in the Survey Site ....................................... 50

Figure 4.1: Shuttle Radar Map of Survey Site ………………......................................... 52

Figure 4.2: Figure of Terrain Clearance Comparison ...................................................... 55

Figure 4.3: Flight Drape Map of Survey Site …………............................................. 59

Figure 4.4: Photograph of the sUAS Being Flown Under a Helicopter ........................... 60

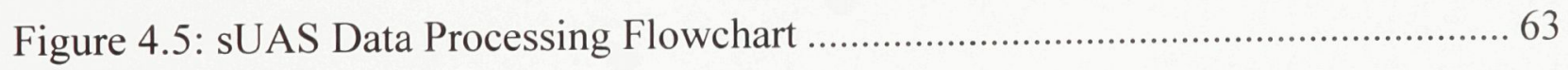

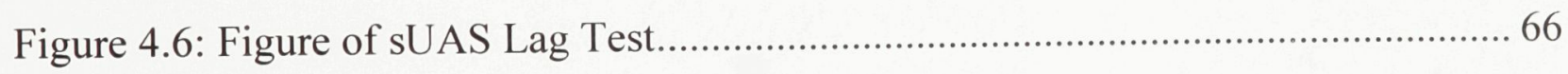

Picture 4.7: Photograph of the February Ground Survey ……....................................... 70

Picture 4.8: Photograph of the November Ground Survey ............................................. 71

Figure 4.9: Ground Survey Data Processing Flow Chart …………................................. 76

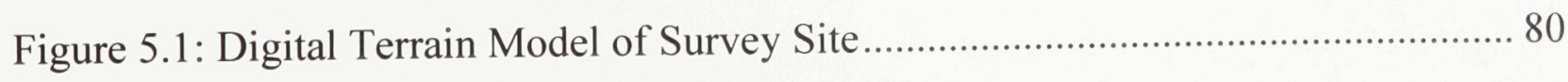




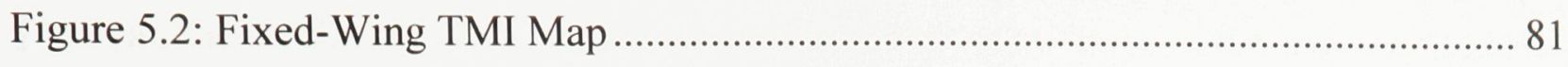

Figure 5.3: Structural Geology and Fixed-Wing TMI Map............................................ 82

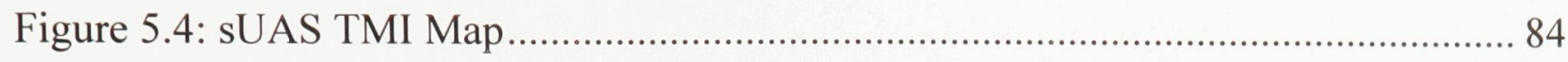

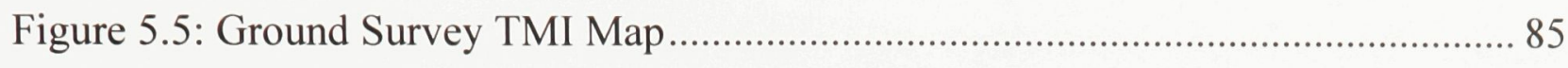

Figure 5.6: Sequence of Upward Continued Ground Survey TMI Maps .......................... 87

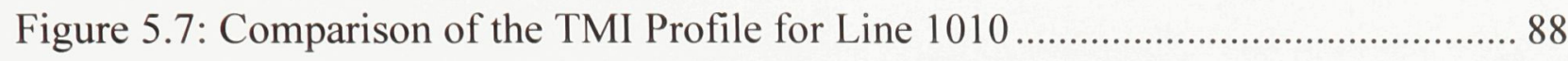

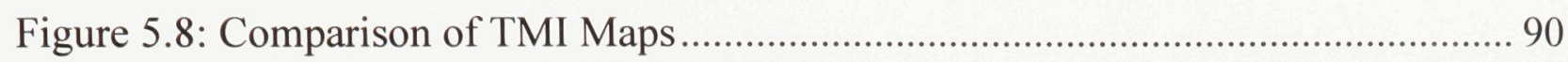

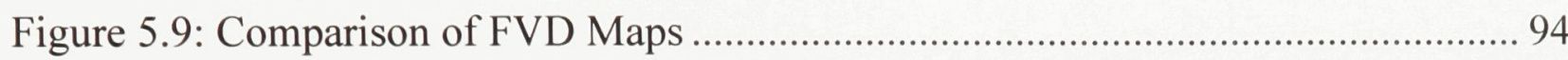

Figure 5.10: Comparison of Transverse Gradient with Transverse Derivative ................ 97

Figure 5.11: Comparison of Transverse Gradient with FVD …………........................... 99

Figure 5.12: Comparison of Transverse Gradient with Longitudinal Gradient.............. 101

Figure 5.13: Transverse Gradient Profile at Different Altitudes for Line 1010 ............. 103 


\section{Introduction}

\subsection{GeoSurv II UAS}

Aeromagnetic surveys are usually flown using manned aircraft such as the Navajo Piper or the Cessna Caravan. They are usually flown at a safe altitude which can be approximately $100 \mathrm{~m}$ or more in altitude over a rugged, forest covered, terrain. Aircraft crew comprises of a pilot and a co-pilot. Overall survey crew for a campaign in a remote area typically also includes a geophysicist and an aircraft maintenance engineer. In order to conduct an aeromagnetic survey, the aircraft needs to be modified to remove ferromagnetic materials from the proximity of the magnetometers which are often mounted on booms outboard the wingtips. The modification is required to reduce the amount of magnetic noise created by the aircraft and recorded by the magnetometers that are measuring Earth's magnetic field.

A demand exists for high-resolution, smaller size surveys flown at low altitudes (Barnard, 2008). Sander Geophysics Limited (SGL) and Carleton University (CU), both located in Ottawa, Canada, started collaborating in 2004 to assess the feasibility of unmanned aircraft systems (UAS) for such surveys. The SGL-CU collaboration involves teams of final-year undergraduate engineering students, from the departments of mechanical and aerospace engineering, electrical engineering, and systems and computer engineering, and graduate students from several disciplines. 
The SGL-CU initiative represents one of the first projects to design a UAS specifically for the civil application of aeromagnetic surveying. The overarching objective of the project is not simply to design a functional UAS from scratch, it is to integrate specific technologies to create an innovative platform that is uniquely suited to make high resolution aeromagnetic measurements. To achieve that goal the GeoSurv II UAS project is organized into a set of research initiatives including: low-cost composites, autonomous operation, obstacle detection and avoidance, magnetic signature control, and geomagnetic data acquisition. There is a dedicated research team developing technologies for each research initiative listed as part of the GeoSurv II UAS project. The primary requirements that must be demonstrated by the UAS are listed in Table 1.1.

The research and development initiative for low-cost composites involves the development of a light composite airframe that is non-magnetic and relatively cheap to manufacture compared to standard methods. The autonomous operation systems include navigation and telemetry. These systems are integrated with the object detection and avoidance system. Both systems are still under development. These integrated systems process information from the GPS, autopilot, navigation system, object detection and avoidance system, and the radar in order to achieve an accurate flight path with the ability to avoid both fixed and moving obstacles that could be situated in the air or on the ground. The magnetic signature control initiative is concerned with identifying and reducing sources of magnetic interference created by both electronic and ferromagnetic 
Table 1.1: Requirements to Guide the Development of the GeoSurv II UAS

\begin{tabular}{|c|c|}
\hline \multicolumn{2}{|r|}{ Some UAS Design Specifications } \\
\hline \multirow[t]{9}{*}{ Mechanical } & Airspeed Range of 30 to $51 \mathrm{~m} / \mathrm{s}$ (60 to $100 \mathrm{kts})$ \\
\hline & Endurance of 8 hours at $30 \mathrm{~m} / \mathrm{s}$ (60 kts) \\
\hline & Minimum flight altitude of $10 \mathrm{~m}$ above ground level \\
\hline & Maximum flight altitude of $2440 \mathrm{~m}$ above sea level \\
\hline & Payload capacity of $9.1 \mathrm{~kg}$ \\
\hline & Operational temperature range of $-45^{\circ}$ to $40^{\circ} \mathrm{C}$ \\
\hline & Can operate in all light weather conditions (rain, snow, wind) \\
\hline & Composite airframe \\
\hline & Non-magnetic fasteners \\
\hline \multirow[t]{2}{*}{ Aircraft magnetic noise } & Must be less than $0.01 \pm 0.005 \mathrm{nT}$ for time-varying sources $<5 \mathrm{~Hz}$ \\
\hline & Must be less than $0.1 \pm 0.05 \mathrm{nT}$ for fixed sources \\
\hline \multirow[t]{5}{*}{ Autonomous Control } & $\begin{array}{l}\text { Autopilot system that follows a flight plan that can be modified on } \\
\text { the fly }\end{array}$ \\
\hline & Object detection and avoidance system \\
\hline & System and survey telemetry updates via radio or satellite \\
\hline & GPS navigation \\
\hline & Radar and laser altimeter for guidance and terrain imaging \\
\hline \multirow[t]{5}{*}{ Survey } & Two Geometrics G822-A cesium resonance magnetometers \\
\hline & Wingtip to wingtip horizontal gradiometer \\
\hline & One Billingsley TFM 100 fluxgate magnetometers for compensation \\
\hline & Able to follow terrain up to a gradient of $10 \%$ \\
\hline & $\begin{array}{l}\text { Able to perform high altitude }(2440 \mathrm{~m}) \text { aircraft compensation } \\
\text { manoeuvres }\end{array}$ \\
\hline
\end{tabular}


sources within the aircraft. The geomagnetic data acquisition initiative is focused on data acquisition and processing, and the application of the GeoSurv II UAS for aeromagnetic surveying. This thesis is part of the geomagnetic data acquisition initiative of the of the GeoSurv II UAS project.

In March 2010, a prototype of the UAS was unveiled at Carleton University (Caron et al. 2011). Named the GeoSurv II, shown in Figure 1.1 and 1.2, the UAS is a twin-boom pusher aircraft with a wingspan of $4.9 \mathrm{~m}$. The UAS is not currently instrumented. Each yellow pod housed at the end of each wing is designed to contain one cesium resonance magnetometer for high resolution total magnetic intensity (TMI) measurements. There will also be one fluxgate magnetometer housed in the fuselage to provide data necessary for post-processing magnetic compensation of aircraft flight manoeuvres. The cesium magnetometers in each pod are separated by a horizontal distance of $4.67 \mathrm{~m}$. The horizontal gradiometer configuration of the cesium magnetometers will measure the transverse gradient of Earth's magnetic field relative to the heading of the aircraft. The airframe is made from a low-cost composite material that is manufactured using a closed cavity bag moulding variation of vacuum assisted resin transfer moulding (VARTM). The method is not commonly applied to airframe production, but was successfully modified to create low-cost high quality parts that can be used for airframe manufacturing (Maley 2008, Mahendran 2010). The fuselage, shown in Figure 1.3, of the UAS contains the computer electronics, batteries, and fuel. 


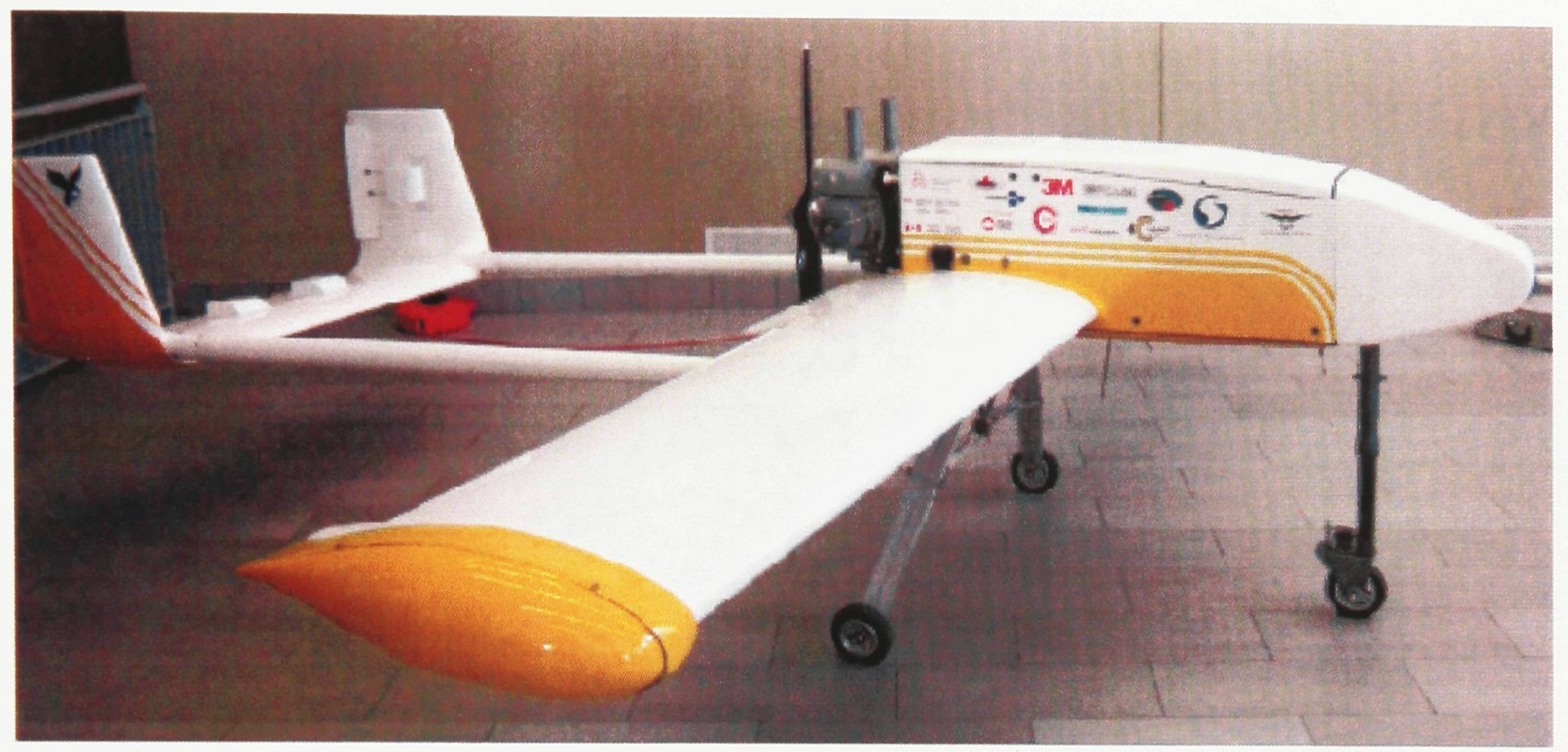

\section{Figure 1.1}

Photograph of the prototype UAS GeoSurv II being developed by Carleton University and Sander Geophysics. High resolution cesium resonance magnetometers are housed in the yellow pod at the end of each wing creating a magnetic gradiometer that measures the gradient of the magnetic field over a separation of $4.67 \mathrm{~m}$. The height of the aircraft with the landing gear is $1.22 \mathrm{~m}$. It is powered by a 2-stroke engine that has $30 \mathrm{HP}$ (Samson et al. 2010). 


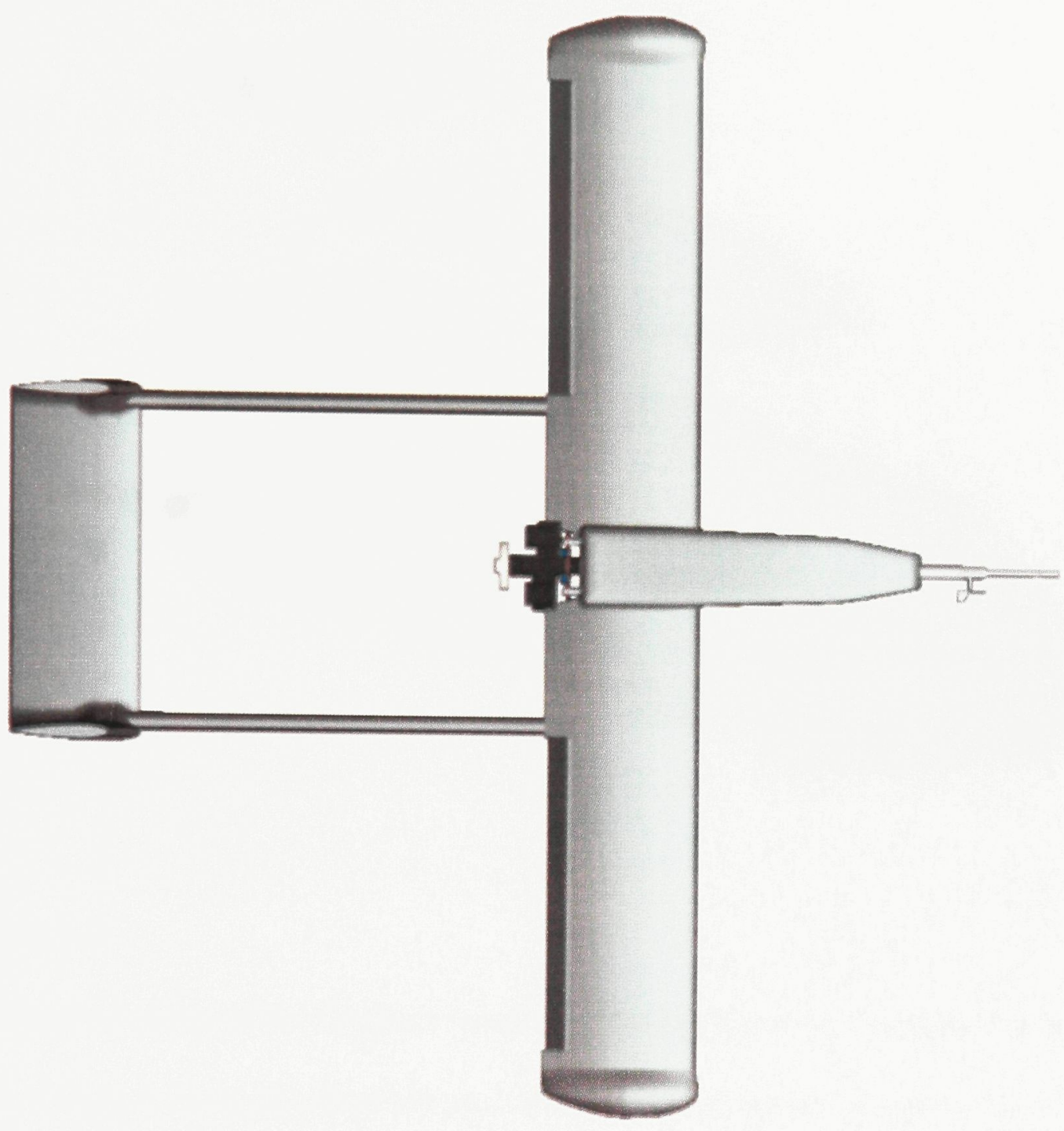

Figure 1.2

Rendered drawing of the GeoSurv II prototype (top view). The length of the aircraft from the tail to the nose, without the air data boom (to be used only in flight testing) is $4.27 \mathrm{~m}$. 


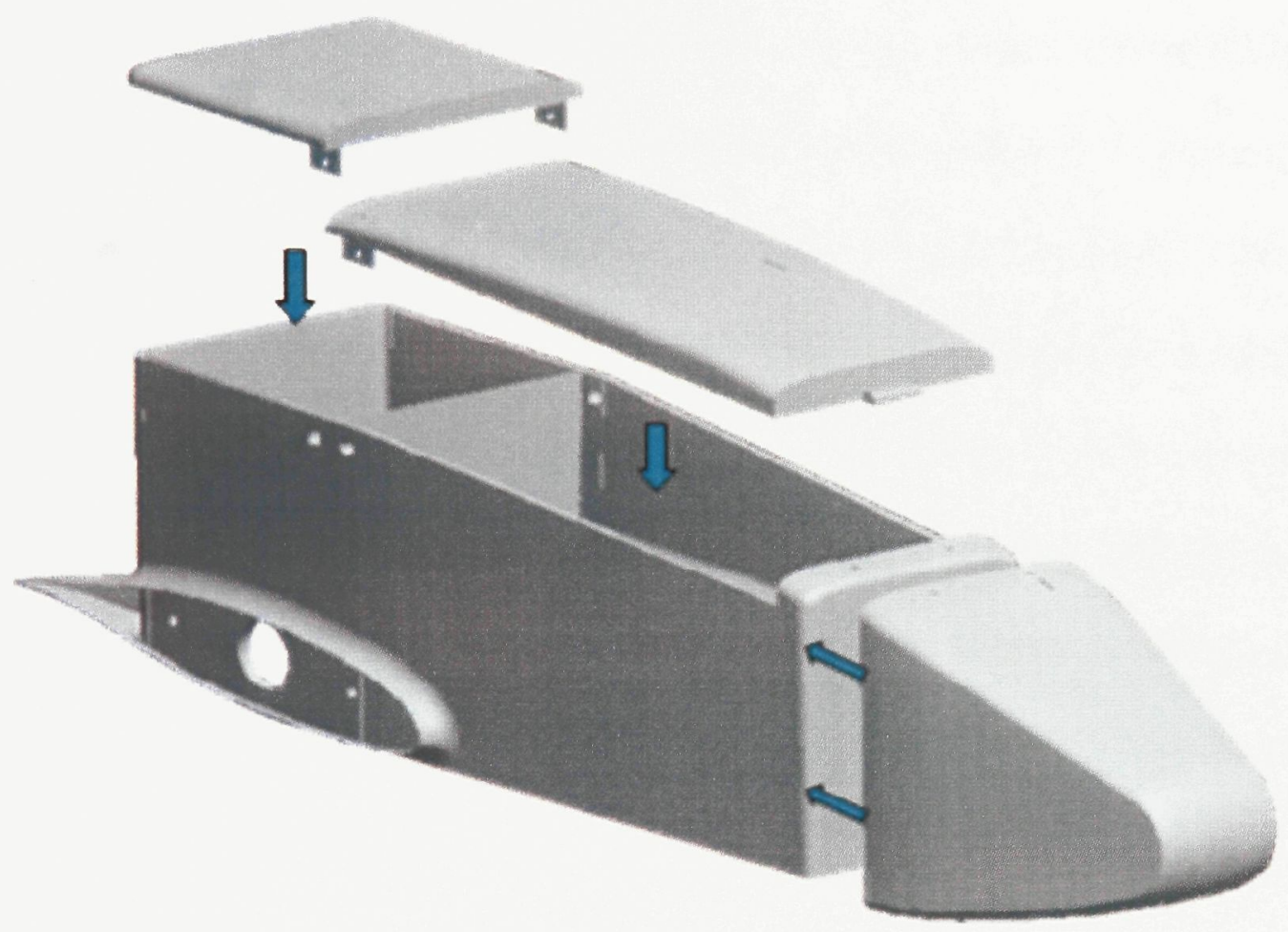

Figure 1.3

Rendered drawing of the GeoSurv fuselage. The fuselage is $1.1 \mathrm{~m}$ long, $0.3 \mathrm{~m}$ wide, and $0.39 \mathrm{~m}$ high. It is an all composite part with integrated inserts to manage local loads. It has a mass of $9.97 \mathrm{~kg}$ (Samson et al. 2010). 


\subsection{Simulated UAS (sUAS)}

While development of the GeoSurv II is continuing towards its first flight planned for fall 2011, a simulated UAS (sUAS) was built by Sander Geophysics in late 2010 to evaluate the aeromagnetic measurement capability of the future GeoSurv II UAS which includes the TMI and transverse gradient measurements and to facilitate the development of the object detection and avoidance system (Caron et al. 2011). The sUAS shown in Figures 1.4 and 1.5 is a T-shaped structure configured as a horizontal gradiometer with two cesium Geometrics G822A magnetometers spaced $4.67 \mathrm{~m}$ apart replicating both the wingspan and sensor geometry of the UAS. The sUAS is suspended $33 \mathrm{~m}$ beneath a helicopter to reduce the magnetic interference created by the aircraft to a negligible level (Caron et al. 2011). It records the Earth's magnetic field at the same resolution and altitude that the UAS is designed to achieve based on the topography and conditions of the area being surveyed.

\subsection{Research Objectives and Structure of the Thesis}

The focus of this thesis is to analyze the feasibility of using a sUAS as a tool to measure Earth's magnetic field. The primary objective is to determine the ability of the sUAS to detect small magnetic details created from geological structures, and to compare its performance against the conventional industry benchmarks of the fixed-wing aircraft and ground survey magnetic methods. To accomplish this objective, a study site was chosen to be surveyed using the sUAS. This survey data will then be mapped and 


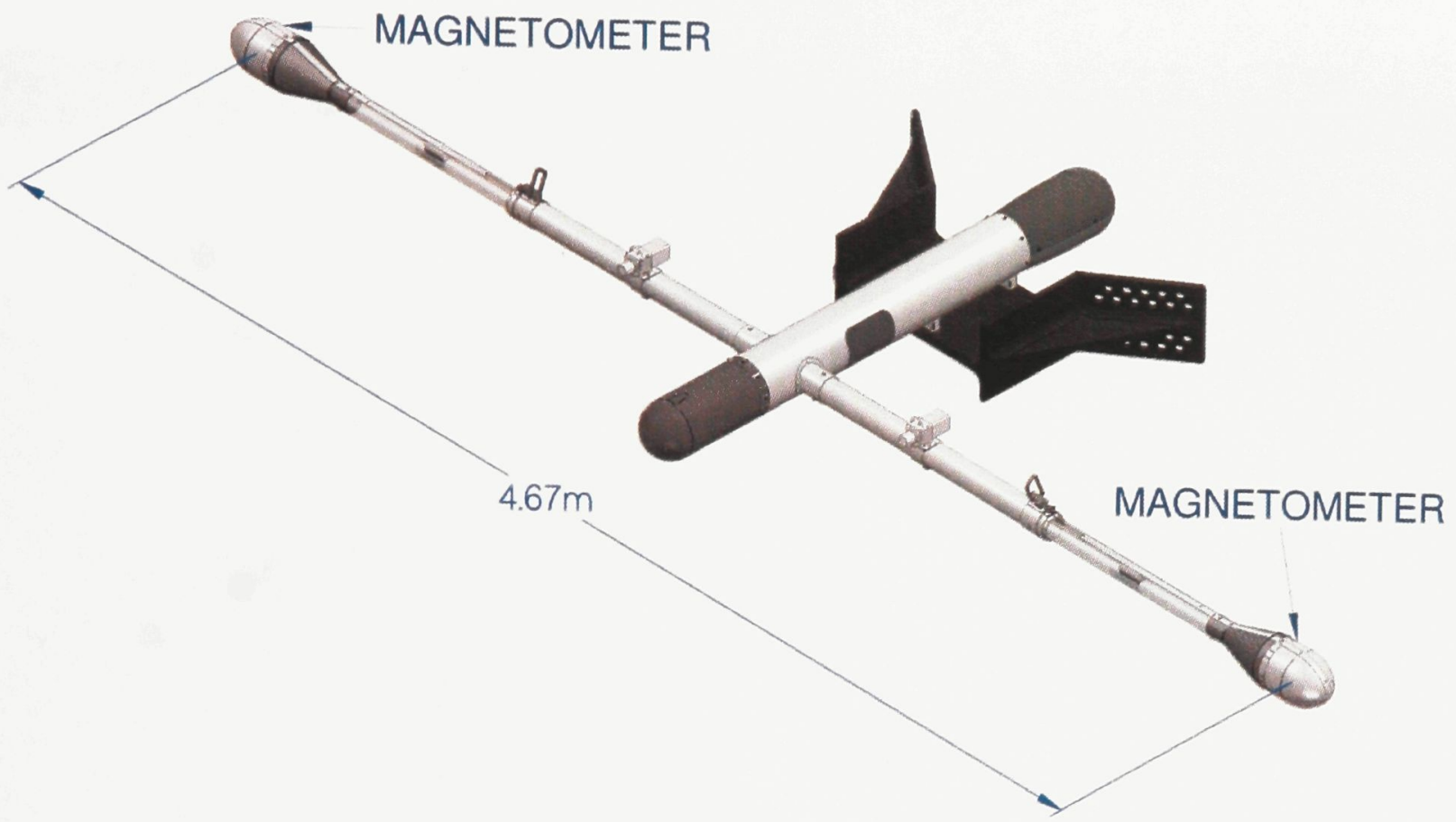

\section{Figure 1.4}

Concept drawing of the simulated UAS. The simulated UAS is a modified magnetic bird that can replicate the magnetic measurement capability of the UAS being developed. The sUAS has the same horizontal gradiometer configuration as the UAS, where the cesium magnetometers are separated by $4.67 \mathrm{~m}$. Fixtures for component testing, such as for the video camera used in the object detection and avoidance system, can be found internally and externally along the tubes separating the magnetometer pods, and within the central body of the magnetic bird. 


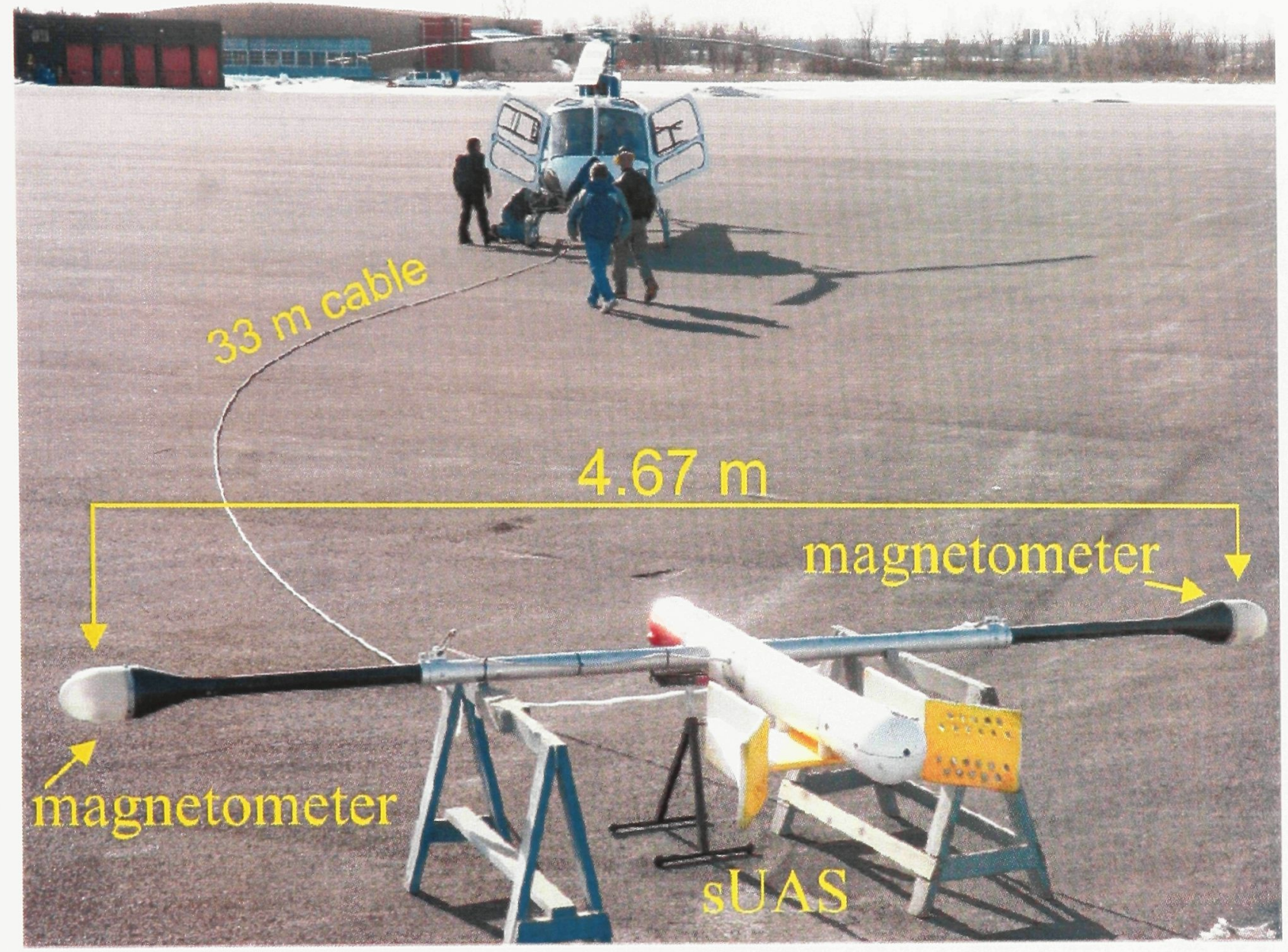

\section{Figure 1.5}

Simulated UAS being prepared for a test flight. 
compared by visual inspection to conventional fixed-wing survey data, and to mapped ground survey data.

A secondary objective is to determine if the magnetic gradiometer configuration being designed for the GeoSurv II UAS is capable of recording the transverse gradient of Earth's magnetic field, over the test survey site, at the altitude at which the UAS is designed to survey. This will be done by comparing the observed gradiometer data, acquired by the sUAS, with TMI calculated gradient data. As the sUAS is used as a proxy to evaluate the future capabilities of the GeoSurv II UAS, the results of these two objectives are considered to be an indication of the expected performance of the GeoSurv II UAS.

This thesis will briefly cover the history of magnetic and aeromagnetic surveying as well as some of the fundamental principles and underlying methods. The thesis will also briefly cover the history of UAS, the application of UAS to aeromagnetic surveying, and some of the enabling technologies that permit the application of UAS to aeromagnetic surveying. The thesis will go on to describe the survey site used to conduct the sUAS and the ground surveys. The surveying and data processing methods applied to the data from the sUAS and the ground surveys, will also be covered. The results will then be presented and discussed, followed by the conclusion of the study. 


\section{Background theory}

\subsection{A Brief History of Magnetic and Aeromagnetic Surveying}

The measurement of Earth's magnetic field in the hope of identifying an ore body is not a new technique. It is thought to have been first used successfully in Sweden in 1640 to identify iron ore deposits using a magnetic compass to observe the relative variations in the strength of the local magnetic field that were created by the presence of the deposits (Telford et al. 1990, Reynolds 1997). The first documented case of the use of a method to measure the relative strength of Earth's magnetic field in order to locate an ore body was by von Wrede in 1843 . The account was published in a book written by Thalén titled: The Examination of Iron Ore Deposits by Magnetic Measurements (Thalén 1879, Telford et al. 1990). Since then magnetic surveying has broadened from surveying for iron ore to a range of applications including surveying for heavy metals and platinum group elements, geological structures such as faults or folds, oil alteration zones, archaeological finds, unexploded ordinance, and shipwrecks associated with buried treasure.

Despite the advent of flight, magnetic surveying was, for a long time, restricted to the ground. This was a result of the delicate nature of the magnetometers that were in use. Instruments such as the torsion or balance type magnetometers, and the Schmidt variometer (Reynolds 1997) were generally limited to taking a measurement of a single component, usually the vertical, and had a limited accuracy of approximately $10 \mathrm{nT}$. 
They were highly sensitive to movement and so were not suitable for aeromagnetic surveying (Muffly 1946, Telford et al. 1990).

The first aeromagnetic survey was conducted using a simple apparatus called the double compass. The instrument was used on board the air ship Graf Zeppelin (Grotewahl 1930), and crudely identified the direction in which the Earth's magnetic field was deflected by a local anomaly (Holland 1876). Between 1936 and 1940, a system based on the induction coil, called the Earth Inductor Magnetometer, measured the absolute value of Earth's magnetic field. Its accuracy, however, was only on the order of $100 \mathrm{nT}$ (Lundak 1954, Hood 2007). The invention of the fluxgate magnetometer during the Second World War, marked the first widespread use of aeromagnetic surveying. It provided military officials with a method with which to scan large oceanic areas and detect hidden submarines in a quick and dependable fashion. Successful use of the fluxgate magnetometer led to the invention of new magnetometers such as the atomic resonance type and the quantum superconductor type that are able to record Earth's magnetic field at a much higher resolution.

The advent of high resolution magnetometers and the increased demand for reliable magnetic data for scientific and commercial use exposed resolution and accuracy problems. The problems of magnetic interference due to metallic aircraft components, diurnal variations of the magnetic field, lag corrections due to alternating directions of aircraft flight paths, and local variations of the global geomagnetic field had to be solved 
in order to accurately map magnetic anomalies of geological interest using the aeromagnetic method.

\subsection{Physical Principles and Underlying Method}

Earth's magnetic field can be loosely approximated by a bar magnet. Like a bar magnet, it has both a north and a south pole around which magnetic lines of force flow from a positive polarity in the north, to a negative polarity in the south. The magnetic field itself is an invisible field that acts upon objects that are ferrimagnetic, ferromagnetic, paramagnetic, and diamagnetic in composition. Ferrimagnetic materials, such as magnetite and ilmenite, have sub-lattices wherein the magnetic moments of the atoms are unbalanced resulting in a net magnetization of the material. Ferromagnetic materials are metals where the spin moment of an unpaired electron in the electron shell of an atom become coupled with other electrons orbiting other atoms of a compound, resulting in a net magnetization. A paramagnetic material is where the spin moment of unpaired electrons in an electron shell of an atom become partially coupled with the electrons of other atoms resulting in a weak net magnetization, compared to a ferromagnetic material. A diamagnetic material is when there are two paired electrons in an electron shell of an atom. In the presence of an external magnetic field the electrons adopt a spin moment that opposes the external field thereby creating a weak negative magnetic field (Reynolds 1997). 
The magnetic field is a vector that has a magnitude, referred to as the total magnetic intensity (TMI), and a direction. Both the magnitude and the direction of the force of a magnetic field can vary or be altered significantly by the presence of or introduction of a ferrimagnetic or ferromagnetic material. Since the magnetic lines of force flow from a positive pole to a negative pole, a negative pole will forcefully attract a positive pole and vice versa. Similarly, a negative or positive pole will also repel another negative or positive pole. The intensity with which the magnetic field repels or attracts is governed by Coulomb's Law and is expressed in equation 1 where $F$ is the force between poles $m_{l}$ and $m_{2}, \mu$ is the magnetic permeability of the medium separating the poles, and $r$ is the distance between the poles.

Equation 2.1: Coulomb's Law

$$
F=\frac{m_{1} m_{2}}{4 \pi \mu r^{2}}
$$

Equation 2.1 highlights an important characteristic of a magnetic field that has a large effect on aeromagnetic surveying. The force $\mathrm{F}$ of the magnetic field is inversely proportional to the square of the distance separating the magnetic poles. In terms of aeromagnetic surveying, the intensity of the magnetic field measured by the magnetometer falls as a square of the distance between the source and the magnetometer making the measurement (Reynolds 1997).

The fundamental principle of the magnetic method is to measure Earth's geomagnetic field and identify local changes within that field that are created by 
geological formations or structures. In a TMI survey, the magnetic field generated by geological formations can be isolated from the Earth's magnetic field by subtracting the International Geomagnetic Reference Field (IGRF) which approximates the magnetic field generated by Earth's mantle and core, and by subtracting high frequency diurnal variations. (Reynolds 1997)

The principle of the magnetic gradiometric method is to measure Earth's geomagnetic field at two points in space at the same time, and to calculate the spatial gradient of the magnetic field between those two points (Reynolds 1997). Measurements can be taken in either, or all, of the $\mathrm{X}$ (in the flight survey direction), $\mathrm{Y}$ (perpendicular to the flight survey direction), or $\mathrm{Z}$ (vertical) axes. A gradient measured along the $\mathrm{X}$ axis is the longitudinal gradient, a gradient measured along the $\mathrm{Y}$ axis is the transverse gradient, and the gradient measured along the $\mathrm{Z}$ axis is the vertical gradient. The concept is illustrated in Figure 2.1. Measurements taken in 1 or 2 of the 3 axes require assumptions about the pitch, roll, and yaw of the aircraft. In practice, without measuring all 3 axes of the magnetic field, a true longitudinal, transverse, and vertical gradient cannot be achieved; instead a relative gradient is measured where the gradient is a function of the attitude and orientation of the airplane at that moment during flight. The gradient between each measurement is calculated by subtracting a measurement made at one magnetometer by the measurement made at another magnetometer and dividing by the distance separating the two (Hood and Teskey 1989). The equations for calculating each gradient is shown in equations $2.2,2.3$, and 2.4 . 


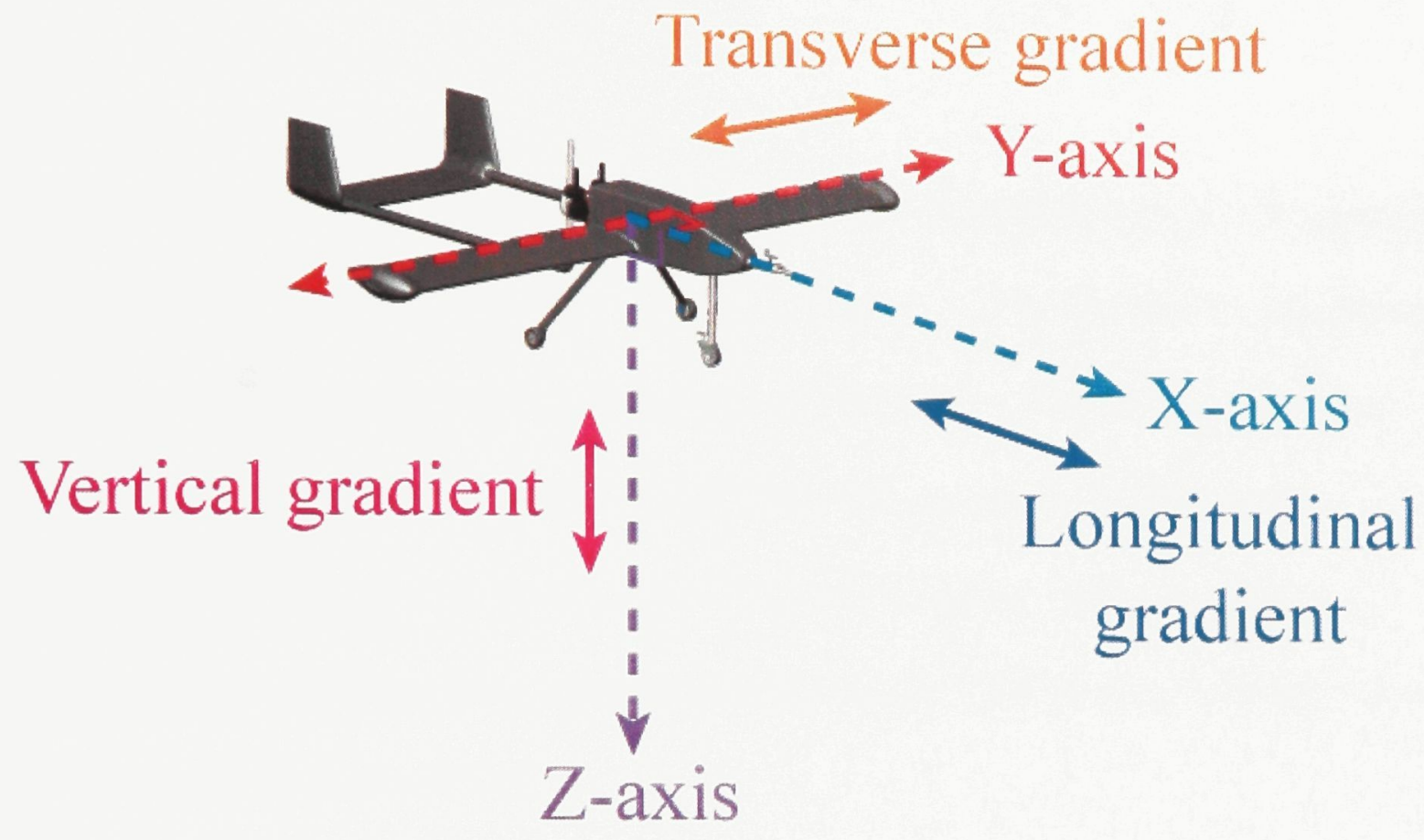

Figure 2.1

Relation between aircraft orientation, flight axes, and corresponding gradients. 
Equation 2.2: Longitudinal gradient ( $\mathrm{X}$ axis)

$$
\frac{\partial \Delta T}{\partial x}=\frac{\Delta T_{2}-\Delta T_{1}}{\Delta x}
$$

Equation 2.3: Transverse gradient ( $\mathrm{Y}$ axis)

$$
\frac{\partial \Delta T}{\partial y}=\frac{\Delta T_{2}-\Delta T_{1}}{\Delta y}
$$

Equation 2.4: Vertical gradient ( $Z$ axis)

$$
\frac{\partial \Delta T}{\partial z}=\frac{\Delta T_{2}-\Delta T_{1}}{\Delta z}
$$

Methods developed to calculate the longitudinal, transverse, and vertical gradients directly from the TMI data set has been shown to provide similar benefits as a measured gradient, but at a lower resolution (McMullan and McLellan 1997). Longitudinal and transverse gradients, calculated from a single TMI data set, are referred to as the longitudinal derivative and the transverse derivative, or together as the horizontal derivatives. The vertical gradient, calculated from a single TMI data set, is referred to as the first vertical derivative (FVD).

The calculation of the FVD, and to a lesser extent the horizontal derivatives, from the mapped TMI data has become standard throughout the geophysical industry. The calculation of a magnetic gradient requires the following assumptions; that the source geological structure is two dimensional, and that the TMI data set has been compensated, corrected, and levelled so that little noise remains (McMullan and McLellan 1997). The first assumption is a requirement of the mathematics used to calculate the approximation of the gradient. The second assumption is a by-product of the fact that magnetic noise can become amplified from the calculation of a gradient (McMullan and McLellan 1997). For measured gradients, no assumptions are required and so subsequent analytical techniques, 
such as the Euler deconvolution, can be considered more accurate due to the absence of any data bias created from the assumptions (McMullan and McLellan 1997).

The data recorded during gradiometric surveys are often considered to be superior to data gathered during a single-sensor TMI survey. The primary benefit of gradiometric surveying over TMI surveying is the ability to resolve detailed geological structures. In terms of geological resolution, the TMI data set is a sum of the local fields at a point in space, while the magnetic gradient measures the change of a spatial component of the magnetic field within an area of space. The TMI data set tends to provide the big picture, while the gradient data sets highlight high-frequency changes along a spatial axis (Hood and Tesky 1989). The higher frequency content of the gradiometric data set captures detailed variations in the magnetic field, especially changes that are orthogonal to the direction along which the gradient is measured. The gradiometric data can help resolve variations in the magnetic field that are created from smaller geological sources that are typically obscured in the TMI data set. The TMI data nevertheless remains an important source of information and is used along with the gradiometric data to provide a complete picture of the geology in a survey area (Hood and Tesky 1989). The second advantage of gradiometric surveying is the ability to correct for diurnal variations (Hood and Tesky 1989). Diurnal variations are fluctuations within the Earth's geomagnetic field that are caused by the contributions from time-varying magnetic fields. This interference can be created from the moon, tides, solar flares, cosmic radiation, and some cultural sources (Reynolds 1997). The problem is most severe in the auroral zone of Earth's magnetic field where the diurnal variations that are created by the solar wind and cosmic rays are 
more pronounced and variable over shorter distances (Reynolds 1997). When conducting a TMI survey, the diurnal variations are recorded by the magnetometer along with the rest of the magnetic field. In order to correct for these variations, they are also measured by a magnetometer that is fixed in space, such as at a ground station. The variations recorded by the ground station magnetometer can then be subtracted from the survey data set to obtain a data set that is free from time-varying effects. For large surveys that cover $500 \mathrm{~km}^{2}$ or more, setting up many different ground stations to provide corrections becomes expensive and impractical. The gradiometric method solves this problem as each sensor records the same diurnal variations at the same time. When the data from one sensor is subtracted from the data recorded from the other sensor, the diurnal variations are subtracted from the data (Hood and Tesky 1989).

Limitations to gradiometric surveying do exist. These limitations are a result of errors and noise introduced into the data set of each magnetometer used to calculate the gradient. There are several sources of error and noise. Sources of error include the magnetometer, and sources of noise include aircraft compensation, and magnetic eddies (Hardwick 1984, Sander and Moul 2004). Errors created from the magnetometer can be due to instrumental bias, differences in sensor orientation to the magnetic field, and instrument errors in counting the total field. These sources of error will vary from magnetometer to magnetometer. Noise introduced during aircraft compensation can be created from the difference between the estimated and actual magnitude of magnetic interference being applied to the magnetometer by the aircraft. This source of noise will also vary between magnetometers. Noise created due to magnetic eddies can vary in both 
space and time. Each magnetometer may be influenced to a different extent by the magnetic eddies occurring around the aircraft during surveying. Each of these sources of error and noise are considered to be inconsequential for a TMI data set due to their relatively small magnitude. For a gradiometric data set, where measurements from two magnetometers are used to determine a gradient, these sources of error and noise are compounded, and can influence the measured gradient or even exceed the magnitude of the measured gradient (Hardwick 1984, Sander and Moul 2004).

\subsection{Magnetometers}

The GeoSurv II UAS will be equipped with a fluxgate and two cesium magnetometers. The sUAS used to simulate the GeoSurv II was equipped with the same model cesium magnetometers, but not with a fluxgate magnetometer. A fluxgate magnetometer was not required because the sUAS is magnetically inert. The sUAS was also used outside of the envelope of magnetic interference created by the helicopter, and so magnetic compensation of the recorded data set was not required.

This section will introduce the cesium magnetometers being used on the sUAS, the fluxgate magnetometer that will be used in the UAS, and the proton precession magnetometer that was used during the ground survey that provided a magnetic data set for comparison. Table 2.1 provides a comparison summary of the specifications of each type of magnetometer. 


\subsubsection{The Cesium Vapour Magnetometer}

The cesium vapour magnetometer is a part of the alkali vapour group of magnetometers which are also atomic resonance type instruments. The instrument was first developed in 1957 (Dehmelt 1957, Bell and Bloom 1957, Bloom 1962), and can be designed to work with a number of different alkali gases. The cesium vapour magnetometer uses optical pumping and the Larmor response of cesium gas to an external magnetic field to measure the intensity of the magnetic field. When a charged particle is subjected to an external magnetic field, it precesses about the vector of the magnetic field. Alkali atoms such as the cesium atom, are suitable to measure this effect because they have a single electron in the outer shell with energy sublevels that are suitably spaced (Telford et al. 1990). Under normal conditions cesium atoms exist in one of eight energy sub-levels, in equal quantities. An important difference between these sub-levels is the spin axis of the single electron in the outer shell of the atom. To simplify the science, the following explanation will assume that the cesium atom only has 2 energy sub-levels (Dehmelt 1957, Bloom 1962, Telford et al. 1990, Reynolds 1997, Reeves 2005).

With 2 energy sub-levels, the electron in the outer shell of the cesium atom can have a precession spin axis that is either parallel, energy level A1, or anti-parallel, energy level A2, to an external magnetic field. In optical pumping, a circularly polarized photon, emitted by a cesium light source, is sent into the absorption chamber and absorbed by the electron in A1 pumping the electron, and the cesium atom, to a new and higher energy 
Table 2.1: Summary Magnetometer Specifications

\begin{tabular}{|c|c|c|c|}
\hline & \multicolumn{3}{|c|}{ Magnetometer } \\
\hline & $\begin{array}{c}\text { Geometrics Cesium } \\
\text { Vapour G-822A }\end{array}$ & $\begin{array}{c}\text { Billingsley Fluxgate } \\
\text { TFM100-G2 }\end{array}$ & $\begin{array}{c}\text { GEM Overhauser } \\
\text { GSM-19 }\end{array}$ \\
\hline Resolution & $0.001 \mathrm{nT}$ & $\mathrm{InT}$ & $0.01 \mathrm{nT}$ \\
\hline Sample Rate & $70-350 \mathrm{kHz}$ & $500 \mathrm{~Hz}-4 \mathrm{kHz}$ & $5 \mathrm{~Hz}$ \\
\hline Operation Range & $0.02-0.1 \mathrm{mT}$ & $\pm 0.1 \mathrm{mT}$ & $0.02-0.12 \mathrm{mT}$ \\
\hline Gradient tolerance & None & Yes, $0.5 \mathrm{mT}$ & Yes, $0.01 \mathrm{mT}$ \\
\hline $\begin{array}{l}\text { Orientation } \\
\text { Required }\end{array}$ & Yes ( $45^{\circ}$ to field $)$ & No & No \\
\hline Measures & Total Field & Relative Field & Total Field \\
\hline $\begin{array}{l}\text { Component } \\
\text { Measured }\end{array}$ & None & $\mathrm{X}, \mathrm{Y}, \mathrm{Z}$ & None \\
\hline Power Requirements & $24 \mathrm{VDC}$ at $750 \mathrm{~mA}$ & $15 \mathrm{VDC}$ at $25 \mathrm{~mA}$ & $12 \mathrm{VDC}$ at $200 \mathrm{~mA}$ \\
\hline Dimensions & $\begin{array}{c}158 \mathrm{~mm} \times 60 \mathrm{~mm} \\
\text { diameter }\end{array}$ & $\begin{array}{c}35 \mathrm{~mm} \times 32 \mathrm{~mm} \times 83 \\
\mathrm{~mm}\end{array}$ & $\begin{array}{c}175 \mathrm{~mm} \times 75 \mathrm{~mm} \\
\text { diameter }\end{array}$ \\
\hline Weight & $339 \mathrm{~g}$ & $100 \mathrm{~g}$ & $1.1 \mathrm{~kg}$ \\
\hline
\end{tabular}


level B. Once in the energy level B, the electron is no longer absorbing photons and so more photons pass through the gas to be detected by the photocell on the other side. The atoms in A2 do not absorb the light energy because the electrons precess in a direction that is not conducive to absorbing the polarized light energy. When the electrons in the higher energy level B use up their energy, they have an equal chance of returning to either sub-level A1 or A2, but if they return to A1 they'll get re-energized and pumped back to energy level B. This process over-populates energy sub-level A2 so that few atoms exist in energy sub-level A1. (Dehmelt 1957, Bloom 1962, Ando 1965, Telford et al. 1990, Reynolds 1997, Reeves 2005)

In the presence of an external magnetic field, the cesium atoms will precess about the vector of the magnetic field at the Larmor frequency that is equal to the intensity of the magnetic field. If an electromagnetic field is then transmitted, at a specific frequency, into the gas with the result of pushing the electron from energy sub-level A2 to energy sub-level A1, the pumping process can repeat itself. The frequency where all of the atoms are pushed to energy sub-level A1, will result in the light being absorbed by the cesium atoms, and the lowest amount of light will pass through the chamber to be detected by the photocell. This frequency will be a function of the Larmor precession frequency of the cesium atom and can be converted into a value that corresponds to the intensity of the total magnetic field. The magnetometer must be oriented at an angle of $45^{\circ}$ to Earth's magnetic field in order to accurately sample the magnetic field. (Dehmelt 1957, Bloom 1962, Ando 1965, Telford et al. 1990, Reynolds 1997, Reeves 2005) 
The model used for the sUAS survey is the Geometrics G-822A Cesium Magnetometer. It has a resolution of $0.001 \mathrm{nT}$ and a sampling rate that is equivalent to the Larmor frequency and can change from 70 to $350 \mathrm{kHz}$ (Geometrics 2004). This is the industry standard type of magnetometer used for aeromagnetic surveying and is comparable in resolution to other cesium vapour magnetometers built by other manufacturers.

\subsubsection{The Fluxgate Magnetometer}

The fluxgate was invented by Vacquier in 1940. It is a magnetic saturation type magnetometer which is relatively small with no moving parts. It works by winding a primary coil around two identical ferromagnetic bars in opposite directions. A secondary coil is then wound around both bars. An AC current is passed through the primary coil creating a time varying magnetic field. This time-varying field induces a secondary magnetic field within the ferromagnetic bars. Because the primary coil is wound around each bar in opposing directions, the induced magnetic fields are of equal intensity and opposing polarity at any point in time during the $\mathrm{AC}$ cycle, and so no current is generated in the secondary coil. In the presence of an external magnetic field this balance is interrupted. The external field will have the same polarity as one of the two induced fields. The fields with the same polarity will add up to an intensity that is higher than that of the opposing field. The stronger magnetic field will then induce a current in the secondary coil. The current generated is proportional to the strength of the external magnetic field. 
The model being used in the GeoSurv II UAS is a Billingsley TFM100-G2 Ultra Miniature Triaxial Fluxgate Magnetometer. It is a unit that can measure the relative intensity of the magnetic field along the $\mathrm{X}, \mathrm{Y}$, and $\mathrm{Z}$ axes simultaneously. It has a resolution of $1 \mathrm{nT}$, a weight of 100 grams, and is less than $8.3 \mathrm{~cm}$ in size (Billingsley Aerospace \& Defense 2008). The unit will be installed in the fuselage of the GeoSurv in order to measure the change in the magnetic field during flight manoeuvres and along different headings. The data recorded will be used to correct the higher resolution data gathered by the cesium magnetometers in a process called compensation.

\subsubsection{The Overhauser Magnetometer}

The Overhauser magnetometer is a type of, and improvement on the design of, the proton free-precession magnetometer. It measures the total magnetic intensity of Earth's magnetic field. It works through the interactions of two elements in a chamber. A freeradical element in liquid state that has an unpaired electron in an electron shell is mixed with hydrogen atoms. Both the free-radical and hydrogen atoms precess about the vector of a magnetic field at a Larmor frequency that is proportional to the strength of the magnetic field. When an external magnetic field is transmitted through the chamber, the hydrogen and free-radical atoms will precess about the vector this magnetic field. An Overhauser effect is then shared between the free-radical protons and the hydrogen protons. The Overhauser effect works to magnify the precession frequency among all of the atoms by increasing the strength of the polarization between protons. When the 
external magnetic field is shut-off, the hydrogen and free-radical atoms will begin to precess about Earth's magnetic field. Another Overhauser effect is then shared between the hydrogen protons and the free-radical protons. Because protons are charged particles they generate a voltage, within a coil wrapped around the chamber, as they precess at the Larmor frequency. The voltage fluctuates at the Larmor frequency and can be measured (Waters and Frances 1958, Telford et al. 1990, Hrvoic and Hollyer 2002).

The Overhauser magnetometer used in the ground survey is the GEM Overhauser GSM-19 Walking Magnetometer. It has a resolution of $0.1 \mathrm{nT}$ and can take measurements of the field every $0.2 \mathrm{~s}$ (GEM Systems 2008). Unlike the cesium vapour magnetometer, the Overhauser magnetometer can take reliable measurements without the requirement of sensor orientation to the vector of the magnetic field. It can be mounted on a backpack with a setup that is designed for ground surveying by uninterrupted walking. It was chosen to perform the ground survey due to its ease of use and its ability to measure the total magnetic intensity.

\subsection{Mapping and Grid Operations}

\subsubsection{Grid Interpolation}

A common challenge with all digital data-sets is the sampling rate and area over which the data was gathered. With respect to magnetics, there are two problems associated with the sampling rate of a measurement during a survey. The first problem relates to the size of the data set itself. As it is preferable to record measurements as often 
as possible during a survey, a large data set is often created that in many cases may exceed the size of the memory set aside to record it. On the other hand, if an insufficient sampling rate is chosen, then the data set may not have sampled a sufficient amount of the high frequency content of the magnetic field. Both of these two concerns need to be balanced in order to generate a data set that is representative of the magnetic field being measured, while at the same being generating a data set small enough to fit into the available memory.

The concerns associated with the area over which the data is gathered is more of a monetary problem. As variations in Earth's magnetic field are known to be small, depending on the distance from the source, measurements are often taken every few meters along a particular direction. In order to completely sample an area to the same extent, measurements should be taken with the same separation in both the $\mathrm{X}$ and $\mathrm{Y}$ directions. A high number of measurements requires a longer time to acquire, which is costly. In order to decrease the cost of a survey, gaps are introduced into the survey grid creating survey lines. These survey lines are designed to run parallel to each other, with a specified gap between each line. The larger the gap, the fewer the survey lines and the fewer measurements required in order to cover a survey area. A decreasing number of survey lines, and a lower amount of measurements taken, reduce the resolution of the survey by omitting high frequency content between survey lines. The gaps between lines are interpolated to create a grid that covers the survey area. 
In aeromagnetic surveying the most common method to interpolate the gaps between survey lines is the minimum curvature method. First introduced by Ian Briggs in 1974 (Briggs 1974). Minimum curvature is a two dimensional, in the $\mathrm{X}$ and $\mathrm{Y}$ directions, spline type interpolation method. It works by interpolating between measured data values following a grid pattern, and assigning a value to a grid cell based on a weighted twodimensional convolution calculation. The method makes several interpolation passes over the grid, with each sequential pass at a finer detail than the last (O'Connell et.al. 2005). The grid cell size is determined by the spacing between the survey lines. Along a survey line, depending on the size of the grid cell and the spatial sampling rate of the measurements, a grid cell may represent the average of multiple measurements. A small grid cell size will have each individual cell covering a smaller area. This will result in each cell representing an average value of smaller number of measurements. An average created from a smaller sample will result in a greater amount of high frequency features in a map. A small grid cell size may also introduce interpolation error, where incorrect features may be created, such as with the boudinage and bulls-eye artifacts that result from spatial aliasing (Reeves 2005, O’Connell et.al. 2005). To reduce spatial aliasing errors, a larger grid cell size is chosen. The aeromagnetic industry typically follows a ratio of line spacing to cell size of 1:4 or 1:5 in order to produce a map that has an acceptable level of resolution with a low amount of spatial aliasing errors (Reeves 2005). 


\subsubsection{Derived Gradients}

The technique to calculate a gradient can be accomplished using one of two methods. The first method calculates the gradient by applying a discrete Fourier (DF) transform on the data. The data is then filtered based on its wavelength and transformed back into the time-domain to obtain the result (Paine 1986, Telford et al. 1990). The second method is applied in the space domain, where a convolution filter is applied to the data points using a chosen coefficient that has a spectral characteristic that matches the continuous operator of the grid (Paine 1986, Telford et al. 1990). Each method returns comparable but different results, and each method has its own advantages based on the situation within which it is applied. Both methods have been demonstrated to correlate with the measured gradient. There are examples where the measured gradient has shown to be more accurate (McMullan and McLellan 1997). Derived gradients also have inherent limitations that are created from the TMI map from which they are derived. The interpolation method, the grid cell size, sampling rate, and survey line spacing, can all act to reduce the high frequency content of the derived gradient (McMullan and McLellan 1997). In this respect derived gradients can only be as accurate as the map from which they were derived.

\subsection{Small Separation Horizontal Gradiometers}

The use of a horizontal gradiometer with configuration of less than 5 meters between sensors is not new to airborne surveying. It is well documented in studies 
detailing advances in surveying for unexploded ordinance. For this application, the separation between sensors can be small. An example of such a system is the AMTADS system where the magnetometers are separated by $1.5 \mathrm{~m}$ (Billings and Wright 2009). In surveying for unexploded ordinance, the target is small, $81 \mathrm{~mm}$ in size for a mortar which is generally on top of the ground or buried within a few meters of the surface. In order to sample such a small target, the surveying helicopter is required to fly 1 to $3 \mathrm{~m}$ above ground (Billings and Wright 2009).

The use of a horizontal gradiometer with a small separation at higher altitudes was tested by Fugro Airborne Surveys. The Heli-Triax was developed for aeromagnetic surveying to locate weak magnetic anomalies, such as those associated with a small pipeline, in the Western Canada Sedimentary Basin (WCSB) (Mushayandebvu and Davies 2006). The Heli-Triax is a system equipped with 4 cesium magnetometers and is designed to be used while suspended $30 \mathrm{~m}$ beneath a helicopter. Two magnetometers are arranged as a horizontal $\mathrm{Y}$-axis transverse gradiometer that has a sensor separation of 3 $\mathrm{m}$. The third magnetometer is located mid-distance between, and 3 meters beneath, the other two. The fourth magnetometer is located in the center, and a few meters behind the two transverse magnetometers. The average of the measurements taken at the two horizontal gradiometers can be used to calculate the vertical gradient using the third magnetometer, and the longitudinal gradient using the fourth magnetometer. There is an illustration of the system in Figure 2.2. 
The system was tested over the WCSB during the Ferrier survey, flown at a nominal altitude of $30 \mathrm{~m}$, and a line spacing of $100 \mathrm{~m}$. The results were compared against conventional aeromagnetic surveys flown by fixed-wing aircraft that were conducted with a line spacing of 400 to $800 \mathrm{~m}$ (Mushayandebvu and Davies 2006). The comparison of the two systems using the Ferrier survey showed that the TMI results of the lower altitude Heli-Triax survey were of much higher resolution than the higher altitude fixedwing survey (Mushayandebvu and Davies 2006). The report did not identify if the source of the high frequency magnetic trends, which are illustrated in the Heli-Triax TMI map, originated from the basement or the sedimentary rock.

The Heli-Triax data gathered from the Ferrier survey was analyzed by comparing the results of the measured gradients, with the TMI derived gradients. The results, in Figure 2.3, show that the measured gradient maps are of lower quality than the derived gradient maps. The measured gradients have more high frequency signal content, and a greater amount of noise compared to the derived gradient maps. The noise appears as a dithering across the maps and which acts to obscure the magnetic trends. These same trends are well illustrated in the derived gradient maps (Mushayandebvu and Davies 2006). The report is disappointing as the maps made from the measured gradients did not resolve magnetic trends with an accuracy as high as the calculated gradients. The transverse data set is also found to have the highest amount of noise, and is also the map 


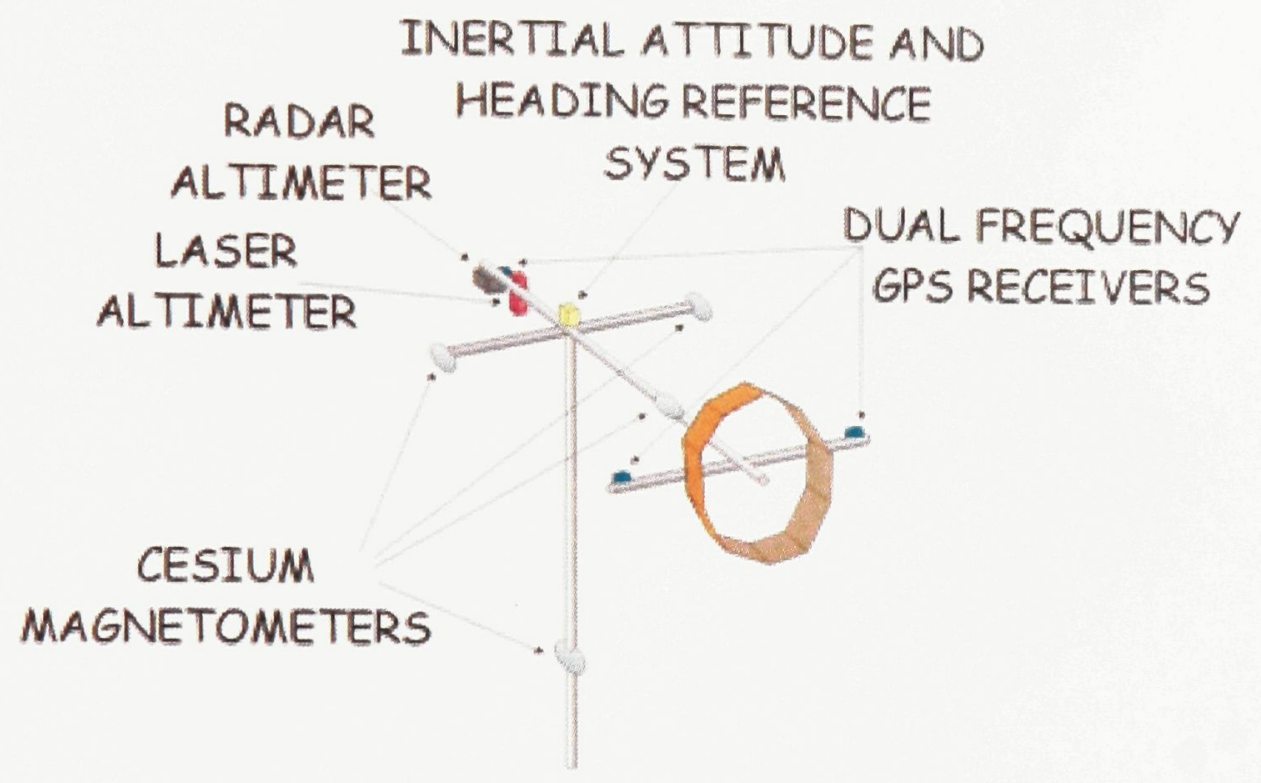

Figure 2.2

Fugro Airborne Surveys Heli-Triax system. System is used for aeromagnetic surveying. Horizontal and vertical magnetometers are separated by $3 \mathrm{~m}$. Figure modified from Mushayandebvu and Davies 2006. 


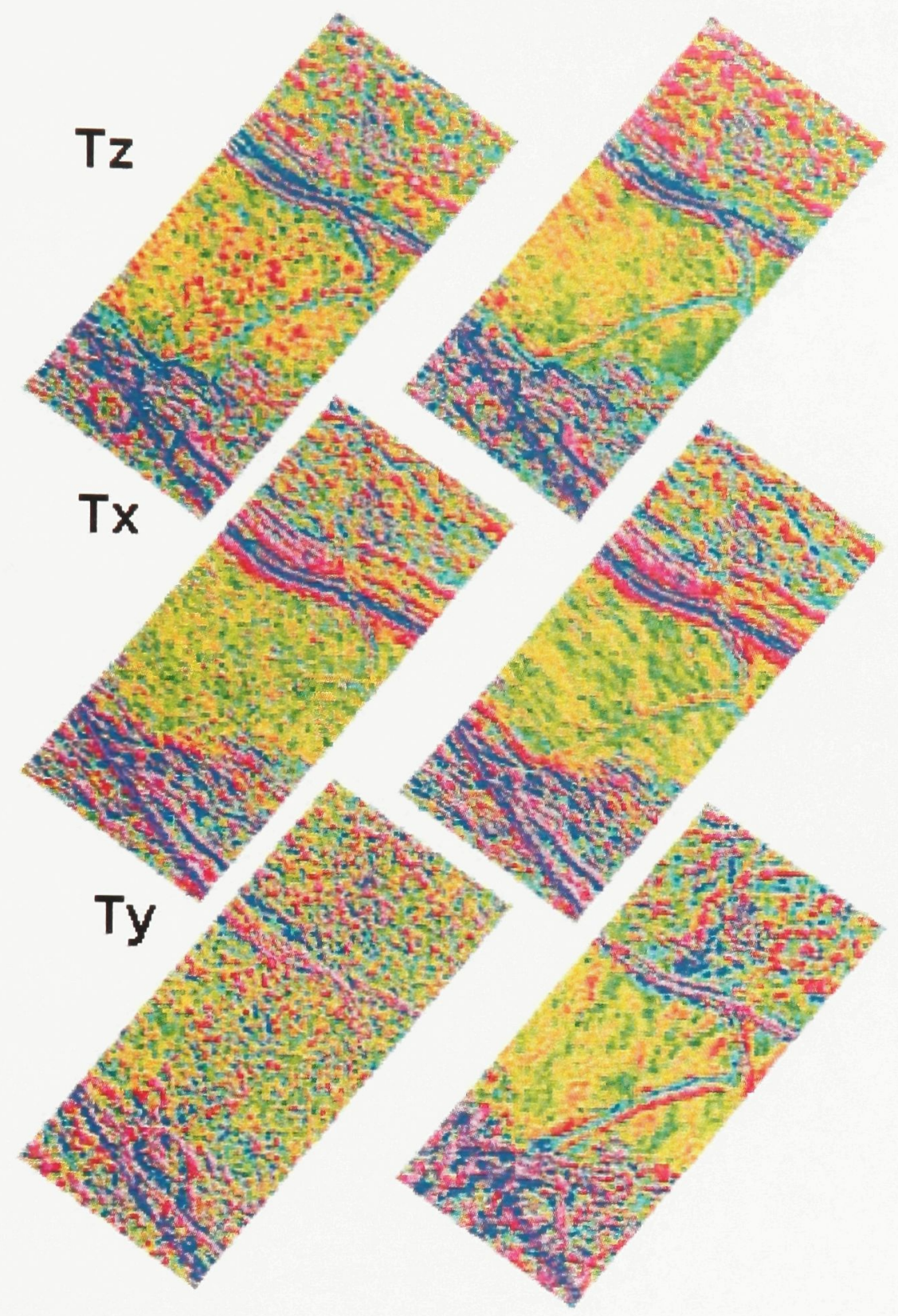

Figure 2.3

Fugro Airborn Surveys Heli-Triax system survey results. Figure shows a comparison between measured gradients, on the left, and calculated gradients, on the right. $T z$ is the vertical gradient, $T x$ the longitudinal gradient, and $T y$ the transverse gradient. Figure taken from Mushayandebvu and Davies (2006). 
with the lowest level of quality. Sources of noise are thought to be due to changes in orientation of the Heli-Triax system during flight. The extra resolution that was thought to be gained from measuring the magnetic gradient did not prove to be fruitful due to the higher level of noise and lower capability to trace magnetic trends.

\subsection{Unmanned Aircraft Systems (UAS)}

\subsubsection{UAS in Military and Civilian Applications}

The development of UAS was conducted predominantly with military applications in mind. The first UAS was developed by Samuel P. Langley and flown in 1896 for the purpose of flight research (Schwing 2007). The next application of UAS was by the American military in 1928. They developed an armed UAS but the project was subsequently cancelled due a lack of funding. UAS development continued with their application as target drones for pilot and gunnery training (Schwing 2007). In 1962, the application of UAS was expanded once again with the roles of surveillance, reconnaissance, and propaganda leaflet dropping missions. Since then, the military application of UAS has been sporadic in most countries except Israel who continued and later expanded their use. In 1989, the American Department of Defense established an Unmanned Aircraft Systems Roadmap that led to an increase in the development and application of military use UAS into the areas of strategic intelligence and tactical strikes (Office of the Secretary of Defense 2005, Schwing 2007). The US Roadmap acknowledges that one of the primary benefits of using UAS is the elimination of risk to aircraft crew flying hazardous missions (Office of the Secretary of Defense 2005). The 
recent successful application of UAS by the American military in the war in Iraq and Afghanistan has led to the creation of military use UAS development programs in several countries around the world.

There are currently several prominent civilian use UAS development programs around the world, some of these programs have created UAS such as the Helios, the Aerosonde, the Raven, and the Ikhana. The Helios is a high-altitude UAS built by AeroVironment Inc. for NASA's Environmental Research Aircraft and Sensor Technology Project. In 2001, the Helios beat the world record for sustained horizontal flight by a winged aircraft by reaching an altitude of 29,523 m above mean sea level. The project was designed to demonstrate the capability of UAS for high altitude and long duration flight, as well as the usefulness of UAS to scientific and civil applications (Noll et al. 2004). The Aerosonde series of UAS, developed starting in 1992, are designed for meteorological surveying in stormy weather conditions. The Aerosonde has been noted as the first UAS to cross the North Atlantic Ocean and had been employed in both meteorological and climatological surveying (Holland et al. 1992, Holland et al. 2001). The Raven UAS was developed by the University of Bologna for the purpose of high resolution measurement of atmospheric gasses within or near active volcanoes. The aim of the project is to provide advanced warning to mitigate damage from volcanic natural hazards (Saggiani et al. 2007). The Ikhana unmanned science and research aircraft system is actually a modified military Predator B UAS. It was modified by the Dryden Flight Research Center at NASA for the purpose of Earth Science research missions and advanced aeronautical technology development. It was also used successfully in 2006 and 
2007 to monitor forests fires that raged in southern California (Ambrosia et al. 2007, Allen and Walsh 2008).

After the March $11^{\text {th }} 2011$ magnitude 8.9 Tōhoku earthquake and tsunami in Japan, a UAS was used to monitor the radiation levels leaking from the damaged Fukushima Daiichi nuclear power plant (Smith 2011, Honeywell 2011). Built by the American company Honeywell, the T-Hawk is a military reconnaissance UAS that has been equipped to carry imaging sensors and a radiation sensor (Honeywell 2011). With its small-size and its vertical take-off, landing, and hovering capability, the T-Hawk is uniquely suited to fit into and image small cracks in reactor housing (Honeywell 2011). The capability of the radiation sensors provides information as to the intensity of the radiation contamination. This is important information that is required in order to make the decision to send in engineers to effect repairs on the damaged reactor. The successful use of the T-Hawk provided quick and reliable information that would have otherwise been gathered by sending an engineer into a hazardous situation (Warry 2011).

\subsubsection{Key enabling UAS technologies}

Recent technological advances within the fields of aircraft composite materials, electronics, computers, and in GPS and RADAR navigation have provided an opportunity to extend the capabilities of UAS towards uses that require a flight performance that has only conventionally been achieved by the most daring pilots. In particular, the advent of GPS, differential GPS, high-accuracy RADAR, and laser 
altimeters have enabled high accuracy flying. These technologies when applied to UAS extend the capability of flying to near surface altitudes as UAS are capable of higher manoeuvrability over conventional aircraft due to their smaller size and low weight (Macnae 1995).

The nature of the composite materials that are available not only allow the creation of an ultra light aircraft that requires little fuel, but it also creates the opportunity to perform aeromagnetic surveying using an aircraft with a smaller magnetic signature compared to manned aircraft. Recent advances in electronics have led to the creation of smaller yet more powerful computers and components, such as servos, that are increasingly more powerful while being more economical in their use of power. These improvements have led to the creation of Object Detection and Avoidance (ODAV) systems that had previously been unfeasible due to their high computational and power requirements. ODAV systems represent a critical component for all UAS, especially those designed for low altitude surveying. ODAV systems are required in low altitude flights in order to detect and avoid both moving and stationary objects that may be in or moving towards the flight path of the UAS. Proven ODAV systems that are robust and error free are also a mandatory requirement by transportation authorities to approve the use of UAS in civilian airspace (ASUAPO AIR-160 2008). 


\subsubsection{Unmanned Aeromagnetic Surveying}

The intention to use UAS for aeromagnetic surveying was first put forward in 1992 as the objective of a project ran by an association of mineral companies, known as Amira International Ltd.. The UAS, codenamed the GUAVA, was a modification of the Aerosonde design that substituted atmospheric research sensors for a magnetometer. The GUAVA was thought to be economically feasible with the additional benefits of providing: the option of higher resolution data by surveying at lower altitudes, more precise data by being able to achieve a more accurate drape over the terrain, 24 hour all weather surveying capability, the capability to survey over hazardous terrain, and a significantly cheaper alternative to current aeromagnetic surveys conducted using manned aircraft (Macnae 1995). The results of the project were never published.

In 1996, the American company Geophex developed a remote controlled unmanned survey system, named GAUSS, designed to detect ordnance using microgeophysical surveying. Based on a model helicopter platform, this survey system is designed to detect and locate tiny magnetic anomalies within a specific area that is easily visible by the controller. The system has the capability to carry either a magnetometer or a small electromagnetic sensor, and sends the data wirelessly to a nearby computer base station that processes the data automatically and generates a map (Keiswetterg et al. 1996). A final report on the project released by Geophex in 1996 reported that the system proved to be capable at surveying but required additional development in aircraft stability (Geophex 1996). 
The use of UAS in commercial aeromagnetic surveying was not available until Fugro Airborne Surveys of Ottawa, Ontario, conducted a few surveys with their GeoRanger UAS in 2005. Developed starting in 2003 by Fugro Airborne Surveys in cooperation with the Insitu Group Inc. (Anderson and Pita 2005), the GeoRanger is a multi-use reconnaissance UAS that was modified from the Insitu SeaScan Naval UAS for aeromagnetic surveying for commercial exploration of natural resources. It has the capability to record the total intensity of Earth's magnetic field using a single custom Scintrex CS-3 cesium vapour magnetometer stored in the fuselage. This magnetometer has a sensitivity of $0.01 \mathrm{nT}$ and is coupled with a less sensitive Billingsley TFM100 3Axis fluxgate magnetometer that provides data necessary for post-survey magnetic compensation of aircraft noise. The GeoRanger is built from magnetically inert graphite composites and fibreglass, and has a wingspan of $3 \mathrm{~m}$ with an endurance of 15 hours at a survey cruising speed of $25 \mathrm{~m} / \mathrm{s}$ ( 49 knots). It is engineered to be durable and to be broken into components to facilitate transportation and repairs. For surveying, it is engineered to be launched from a catapult and retrieved by a wingtip hooking system which eliminates the requirement of having a runway for operations, and provides the capability of operation at sea or in remote areas. The GeoRanger I uses a regular radio transmitter for receiving commands while remaining within sight of the controller. The GeoRanger II is equipped with an iridium satellite data transmitter for beyond line of sight operational capability. Although the results of initial surveying proved to be successful, restrictive UAS flight regulations prompted the company to temporarily suspend development of, and services offered by, the GeoRanger. 
Another UAS engineered for commercial aeromagnetic surveying was designed starting in 2004 by Universal Wing of Vancouver, British Columbia. The Venturer UAS is built with non-magnetic carbon composite and fibreglass materials. It has a $4 \mathrm{~m}$ wingspan and is configured as a horizontal gradiometer with a cesium magnetometer on each wingtip. A fluxgate magnetometer is located within the fuselage to provide magnetic compensation for aircraft noise. The system is run using a ground station that has the capability to coordinate surveying using up to 5 UAS simultaneously. Universal Wing has conducted and offers commercial aeromagnetic surveying using the Venturer within Canada and other regions.

Over the last several years UAS technology has been applied towards aeromagnetic surveying on a commercial basis. Although the service is available, there have been no studies reported in the scientific and engineering literature that explore aeromagnetic surveying using UAS or the advantages of the UAS versus manned aircraft. In addition, no studies were conducted that detail the most effective operational use of UAS for aeromagnetic surveying. Such questions are important to determine the practicality of aeromagnetic surveying using UAS and to determine optimal surveying methods.

\subsubsection{Regulatory Framework for Unmanned Aircraft Systems}

Unmanned aircraft system (UAS) is defined by Transport Canada within the Canadian Aviation Regulations as a powered aircraft that is not for recreational use and is 
operated without a flight crew member on board (Transport Canada 2010). There is no standardized classification of the different types of UAS, however they can be categorized by payload, as adopted by the US Military (Allen and Walsh 2008), or by weight, as adopted by Transport Canada and the European Aviation Safety Agency. Changes to flight regulations, as outlined by the Civil Aviation Working Group report completed by Transport Canada in 2007, recommends the categorization of UAS into three weight classes; less than $35 \mathrm{~kg}, 35 \mathrm{~kg}$ to $150 \mathrm{~kg}$, and $150 \mathrm{~kg}$ or more (Transport Canada 2007). The weight of the GeoSurv II with all of the electronics and fuel is expected to exceed $150 \mathrm{~kg}$.

There are strict regulations placed on the use of UAS by regulatory agencies. In both Canada and the United States, UAS are not permitted access to the Canadian Airspace, or the National Airspace System without flight specific approval from officials (Transport Canada 2010, ASUAPO AIR-160 2008). Current legislation does not allow the UAS operators to file (a flight plan) and fly (the mission). Instead, special permits are required such as Special Flight Operations Certificates (SFOC) in Canada. The main reason is safety concerns that will be alleviated only once reliable ODAV systems are developed and integrated onboard UAS. Another recommendation by the Civil Aviation Working Group is to limit the requirement of obtaining a SFOC to situations where the UAS weight is more than $35 \mathrm{~kg}$ and where flights exceed visual range (Transport Canada 2007). 


\section{Survey Site}

To determine the contribution low altitude flight provides towards the delineation of geological structures and to evaluate the aeromagnetic data acquisition capability of the sUAS, thereby predicting the capability of the UAS, a survey site was chosen with specific requirements. The requirements include a complex geological structure that contains smaller structures with fine detail. The detail of these small structures will become increasingly better delineated when measurements are made with increasing resolution. In addition, the survey site is required to contain geological units with strong and weak magnetic susceptibility, and have few sources of cultural magnetic noise. The site must have also been surveyed previously with a fixed-wing aircraft for comparison purposes. The survey site selected is shown in Figure 3.1. The site is an $8.5 \mathrm{~km}^{2}$ area in the Mazinaw terrain of the Central Metasedimentary Belt of the Grenville Province, and is situated near the town of Plevna in Ontario.

In 1983, the Ontario Geological Survey (OGS) commissioned Kenting Earth Sciences to conduct an aeromagnetic survey of the test survey site. It was flown by a Piper Navajo aircraft equipped with two Varian Associates cesium optical resonance magnetometers which were mounted at the end of a stinger with a vertical gradiometer separation of $1.83 \mathrm{~m}$. The survey was conducted at a fixed altitude of $150 \mathrm{~m}$, and along NW to SE flight lines which are separated by $200 \mathrm{~m}$ from each other. The survey data was compensated for magnetic interference created by the aircraft, was corrected for lag, diurnal variations, and IGRF, and underwent levelling and then micro-levelling. The 


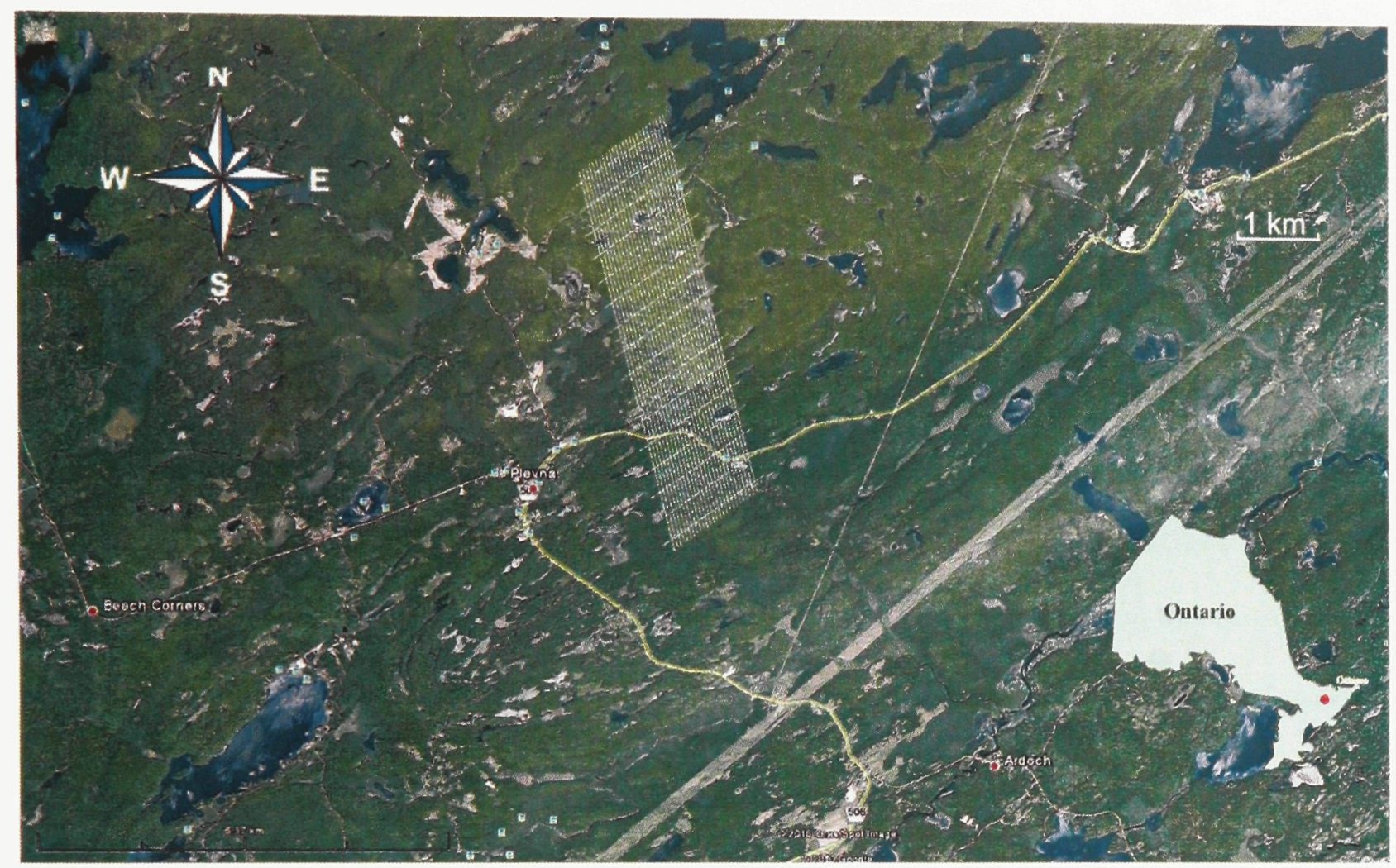

\section{Figure 3.1}

Google Earth satellite image of the survey site. The survey site is located in south-eastern Ontario near a town called Plevna. The survey block, shown with the white traverse lines running SE to NW and the control lines running SW to NE, has an area of about $8.5 \mathrm{~km}^{2}$. The different shades of green in the image are due to changes in tree type, from deciduous to coniferous, and changes from forests to bogs. The changes in relief are an indication of the rugged nature of the terrain. 
results of this survey include a TMI and a vertical gradient map (Ontario Geological Survey 2003).

Information on the geology of the site was gained from two OGS publications completed in 2006 by R.M. Easton. The first publication listed (Easton 2006a) contains geologic information containing rock descriptions and an interpretation of the structural geology. The second publication (Easton 2006b) is a structural geology map of the region. Figure 3.2 is a simplified structural geology map, modified from Easton 2006b. The modifications were to the colour used to identify rock units to make them more easily distinguishable, and to remove strike and dip measurements as well as mineralization markers and isogradient lines. Both of the OGS data sets, along with published maps, are available free of charge from the OGS to the general public.

The structural geology of the survey site shown in Figure 3.2, is complex. The fold shown is an interference pattern created by two separate generations of folding due to tectonic events (Easton 2006a). The true nature of this fold is unknown; it has the visual characteristics of a vertical isoclinal similar fold, but the rocks units indicate that it is an upright tight or horizontal isoclinal fold. The portion of the fold surveyed is known as the Plevna Antiform, and is the northern counterpart of the Plevna Synform to the south (Easton 2006a). The fold is composed of metasedimentary rocks inter-bedded with strongly magnetic magnetite bearing metavolcanic rocks. These two rock types are juxtaposed against weakly magnetic carbonate metasedimentary rocks. All of the rock 


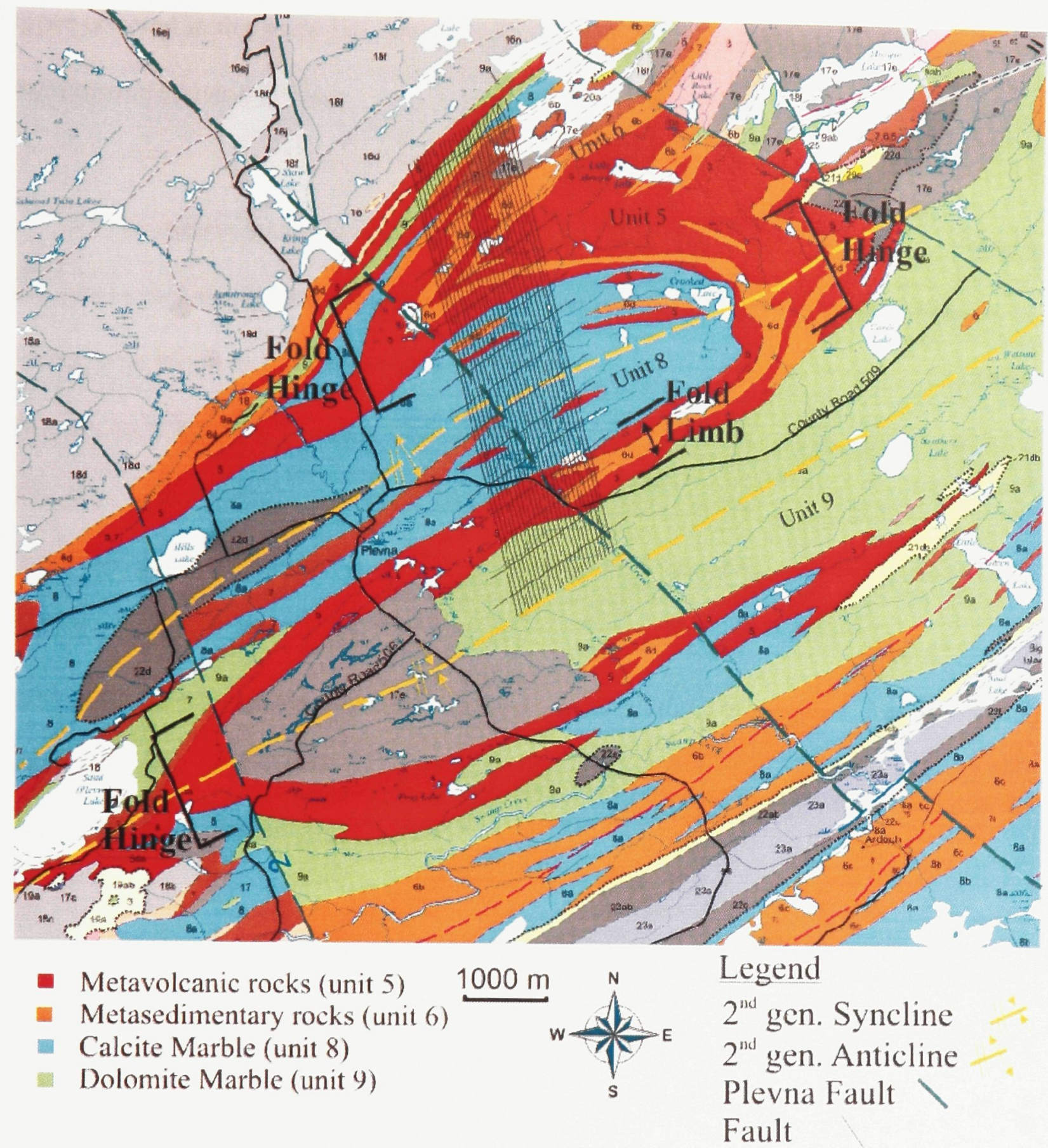

\section{Figure 3.2}

Structural geology map of the region around the survey site. The survey site is marked by the superimposed sUAS traverse and control flight lines, shown in black. The red represents the location of magnetically strong metavolcanic rocks with magnetite porphyroblasts. The orange represents the location of volcanic metasedimentary rocks; their magnetic susceptibility is unknown. The blue and green are magnetically weak calcite and dolomite marbles, respectively. After Easton (2006b). 
types covered in the study site belong to the Mazinaw domain within the Grenville Supergroup (Easton 2006a).

The metavolcanic rocks, shown on Figure 3.2 in red and marked unit 5, belong to the Mazinaw Lake Formation and are about 1245 million years old. The formation is volcanic in origin and contains metamorphosed fine grained basaltic and rhyolitic rocks that are both tholeiitic in composition. There are four types of rocks in this formation. The first type is a finely laminated pink coloured garnet-plagioclase-magnetitequartzofeldspathic gneiss. The second type is a foliated garnet-magnetite-hornblendebiotite-plagioclase gneiss. The third rock is a finely laminated pink coloured quartzofeldspathic gneiss which it thought to have formed from a pyroclastic deposit. The fourth rock is a finely laminated dark green coloured biotite-hornblende-plagioclase gneiss (Easton 2006a).

The metasedimentary rocks that are coloured orange in Figure 3.2, and marked with unit 6 are interpreted to be metamorphosed volcanic sediments. The rocks are black coloured amphibolites, and can be separated into 3 types. The first is a biotitehornblende-quartz-plagioclase gneiss, the second a hornblende-plagioclase gneiss, and the third a quartzofeldspathic gneiss. Many of these rocks were found to have garnet porphyroblasts. The magnetic susceptibility of these rocks are unknown and this unit is undated (Easton 2006a). 
The calcite and dolomite marble, unit 8 and unit 9, and coloured light blue and light green, respectively, are both coarse grained and massive. The calcite marble may include some metavolcanic rocks from unit 5 and some metasedimentary rocks from unit 6d. The dolomite marble may include zones of calc-silicate rocks. Marble is typically inert in terms of magnetic susceptibility. These units are undated (Easton 2006a).

Within the north-eastern portion of the survey site, a clastic metasedimentary rock type (unit 7) is inferred. This rock type is thought to be a mix of volcaniclastic and siliclastic rocks that were each deposited in turn, with the origin being the Plevna volcanic centre. The rock facies changes with increasing distance from the volcanic centre (Easton 2006a). The magnetic susceptibility of this rock is unknown, and the location of this rock unit in figure 3.2 is inferred. This rock unit is not well surveyed by either the sUAS or the ground survey as it falls in the north-eastern extent of the survey area.

The Plevna fault is a major structural feature that is inferred on the structural geology map in Figure 3.2. The fault has a strike that varies between azimuths of $290^{\circ}$ to $320^{\circ}$ degrees (Black and Rencz 1987). It is known to follow the same strike as the Ottawa-Bonnechere graben, and was found to have occurred at about 599 Ma using hydrothermally deposited rutile along with uranium-lead dating (AECB Workshop 1995, Easton 2006a). The Plevna fault is known to have a vertical displacement as high as $40 \mathrm{~m}$ in certain areas, and a lateral displacement near Crotch Lake of about $300 \mathrm{~m}$ (Pauk 1982). 
Other areas along the fault show little or no displacement at all. In terms of magnetic susceptibility the fault is known to be invisible or weakly magnetic (Easton 1995). The fault has been detected in some areas when measuring the vertical gradient of the magnetic field (Easton 2006a).

The terrain in the survey site is rugged, as illustrated in Figure 3.3 which plots the distribution of changes in elevation gradient. The site is covered by a forest with a combination of both deciduous and coniferous trees that have a maximum height of about $25 \mathrm{~m}$. There are also several small lakes, streams, and bogs of various sizes. Outcrops and glacial erratics are common in occurrence throughout the site. The northern end of the site has a few seasonal cottages that are powered by a standard power line that runs along Grindstone Lake Road. The southern end of the site crosses County Road 509, which also has a standard power line running along it. 


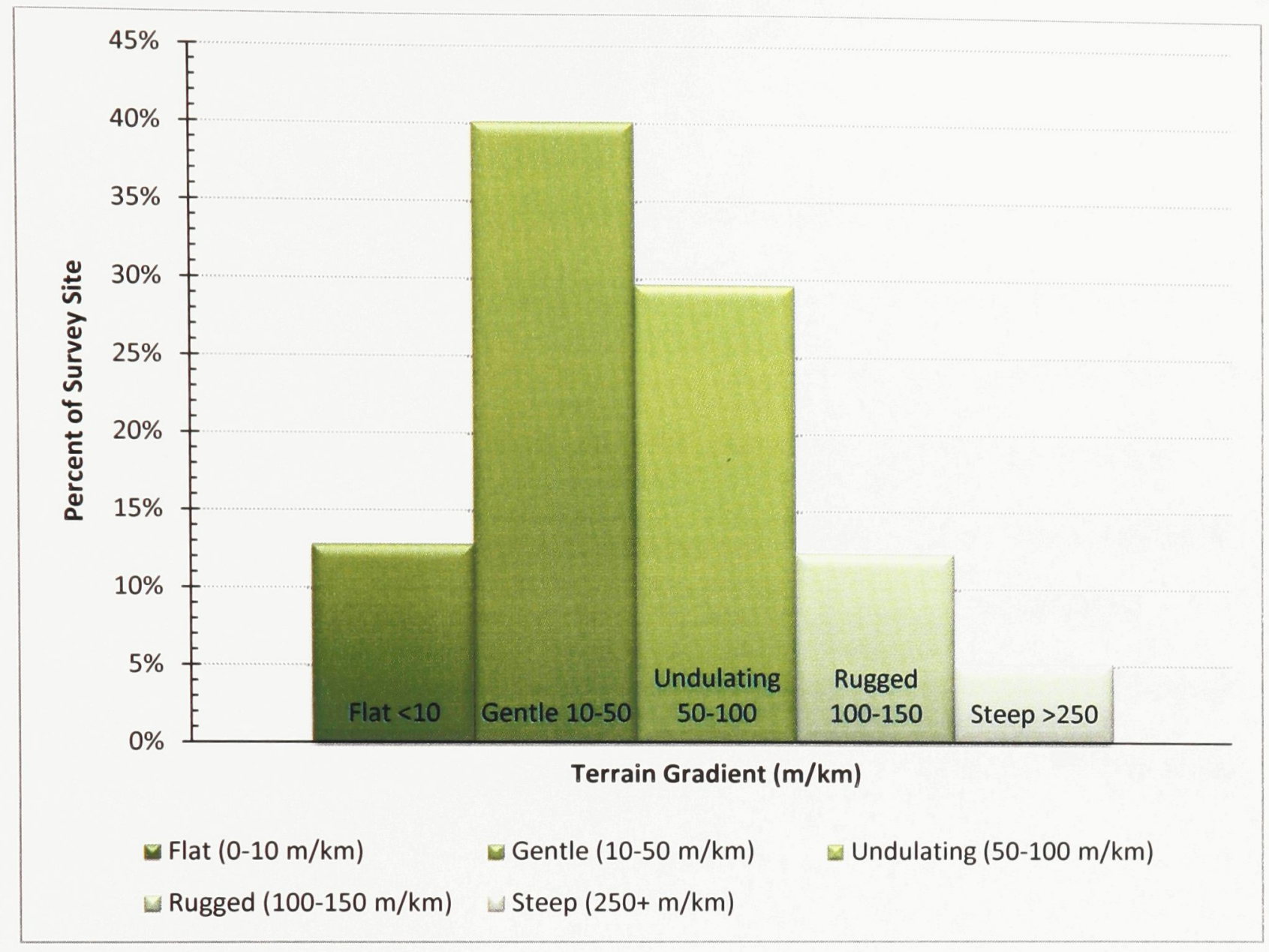

\section{Figure 3.3}

Distribution of terrain gradients within the survey site. Terrain gradient data is calculated from SRTM data. 


\section{Data Acquisition and Processing}

\subsection{1 sUAS Survey}

Two surveys were carried out within the survey site as part of this project. The first survey (February 2010) was done using the sUAS to provide a data set acquired at an altitude typical to what will be adopted by the UAS when it becomes available. The purpose of the sUAS survey is to make measurements that capture the same range in frequencies and amplitudes that would have been made by the UAS. Another purpose is to evaluate the capability of the gradiometer configuration of $4.67 \mathrm{~m}$ to record the gradient of the magnetic field. The second survey (February and November 2010) aimed at measuring the magnetic field at the highest resolution that is attainable, which is along the ground.

\subsubsection{Survey Planning}

Survey planning begins with computer modeling. Since the sUAS will be suspended beneath a helicopter by a $33 \mathrm{~m}$ cable and that the desired sensor altitude is 50 $\mathrm{m}$, the helicopter will be flying at an altitude of $83 \mathrm{~m}$ above ground level. As this is considered a low altitude flight, flight hazards with respect to terrain and structures must be considered. The terrain is analyzed by using radar altimeter data gathered by the Shuttle Radar Telemetry Mission (SRTM). The SRTM map, shown in Figure 4.1, is processed and combined with survey parameters in order to create a drape. The drape is 


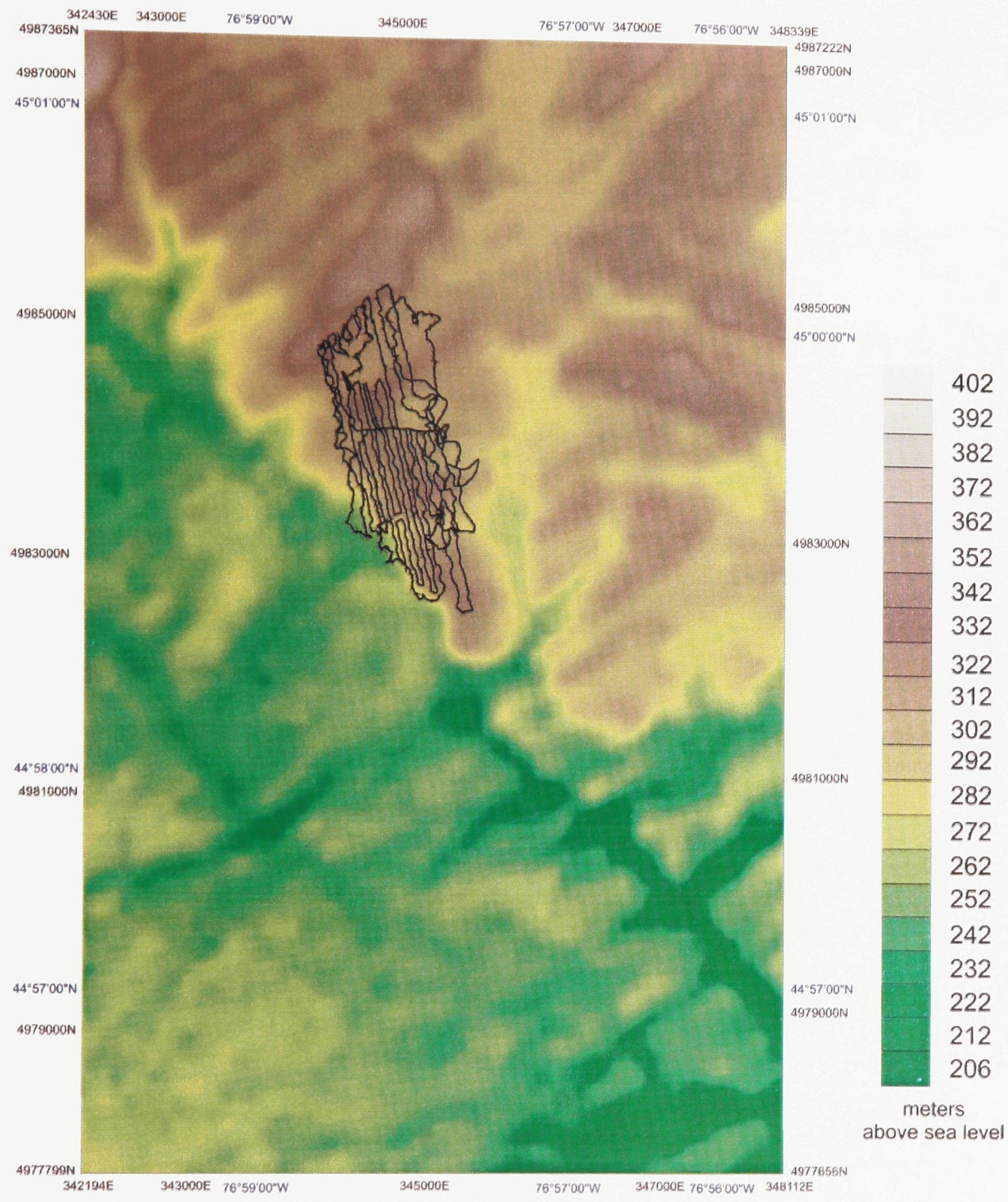

\section{Figure 4.1}

SRTM map of the survey site. This digital terrain model has a cell size of $100 \mathrm{~m}$. The path covered by the ground survey is shown in black. 
designed to ensure the helicopter maintains a set altitude above ground throughout the survey. The helicopter will descend in valleys, and ascend over hills in order to maintain that set altitude above ground. This is done to create an unbiased dataset where the magnetic intensity measured over a hill is comparable to that measured over a valley (Reeves 2005).

In the case of the sUAS, the helicopter was flying a drape that smoothed the topography of the terrain to a variation that did not exceed $57.6 \mathrm{~m}$ of change in altitude per horizontal kilometre. The median altitude of the drape with respect to the sUAS is configured to be $50 \mathrm{~m}$ with the lowest possible altitude at no less than $33 \mathrm{~m}$. There was no upper limit configured for the altitude of the aircraft. This means the sUAS could be at a lower altitude or at a higher altitude with respect to $50 \mathrm{~m}$ throughout the survey site. In areas where the sUAS was measuring the magnetic field at an altitude less than $50 \mathrm{~m}$ above ground, the magnetic field would be recorded at a higher intensity than what would have been recorded had the sUAS been at $50 \mathrm{~m}$. Similarly if the sUAS recorded the magnetic field while it was at an altitude that was greater than $50 \mathrm{~m}$, the intensity of the recorded value would be less than the value that would have been recorded at $50 \mathrm{~m}$ altitude. Since the main purpose of the drape is a smoothing of the topography, the sUAS is likely to be flying below an altitude of $50 \mathrm{~m}$ when travelling over a steep hill, and above an altitude of $50 \mathrm{~m}$ when flying over a valley situated between hills. The magnetic intensity is then recorded at a higher resolution over hills, and a lower resolution over valleys. In comparison, the ground survey was conducted at a set distance above ground that did not vary throughout the survey site, as the magnetometer was being carried by a 
person, in a standard backpack. The recorded magnetic intensity is recorded at the same resolution throughout the area covered. This concept is illustrated in Figure 4.2.

Within the survey site, a grid is designed so that the primary surveying lines, called traverse lines, are oriented perpendicular to the main geological structure. For this survey site, the main geological structure is the fold. It is the traverse lines that will provide the most detailed observations about the fold. Lines that are flown perpendicular to the traverse lines, termed control lines, are flown at a larger separation and are used to correct the recorded magnetic intensities along each line to a comparable level. The intersection of the control and traverse lines provide a point in space where the magnetic field has been measured twice. These successive points of measurement therefore provide a reference with which to correct, or level, the traverse lines (Reeves 2005). Unlevelled lines result in spurious differences in measured magnetic field intensity due to changes in altitude or diurnal variations between measurements. Once the survey grid has been finalized, it is merged with the flight drape.

Before an aeromagnetic survey is carried out, corrections are typically made to attenuate magnetic interference that is introduced into the data by the magnetic fields generated by the operation of the aircraft. There are several sources of magnetic interference associated with the operations of an aircraft. The first is due to the permanent magnetization of the ferromagnetic materials that make up a plane. The magnetic field, that is associated with these materials, changes in shape and intensity with different aircraft headings, and will interfere with the measurement of Earth's magnetic field 


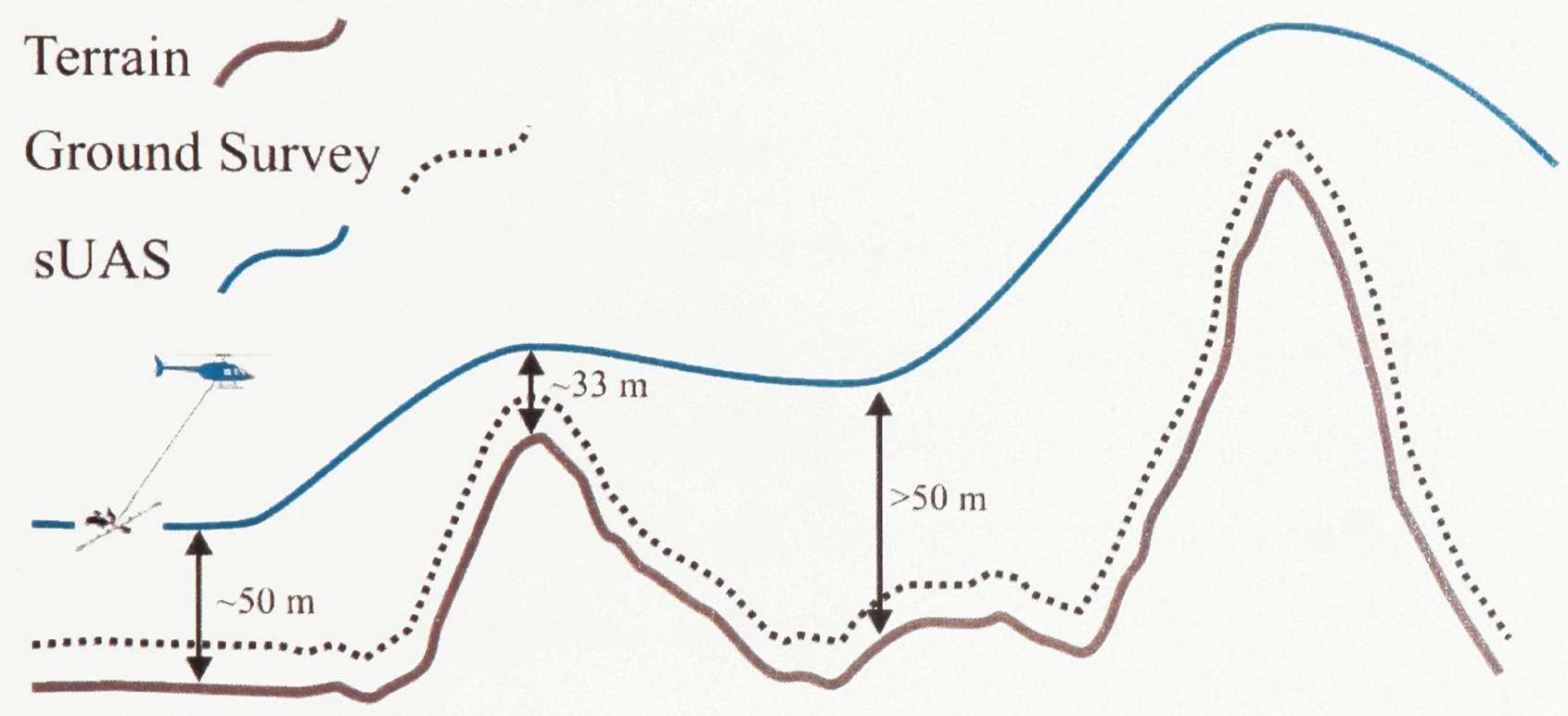

Figure 4.2

Terrain clearance comparison between the sUAS drape and the ground survey. 
(Leliak 1961). The second type of magnetic interference is due to the induction of a magnetic field in ferromagnetic materials by Earth's magnetic field. These induced fields do not change in direction with the aircraft, but will change in direction and intensity with respect to Earth's magnetic field (Leliak 1961). The error caused by the first and second types of magnetic interference are dependent upon the heading of the aircraft and is termed heading error. The third type of magnetic interference is due to fields induced by varying electromagnetic fields that are generated from the aircrafts electrical components. They can also be generated by eddy currents within Earth's magnetic field that act upon the aircraft during flight manoeuvres (Leliak 1961). These induced fields change in size and direction as the aircraft undergoes changes in attitude in the pitch, roll, and yaw directions, and is termed manoeuvring noise. The heading error and manoeuvring noise are corrected by a compensation test and system first described by Paul Leliak in 1961 .

A compensation test is performed by first measuring the effect that the heading error and manoeuvring noise has on the magnetic field recorded by the magnetometers. This is done by flying a series of manoeuvres at an altitude where Earth's magnetic field is fairly homogeneous in the horizontal plane so that changes in the magnetic field due to the respective changes in pitch, roll, and yaw of the aircraft can be isolated. The aircraft flies a fixed altitude flight path in the shape of a square. Along each side of the square the aircraft executes $\pm 5^{\circ}$ pitch, $\pm 10^{\circ}$ roll, and $\pm 5^{\circ}$ yaw manoeuvres (Reeves 2005). Each manoeuvre is conducted independently and only for a few seconds. Compensation coefficients, first developed by Hardwick in 1984, designed to nullify the field variations due to manoeuvring are then applied to the data. The results are tested by flying the 
figure of merit test. The figure of merit test is a repeat of the compensation manoeuvres using the applied compensation coefficients. The results of the tested are determined by the 12 terms that make up the figure of merit. The terms are calculated by adding the differences in field measurements between extents measured during each of the pitch, roll, and yaw manoeuvres for each cardinal direction. A figure of merit for an acceptable compensation is a fraction of a nanoTesla (Reeves 2005). Another test to verify that the heading error has been corrected is called the clover leaf test. In this test, the aircraft flies over a sharp magnetic anomaly from each of the cardinal directions. The objective of the test is to verify that the anomaly is recorded at the same intensity regardless of the direction from which it was sampled (Reeves 2005).

Since the sUAS is non-magnetic and was suspended by a $33 \mathrm{~m}$ cable, which is outside of any significant sources of magnetic interference caused by the helicopter, and since the sUAS is composed of non-magnetic metals and composites, it is assumed that the sUAS did not experience a significant amount of interference due to the heading. Since the sUAS is designed for level and stable flight, it is assumed that the sUAS is not experiencing significant changes of pitch, roll, or yaw during surveying. As a result, it is also assumed that there was no significant interference introduced into the data as a result of manoeuvres made during flight by the helicopter, and therefore no compensation of the sUAS magnetic data set was required. The compensation system and tests will have to be performed by the UAS once it is developed and used for aeromagnetic surveying. 


\subsubsection{Survey Execution}

The sUAS survey was conducted on February $12^{\text {th }} 2010$ at a nominal altitude of $50 \mathrm{~m}$ above ground level. The flight plan followed a gradient that did not exceed a change in altitude over $57.6 \mathrm{~m} / \mathrm{km}(350 \mathrm{ft} / \mathrm{NM})$. It was planned with a traverse line heading of $168^{\circ}$ (measured clockwise from north) with a line spacing of $50 \mathrm{~m}$, and with control lines that have an heading of $63^{\circ}$ with a $300 \mathrm{~m}$ line spacing. The survey plan was composed of 26 traverse lines and 16 control lines. Figure 4.3 shows the survey lines superimposed on an image of the flight drape. As an additional test, line 1010 was reflown at an altitude of $100 \mathrm{~m}, 150 \mathrm{~m}$, and $200 \mathrm{~m}$. While the sUAS survey was being conducted, the helicopter recorded the altitude of the aircraft above the ground using laser and radar altimeters. Figure 4.4 shows a photograph of the sUAS being flown during a test flight.

In order to apply diurnal corrections to the measured geomagnetic field, a ground station (GND) is required. For this purpose, a GPS enabled ground station was set-up at a private airstrip near Ardoch on Country Road 506. The location is $7 \mathrm{~km} \mathrm{~S} / \mathrm{W}$ from the survey site and is close enough to capture the diurnal variations measured by the sUAS during the survey.

The results of the sUAS survey include a TMI map, a FVD map, a calculated longitudinal gradient map, a calculated transverse gradient map, a measured transverse gradient map, and a digital terrain model (DTM) of the survey site. 


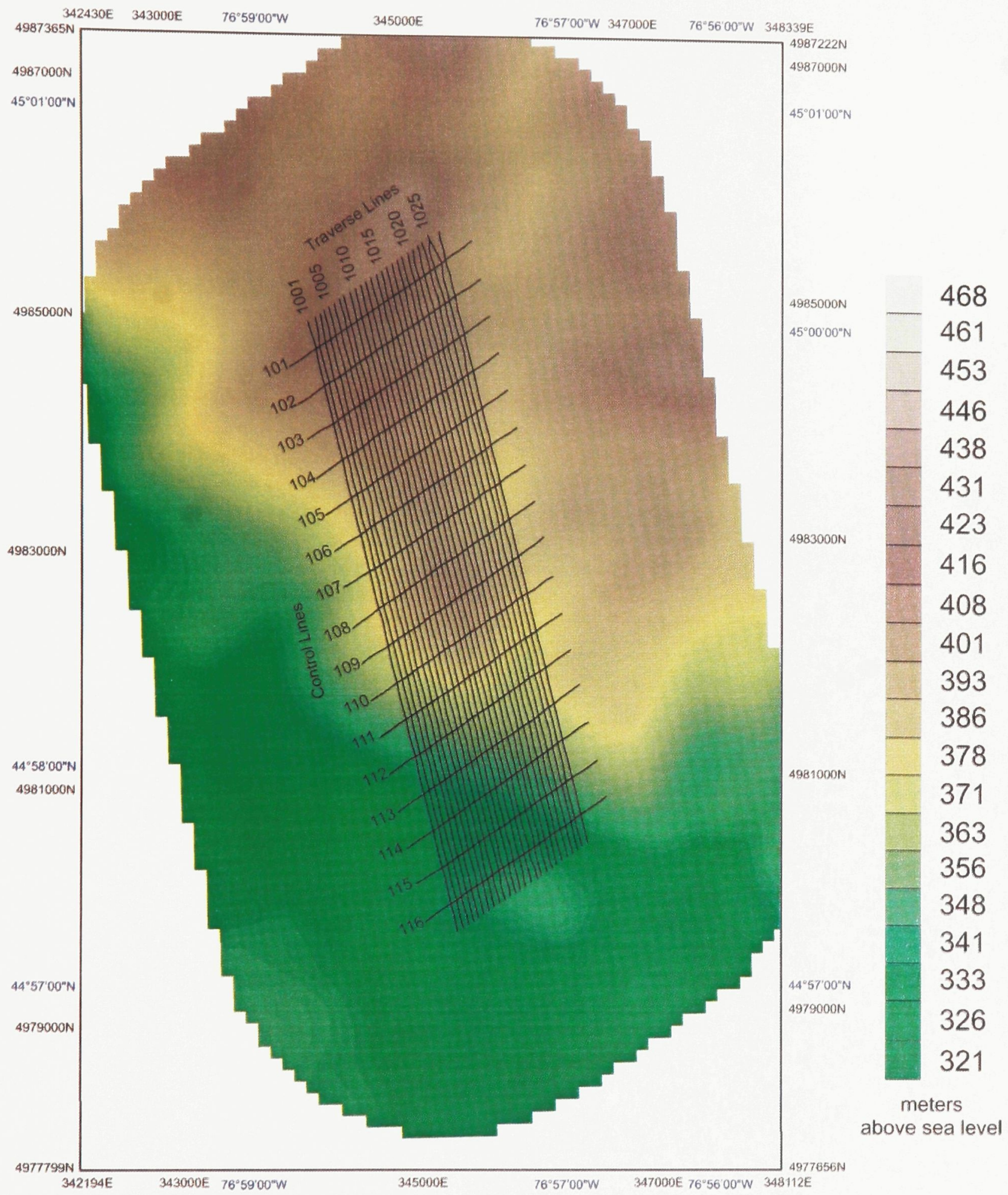

Figure 4.3

Pre-planned flight drape. The SRTM map in Figure 4.1 is smoothed in order to create flight paths that do not exceed an upward or downward gradient of $57.6 \mathrm{~m} / \mathrm{km}$. The sUAS survey lines are shown in black. Traverse line numbers run from 1001 to 1026 , and control line numbers run from 101 to 116. 


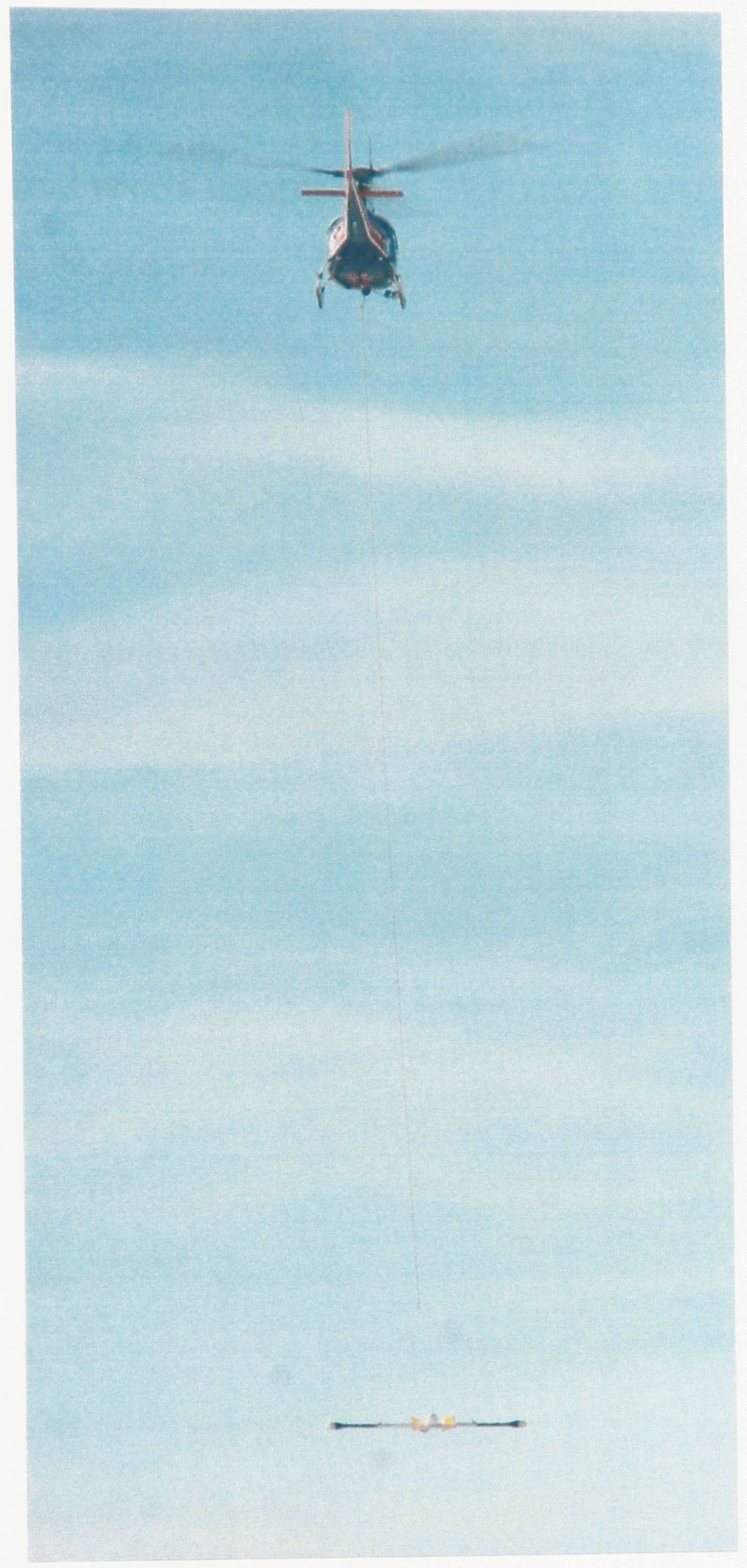

Figure 4.4

Picture of the sUAS being flown, suspended beneath a helicopter. 


\subsubsection{Data Processing}

A Sander Geophysics in-house data acquisition system was used to record the data gathered during the sUAS survey. The data acquired includes the data sets listed in

Table 4.1. The X, Y, and Z co-ordinates were recorded using the World Geodetic System 1984 revision (WGS-84).

A processing flowchart for the sUAS data is shown in Figure 4.5. The most important steps involve quality control, differential GPS (dGPS) correction, space and time lag correction, IGRF correction, diurnal correction, gradient calculations, and levelling.

The quality control step is standard across all data sets and includes checking the data for gaps, spikes, and basic integrity. Data gaps can be due to equipment failures or loss of signal. Spikes are indications of equipment problems or the presence of an external influence that is modifying the signal in an unusual way. A spike is usually created from the presence of a metallic object or a power line, and is considered cultural noise. Data from a confirmed source of cultural noise is corrected by having the spike removed from the data set. Few sources of cultural noise were detected during the sUAS survey. The final step with regard to quality control is to examine the data to verify if the individual values are within a reasonable range based on the area that was surveyed. All of the data sets acquired during the sUAS survey passed this test. 
Table 4.1: Table of Data Acquired During the sUAS Survey

\begin{tabular}{|l|l|}
\hline \multicolumn{2}{|c|}{$\begin{array}{c}\text { SUASder Geophysics Acquisition System } \\
\text { Data down-sampled to } 10 \mathrm{~Hz}\end{array}$} \\
\hline Port Cesium Mag & $0.001 \mathrm{nT}$ \\
\hline Starboard Cesium Mag & $0.001 \mathrm{nT}$ \\
\hline GPS Time & $0.01 \mathrm{~s}$ \\
\hline GPS X & WGS-84 at $0.01 \mathrm{~m}$ \\
\hline GPS Y & WGS-84 at $0.01 \mathrm{~m}$ \\
\hline GPS Z & WGS-84 at $0.01 \mathrm{~m}$ \\
\hline GPS Ephemeris Data & N/A \\
\hline Radar Altimeter & $0.1 \mathrm{~m}$ \\
\hline Laser Altimeter & $0.01 \mathrm{~m}$ \\
\hline \multicolumn{2}{|c|}{ sUAS Ground Station } \\
\hline Sander Geophysics Acquisition System \\
Sampled at 10 & $\mathrm{~Hz}$ \\
\hline GND Cesium Mag & $0.001 \mathrm{nT}$ \\
\hline GND GPS Time & $0.01 \mathrm{~s}$ \\
\hline GND GPS X & WGS-84 at $0.01 \mathrm{~m}$ \\
\hline GND GPS Y & WGS-84 at $0.01 \mathrm{~m}$ \\
\hline GND GPS Z & WGS-84 at $0.01 \mathrm{~m}$ \\
\hline GPS GND Ephemeris Data & N/A \\
\hline
\end{tabular}




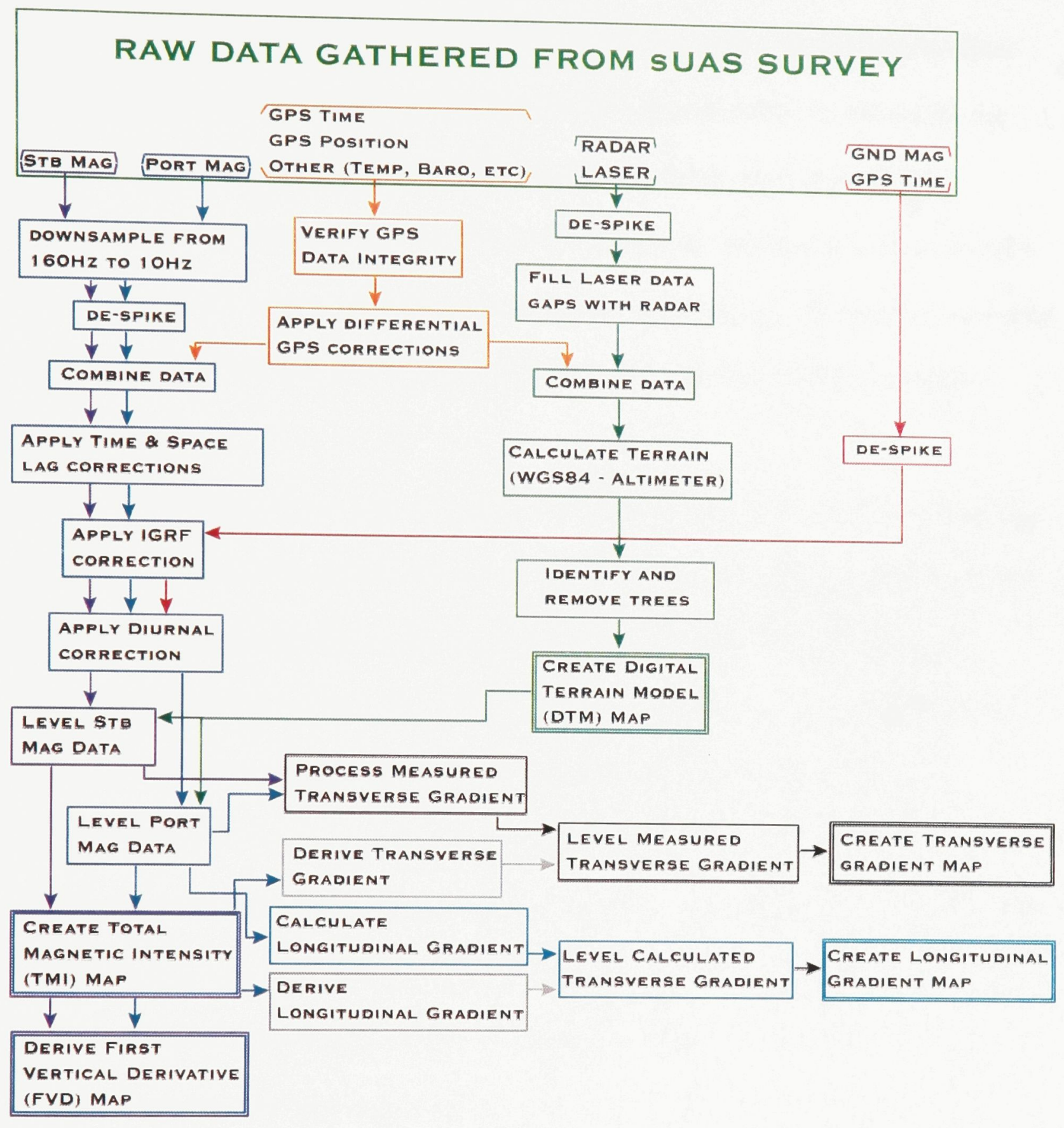

\section{Figure 4.5}

Flowchart showing the various steps performed in order to process the data that were gathered during the February 2010 sUAS survey. The processing resulted in the creation of 5 maps: a TMI map, a FVD map, a DTM map, a longitudinal gradient map, and a transverse gradient map. GND Mag represents data gathered at a ground station near the survey site. 
The following step is a differential GPS correction which uses ephemeris data, and data gathered from a nearby geo-referenced GPS ground station to correct for the timing errors that are introduced as a GPS signal travels from space through the atmosphere. The timing error can then be used to correct the positional error on the GPS data. For the sUAS survey, the GPS antenna on the helicopter was differentially corrected using the NCR1 Government of Canada dGPS reference station in Ottawa, Ontario.

An important step in magnetic data processing involves the correction of the data set for the lag effect. The lag effect has two components which are the space lag and the time lag. The space lag is due to the offset in space, in the horizontal transverse or longitudinal direction, between the GPS antenna and the location of the magnetometer. The space correction is a mathematical correction based on the average velocity calculated every $0.1 \mathrm{~s}$ with the purpose to move the magnetometer to be in line with the GPS so that both recordings coincide in space and time. The time lag is the time the data acquisition system takes to process and record the measurements, and is a property of the data acquisition system (Reeves 2005). For the sUAS survey, a technique was developed in order to estimate the space and time lags. This was done because the sUAS was suspended on a cable beneath the helicopter, and its exact location in time and space was unknown. In order to estimate the location of the sUAS, different lag corrections were applied to the data and then analysed using the root mean square (RMS) values of the difference in measurements of the magnetic field at the intersections between traverse and control lines. The RMS values are then grouped into bins based on their magnitude with the number of occurrences in each bin plotted against the RMS value of the bin, 
creating a distribution diagram. The distribution skewed towards the smallest bin values represents the optimal lag correction values. The final lag correction used for the sUAS is shown in Figure 4.6 by the red line. It corresponds to a time lag of $0.3 \mathrm{~s}$, and a space lag of $6 \mathrm{~m}$.

The IGRF correction is used to subtract, from the measured magnetic field, the portion of the Earth's magnetic field generated by the dynamo action within the outer core of the Earth (see section 2.2). The diurnal variations correction (see section 2.2) is used to remove the time-varying magnetic field from the measured magnetic field. Both of these corrections isolate changes in the magnetic field that are due to local and regional anomalies in or on Earth's crust.

The transverse gradient is calculated by subtracting the port magnetometer from the starboard magnetometer and dividing the result by the distance between the magnetometers (Hood and Tesky 1989). This is done with respect to cardinal direction to maintain data polarity. For the sUAS, the port magnetometer was subtracted by the starboard magnetometer with a northern heading, and vice versa with a southern heading. The result was then divided by $4.67 \mathrm{~m}$ which is the distance between the port and starboard magnetometers.

Levelling is a process by which the measured magnetic field that has been quality controlled and corrected for lag, IGRF, and diurnal variations, is corrected for altitude variations as well as residual diurnal variations. Altitude variations are deviations in 


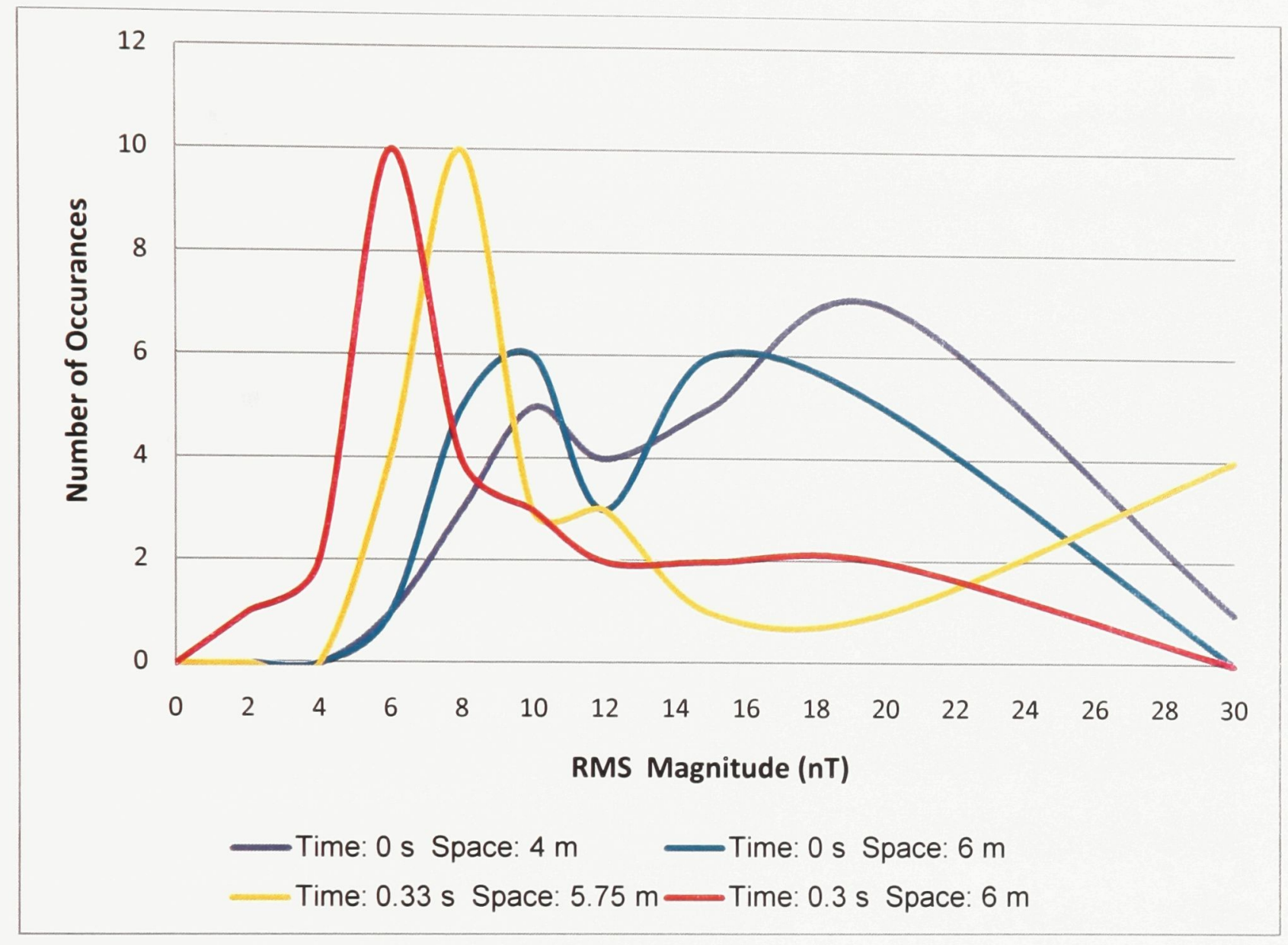

Figure 4.6

Comparison between four test configurations in order to determine the time and space lags of the sUAS with respect to the helicopter. The lag correction that results in the highest number of occurrences at the lowest magnitude RMS provides the best correction. It is plotted in red on the graph. 
altitude from the flight drape that contributes to a stronger or weaker magnetic intensity compared to the values measured along an adjacent flight line. The result is a linear ridge or valley in the mapped data set that has the same strike as the heading of the flight line. For a TMI map, both the altitude variation and the residual diurnal variations are corrected by shifting data from the traverse lines towards data from the control lines by a constant amount, in $\mathrm{nT}$, that is an average of the difference between the magnetic field measurements made at the intersections. An additional levelling step called fainting was also applied to the TMI sUAS map. Fainting is an interpretive manual correction of the magnetic field in order to remove a local linear anomaly that is identified by having the same strike as the line heading. The anomaly must be interpreted, by verifying the altitude of the aircraft and the DTM, to be caused by a diurnal variation or a deviation in surveying altitude. Fainting is also often used to correct for cultural anomalies. For TMI maps, the final step that was taken in this study, was to analyze the corresponding FVD grid for linear trends in the magnetic data. If a trend was discovered, an extra data point was inserted between the flight lines through which the trend passes, in order to constrain the interpolation.

The technique used to level the transverse gradient data is similar to that used to level the TMI data. Since the gradient is calculated in the transverse direction relative to the direction of flight, data from the control lines cannot be used for comparison without a gradient calculation conducted with respect to the same heading. To simplify levelling, and because a TMI map was already available, a transverse gradient was calculated from 
the TMI map. The calculated gradient was then used to level the measured gradient. The same technique was used once again to calculate and level the longitudinal gradient.

An optional step in data processing known as reduction to the pole (Reeves, 2005) was not applied to any of the TMI maps within this thesis. Reduction to the pole is a technique that is used to correct the shape of a magnetic anomaly as it becomes distorted due to the changes in inclination of Earth's Magnetic field with latitude. This correction was not applied because the survey area is relatively small and does not extend over several degrees of latitude.

\subsection{Ground Survey}

\subsubsection{Survey Planning}

As the ground survey is meant to provide the highest resolution total magnetic intensity map, it must have either the same line spacing or a smaller line spacing than the sUAS survey. As ground surveys are time consuming and expensive, the same traverse line heading of $168^{\circ}$ and line spacing of $50 \mathrm{~m}$ that was used to plan the sUAS survey was adopted for the ground survey. There were no control lines as the data did not need levelling. Due to the limited time available to perform the survey, the northern portion of the survey site, covering the fold hinge, was deemed to be of higher priority and was surveyed first. The southern portion of the survey site was not surveyed by ground due to time constraints. 
The GEM systems GSM-19 Overhauser walking magnetometer system was chosen to conduct this survey based on the following advantages. It is a proton precession type magnetometer, which does not require orientation into the magnetic field, and so it is easier to use than a cesium-vapour magnetometer. It is also lighter than cesium models, uses less power, and the model chosen came with a backpack system designed for use with ground surveys.

\subsubsection{Survey Execution}

The ground survey was completed in two separate field outings. Each field outing ran for a duration of 5 days. The first outing, shown in the photo in Figure 4.7, occurred in the last week of February 2010 and coincided with a major winter storm that saw a rise in temperature throughout the region from $-20^{\circ}$ to $0^{\circ}$ Celsius. The storm deposited two feet of snow along with freezing rain. The progress of the survey was slowed due to the deterioration of the weather conditions and the softening of the snow cover, which reduced mobility despite the use of snowshoes. As the results of the February 2010 survey did not cover a sufficient extent of the survey site required for the upwardcontinuation of the data-set, a follow-up November 2010 survey was planned. The November survey, shown in Figure 4.3, happened to coincide with a fall storm that saw freezing rain, and a small amount of snow deposited within the survey site. Progress was however sufficient to complete a significant portion of the survey area during the time allotted. Both the February and November 2010 surveys were conducted in cold weather, and close to or during winter, in order to ensure that the forest canopy had fallen, to avoid 


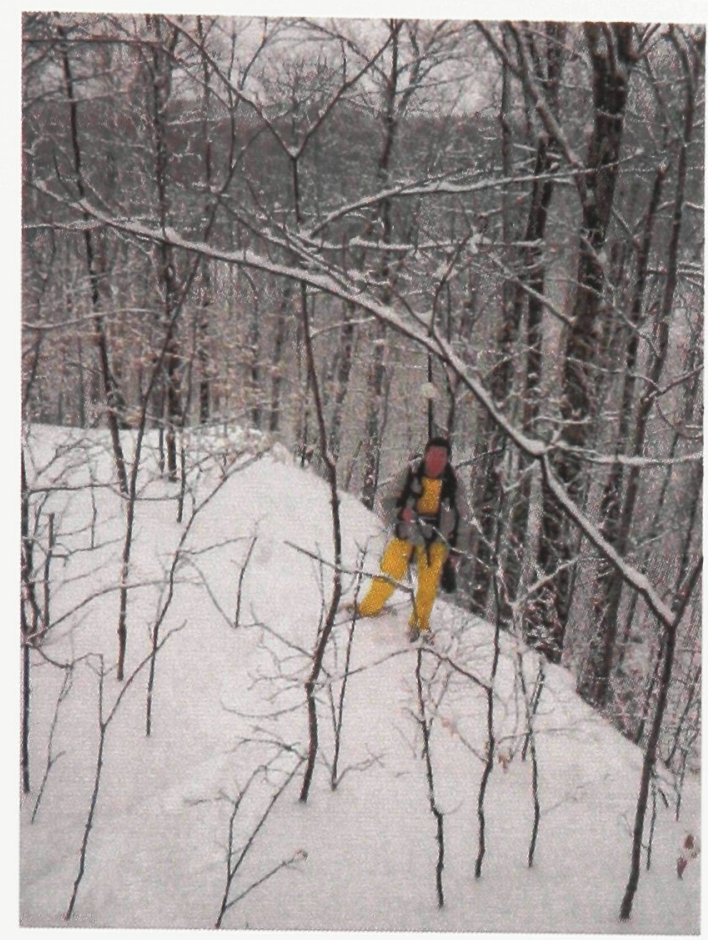

\section{Picture 4.7}

Picture taken during the ground survey conducted during February 2011.

The survey area had a rugged terrain with many steep hills. 


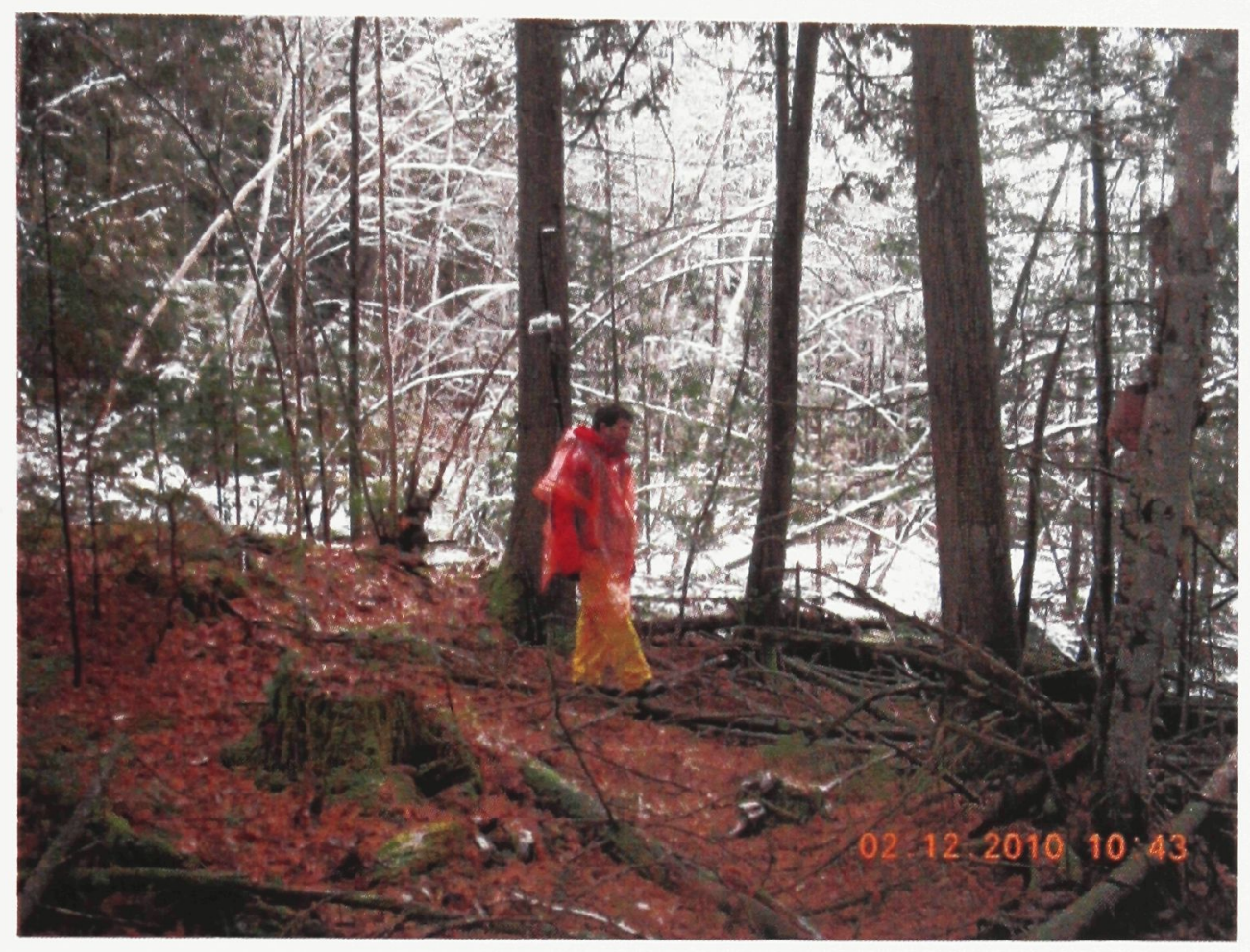

\section{Picture 4.8}

Picture taken during the ground survey conducted during November 2011. The Overhauser GSM19 magnetometer can be seen on the black alumnium pole behind the user. The white GPS antenna can also be seen on top of the pole. 
reception interruptions of the GPS signal. The extent of coverage of both ground surveys can be seen in Figure 4.1.

As both the February and November 2010 surveys were conducted during storms, the GSM-19 Walking and Base units where each respectively shielded from the elements using a plastic poncho, and a Tupperware bin. Both surveys were conducted with two people, with safety equipment that included a bear whistle, a short-wave radio, and a satellite phone. Each person had a specific task during the survey. One was to carry the survey equipment and follow the other person that would use a handheld GPS and a compass to follow the traverse line while negotiating a safe path through the terrain. Care was taken to avoid areas of extreme terrain hazards such as cliffs, and dense bogs.

The ground station was a GSM-19 Base unit and set-up within the survey site. It was installed about $200 \mathrm{~m}$ from the access road, in the woods, and was set-up with a car battery that was certain to provide enough power to last the day. The ground station was situated near traverse line 13, in the center of the survey site. The GSM-19 Base unit did not have GPS capability, it was instead linked each morning with the GSM-19 Walking unit in order to synchronize with GPS time and ensure simultaneous measurements of the magnetic field between units.

The GSM-19 Walking unit was carried on the back with a GPS antenna at the top of a pole. Care was taken to ensure that the measuring system and the person carrying it were free of ferromagnetic materials in order to prevent magnetic interference. 
Measurements were taken at 1 second increments while walking. Episodes of slipping, falling, and stumbling commonly occurred throughout the survey site while surveying. When the falling events were deemed to be excessive, the time and location the fall was recorded so that the corresponding data could be removed during data processing. At the end of the day, the survey gear was packed up and stored. The survey team returned to a motel, and the day's data was downloaded from the GSM-19 Walking and Base acquisition units, and verified for data integrity and completion. Each unit was then charged for use for the following day.

\subsubsection{Data Processing}

A GEM Systems GSM-19 Data Acquisition system was used to record the data. The data acquired includes the data sets listed in Table 4.2. As with the sUAS survey, the $\mathrm{X}, \mathrm{Y}$, and Z co-ordinates were recorded using the World Geodetic System 1984 revision (WGS-84).

A processing flowchart for the ground data is shown in Figure 4.9. The most important steps involve quality control, and IGRF and diurnal corrections. Levelling of the magnetic data set was not required as it was conducted along the ground and so the magnetic field was always recorded at the same height above ground. It was not possible to apply dGPS correction to the GPS data gathered by the GSM-19 Walking unit as the unit is not capable of recording ephemeris data. Applying a lag correction to the data was also not required as the GPS measurements were taken at the same location in space and 
time as the magnetic measurements. The procedures used to verify data quality and to apply IGRF and diurnal corrections to the data, are the same procedures used for the sUAS data and are described in detailed in section 4.1.2. The ground survey was upward continued by $10 \mathrm{~m}$ increments to a height of $50 \mathrm{~m}$ above ground. 
Table 4.2: Table of Data Acquired During the Ground Survey

\begin{tabular}{|l|l|}
\hline \multicolumn{2}{|c|}{ Ground Survey } \\
\hline \multicolumn{2}{|c|}{$\begin{array}{c}\text { GEM-19 Console Data Acquisition System } \\
\text { Records data at 1 Hz }\end{array}$} \\
\hline GSM-19 Overhauser Mag & $0.001 \mathrm{nT}$ \\
\hline GPS Time & $0.01 \mathrm{~s}$ \\
\hline GPS X & WGS-84 at 0.01 m \\
\hline GPS Y & WGS-84 at 0.01 m \\
\hline GPS Z & WGS-84 at 0.01 m \\
\hline \multicolumn{2}{|c|}{ GEM-19 Ground Station } \\
\hline \multicolumn{2}{|c|}{ Records data at 1 Hz } \\
\hline GSM-19 GND Overhauser Mag & $0.001 \mathrm{nT}$ \\
\hline $\begin{array}{l}\text { GPS Time } \\
\text { (synched with walking unit) }\end{array}$ & $0.01 \mathrm{~s}$ \\
\hline
\end{tabular}




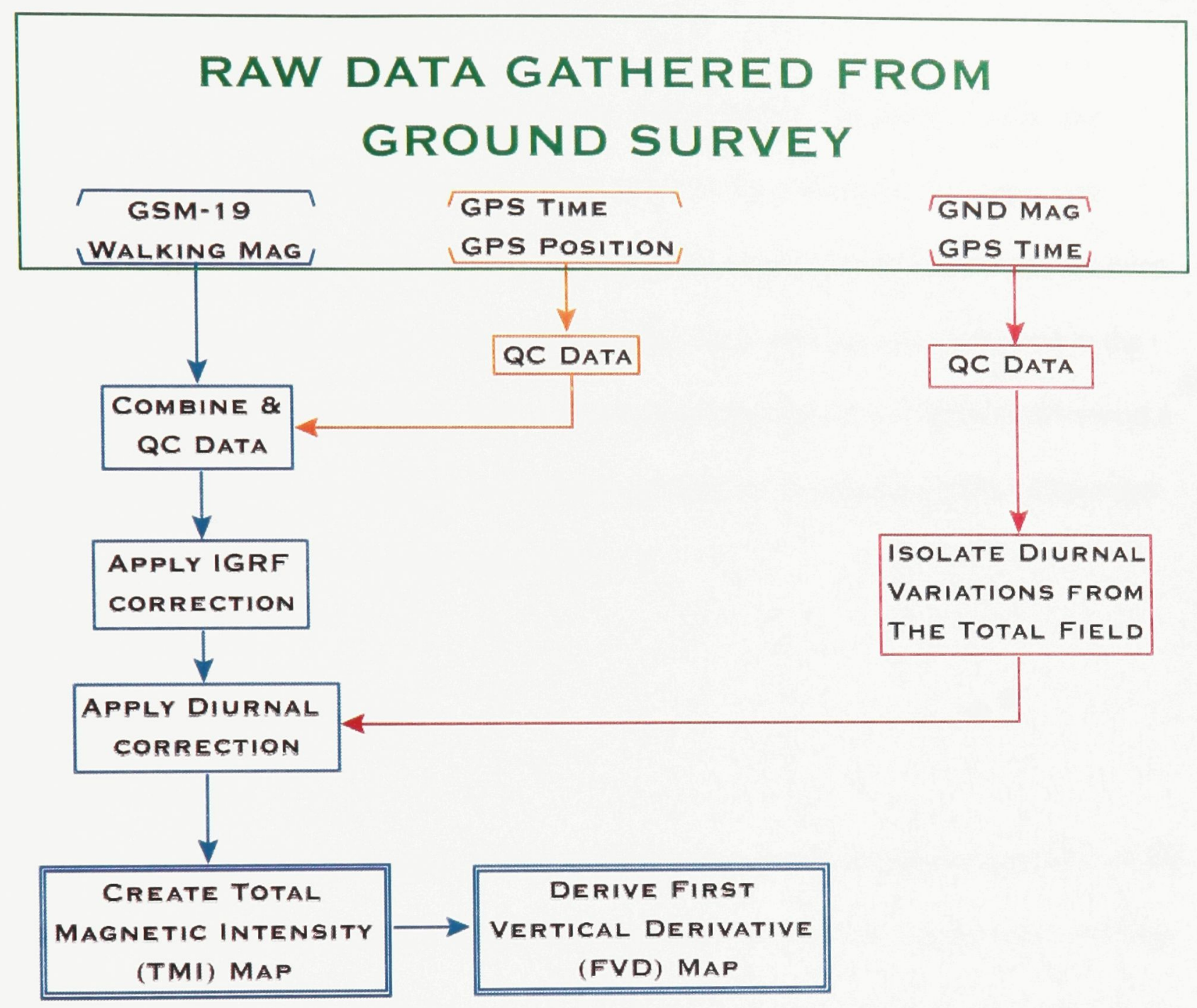

\section{Figure 4.9}

Flowchart showing the various steps performed in order to process the data that were gathered during the ground survey conducted in February and November 2010. The processing resulted in the creation of 2 maps: a TMI map and a FVD map. The GND Mag represents data gathered at a ground station near the survey site. 


\section{Results}

This section presents and discusses the data from the fixed-wing survey, the ground survey, and the sUAS survey, presented in the form of maps. All maps were gridded using the minimum curvature interpolation method. The data presented has been observed or calculated. Observed data corresponds to all data that was measured in the field and subsequently processed. Calculated data corresponds to a map that underwent a grid operation. Grid operations are covered in section 2.4. A summary table of the maps presented in chapter 5 can be found in Table 5.1.

\subsection{Digital Terrain Model}

The digital terrain model (DTM) of the survey site aids in the interpretation of the results of the TMI and gradient maps. The DTM was assembled using the radar and laser altimeter measurements taken from the helicopter. These measurements were subtracted from the mean-sea level GPS altitude data. The result of the subtraction provides the relief of the terrain with respect to mean sea level. As the altimeter measurements were taken during the winter, the altimeters did not detect the leafless deciduous trees, but did measure coniferous trees. The presence of each coniferous tree was removed using a band-pass filter. The filter identified the individual trees by their average size. In the survey area, the average coniferous tree was up to $20 \mathrm{~m}$ tall and up to 5 meters wide. 
Table 5.1: Summary Table of Maps and Data Sets

\begin{tabular}{|l|l|l|l|}
\hline Map Type & Survey & Type & Nominal Altitude \\
\hline TMI & Fixed-Wing & Observed & $150 \mathrm{~m}$ \\
\hline TMI & Ground & Observed & $0 \mathrm{~m}$ \\
\hline TMI & Ground & Calculated & $50 \mathrm{~m}$ \\
\hline TMI & sUAS & Observed & $50 \mathrm{~m}$ \\
\hline FVD & Fixed-wing & Observed & $150 \mathrm{~m}$ \\
\hline FVD & Ground & Calculated & $0 \mathrm{~m}$ \\
\hline FVD & sUAS & Calculated & $50 \mathrm{~m}$ \\
\hline Longitudinal & sUAS & Observed & $50 \mathrm{~m}$ \\
\hline Longitudinal & sUAS & Calculated & $50 \mathrm{~m}$ \\
\hline Transverse & sUAS & Observed & $50 \mathrm{~m}$ \\
\hline Transverse & sUAS & Calculated & $50 \mathrm{~m}$ \\
\hline Data Set & Survey & Type & Nominal Altitude \\
\hline Transverse & sUAS, line 1010 & Observed & $100 \mathrm{~m}$ \\
\hline Transverse & sUAS, line 1010 & Observed & $150 \mathrm{~m}$ \\
\hline Transverse & sUAS, line 1010 & Observed & $200 \mathrm{~m}$ \\
\hline & & &
\end{tabular}


The DTM shown in Figure 5.1 incorporates a basemap of the survey area which identifies lakes and roads. The DTM and the SRTM map, shown in Figure 4.1, are similar as both depict topographical variations throughout the survey site. The primary difference between the two is the resolution at which the data was obtained. Where the SRTM data was gathered from space, the DTM data was gathered within $100 \mathrm{~m}$ of the ground. The cell size of the DTM is $10 \mathrm{~m}$, while the cell size of the SRTM map is $90 \mathrm{~m}$. On the DTM shown in Figure 5.1, the features marked by the numbers 1 to 5 will be referred to throughout the results section. Feature 1 indicates the location of a linear topographical ridge that is striking to the north-east. Feature 3 indicates the location of a variable terrain that has a large number of hills, and feature 4 indicates the location of a valley that cuts between two linear ridges that share a north-eastern strike. Feature 4 is actually the topographic expression of the Plevna Fault as it passes through the survey site. It can be correlated to the inferred location of the fault in Figure 3.2. Features 5 and 2 create a valley that runs between the feature 1 ridge and another ridge in the northernmost section of the map. This valley is predominantly covered by a bog.

\subsection{Total Magnetic Intensity Maps}

TMI maps of the survey area were created from the fixed-wing, sUAS, and ground survey data sets. The fixed-wing data set was gridded with a $40 \mathrm{~m}$ cell size as the traverse line spacing was $200 \mathrm{~m}$. The TMI map of the fixed-wing survey shown in Figure 5.2 shows magnetic highs, indicated by red colours, in the northern and southern portion of the map. Figure 5.3 shows that these highs correspond to the location of the 


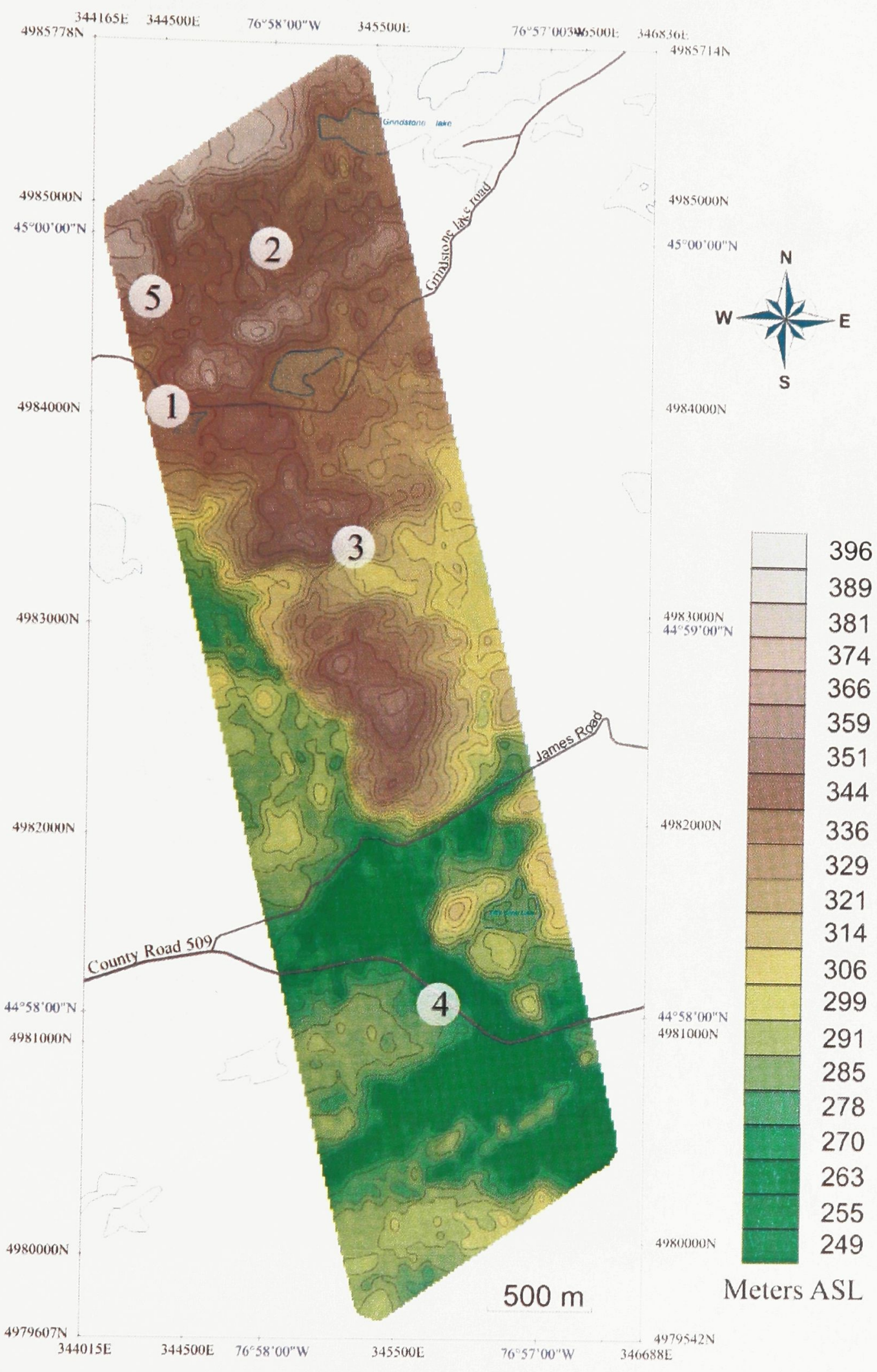

Figure 5.1

Digital terrain model (DTM) of the survey site. Colours scale shows terrain relief above mean sea level. 


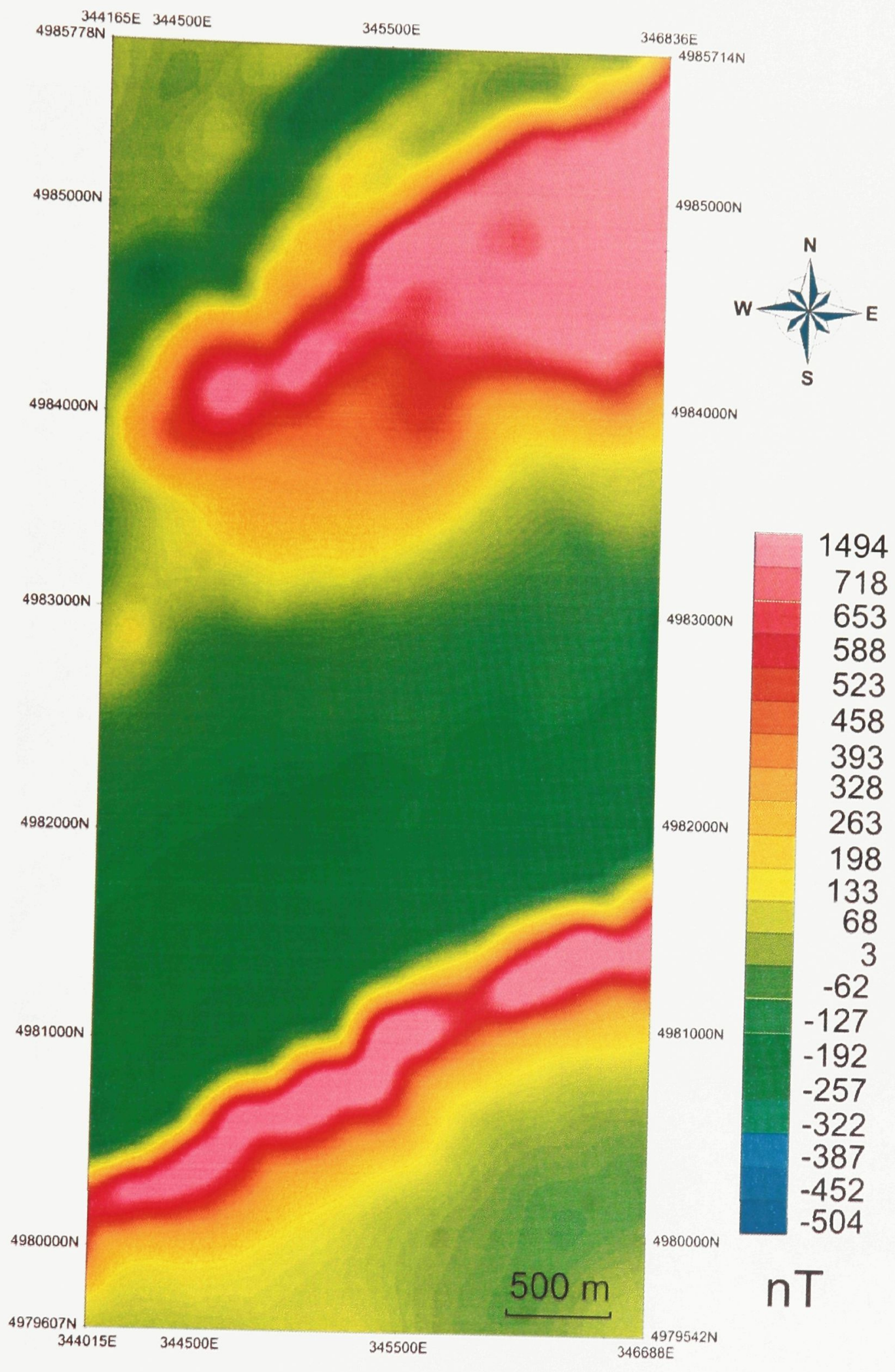

\section{Figure 5.2}

Map of total magnetic intensity gathered by a conventional fixed-wing aircraft. The map is based on data gathered at an altitude of $150 \mathrm{~m}$ above ground. Map cell size is $40 \mathrm{~m}$. 


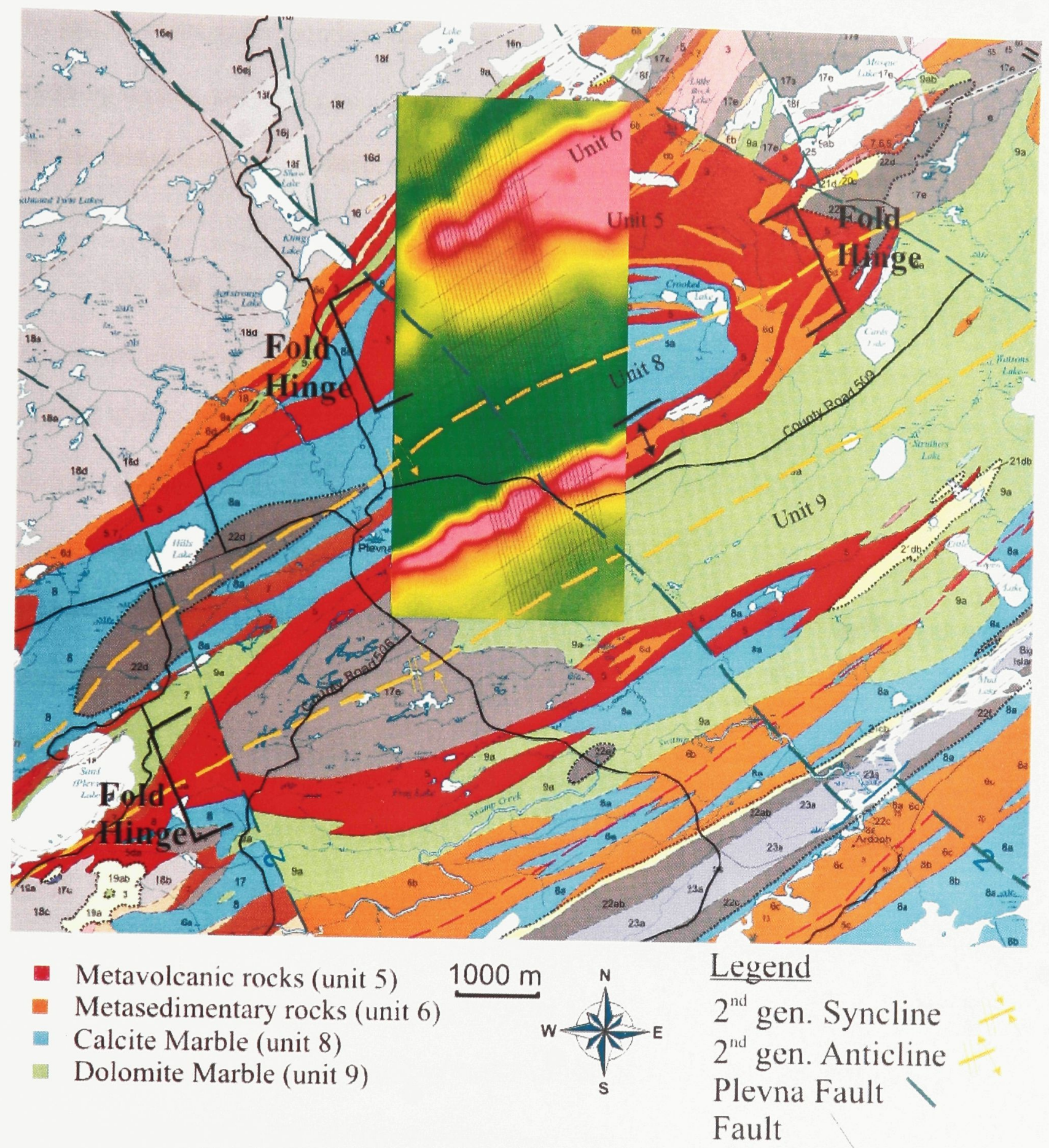

\section{Figure 5.3}

Fixed-wing TMI map superimposed on the modified structural geology map. 
metavolcanic rocks of unit 6 . The figure also shows that the magnetic lows, indicated by the green colour, corresponds to the location of the calcitic and dolomitic marbles that make up units 8 and 9. The contrasting colours of the TMI map indicate the location of the fold hinge and the fold limb, which is in agreement with the structural geology map.

The TMI map created from the $50 \mathrm{~m}$ altitude sUAS survey data, shown in Figure 5.4, covers a smaller portion of the TMI map of Figures 5.2 and 5.3. This map was gridded with a cell size of $10 \mathrm{~m}$. As with the fixed-wing TMI map of Figure 5.2, the prominent magnetic highs and lows in this map correspond to the location of the metavolcanic rocks of unit 6 , and the marble rocks of units 8 and 9 . Immediately noticeable between Figures 5.2 and 5.4 is that they look similar. They show many of the same features at a similar magnetic intensity. Also apparent is the improved level of detail of Figure 5.4 over Figure 5.2, due to the lower altitude at which the magnetic data was acquired.

The TMI map created as a result of the ground surveys conducted in February and November 2010 can be seen in Figure 5.5. As mentioned in section 4.2.1, the coverage of the ground survey was not as extensive as that of the sUAS survey. The difference in the appearance of the TMI ground survey from that of the sUAS and the fixed-wing survey is due to the high frequency content of the magnetic intensity data that was recorded along the ground. The finer detail in the ground survey map has the greatest potential to delineate the smallest magnetic trends. It is this map that will be used as a reference to detect magnetic trends that may not be visible in other maps. 


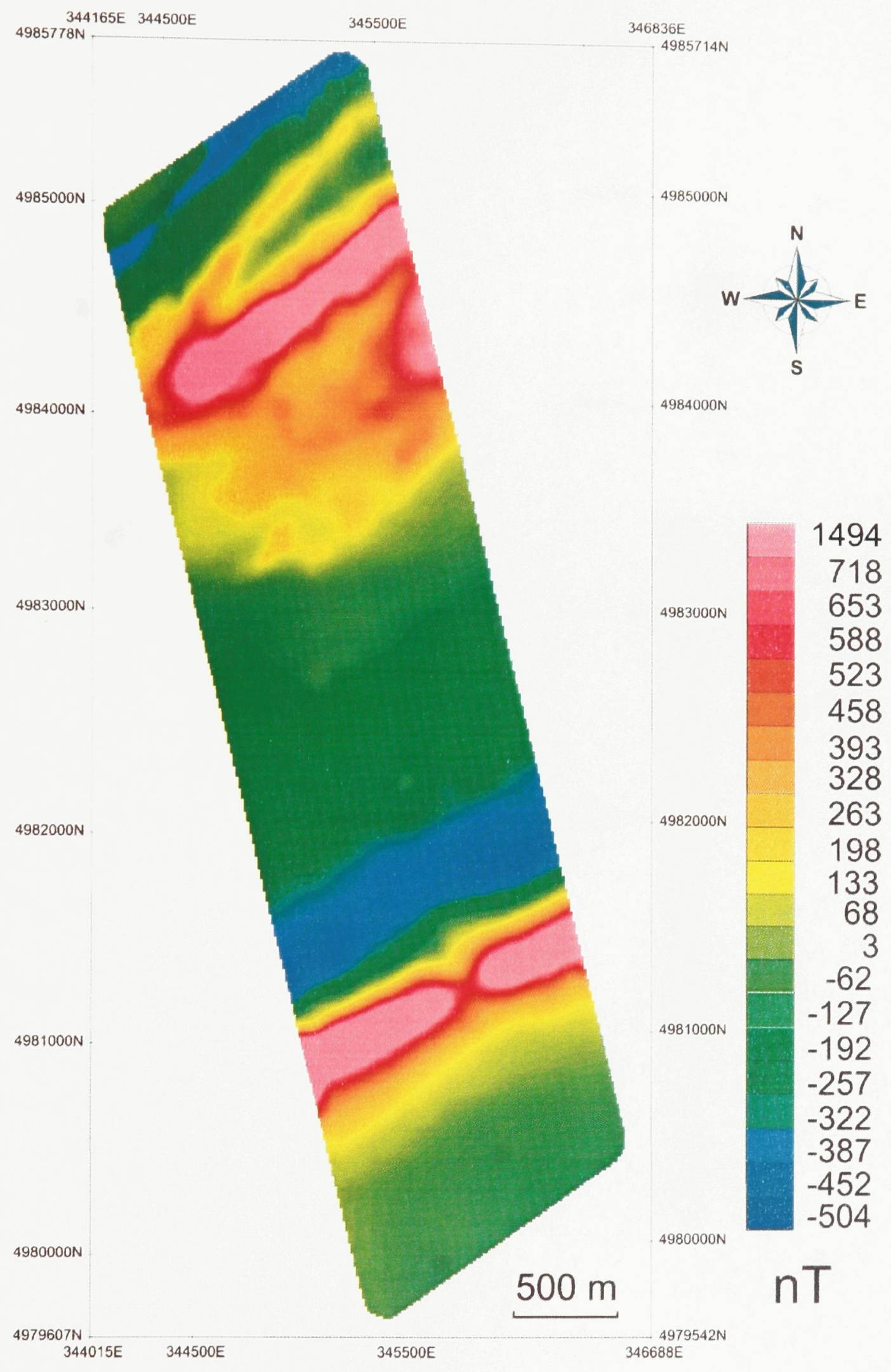

\section{Figure 5.4}

Map of total magnetic intensity gathered by the simulated UAS. The colour scale is identical to that used in Figure 5.2 to provide comparison. Map cell size is $10 \mathrm{~m}$. 


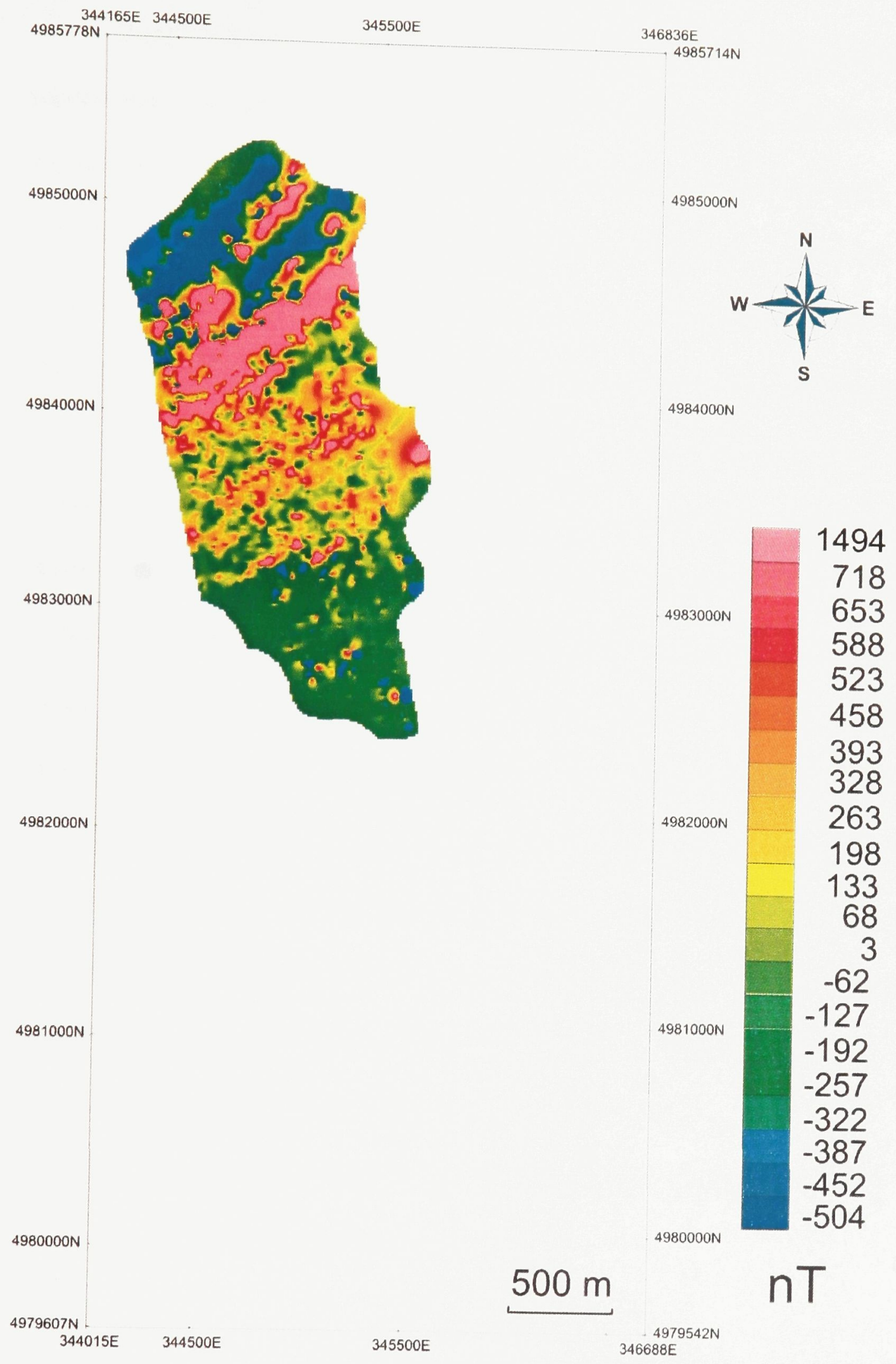

\section{Figure 5.5}

Map of total magnetic intensity gathered by the ground survey. The colour scale is identical to that used in Figures 5.2 and 5.4 to provide comparison. Map cell size is $10 \mathrm{~m}$. 
Figure 5.6, shows a sequence of upward continuations applied to the TMI ground survey data from ground level to an altitude of $50 \mathrm{~m}$. Figures 5.5 and 5.6 were created with a cell size of $10 \mathrm{~m}$ for direct comparison against the sUAS TMI map. The first map, with measurements at ground level is the same map depicted in Figure 5.5. The sequence of upward continuations illustrates the change in frequency content in the ground magnetic data with increasing altitude. Each map delineates the fold hinge, which is shown by the concentration of magnetic highs. At ground level, however, the data also exhibits many local bulls-eye anomalies that can be attributed to both signal and noise. Many of these bulls-eye anomalies are created from variations in measurements that are a by-product of the walking acquisition method. They were exacerbated due to the rugged nature of the terrain, the shallow depth to bedrock, and the high intensity and rapid variations of the magnetic field. Movements of the magnetometer, introduced despite best efforts, generated high frequency high amplitude variations, on the order of 20-30 nT, within the data. These variations were indistinguishable from incidents where the carrier tumbled or slipped, and so this source of error could not be corrected despite having the time of each occurrence recorded. The widespread occurrence of these features and the ambiguity of their origin complicate interpretation by cluttering the map. As altitude increases, the very high frequencies are attenuated to reveal more meaningful magnetic trends that originate from deeper sources.

A comparison of the magnetic intensity recorded by the ground and the sUAS survey along traverse line 1010 in Figure 5.7 reveals the large amount of high frequencies measured by the ground survey (see Figure 5.6 for the location of line 1010). The high 


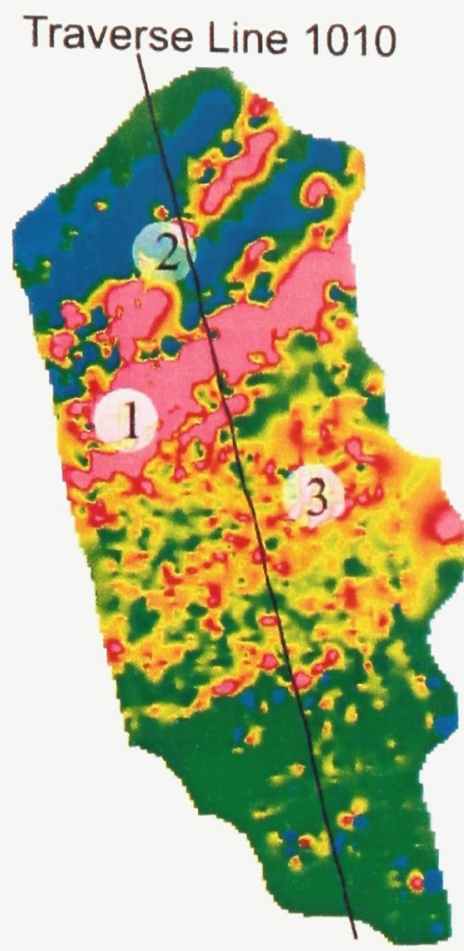

Ground Level

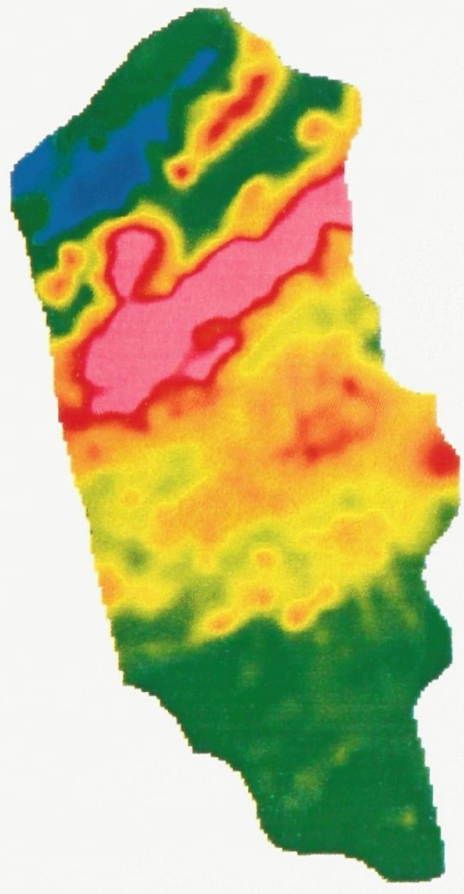

$30 \mathrm{~m}$

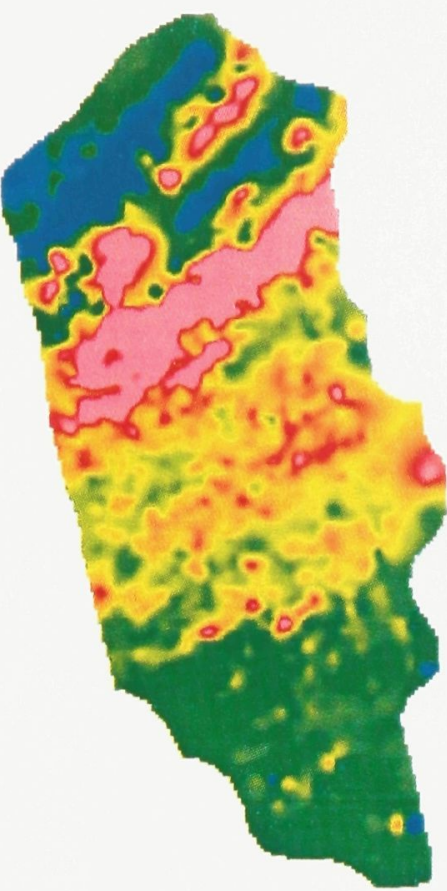

$10 \mathrm{~m}$

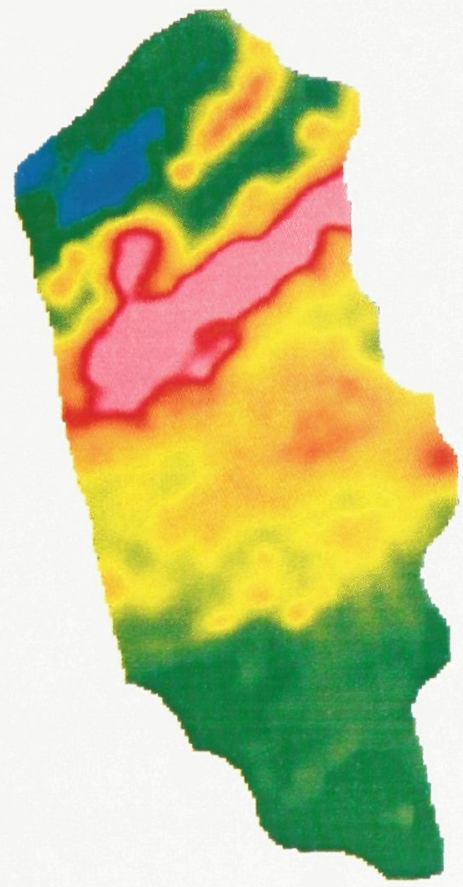

$40 \mathrm{~m}$

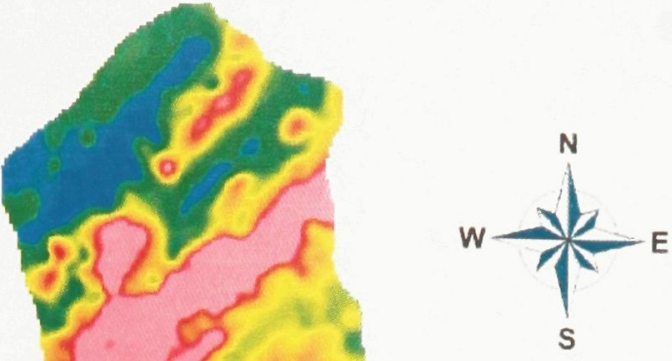

$500 \mathrm{~m}$

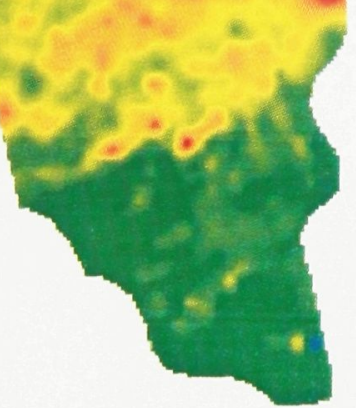

$20 \mathrm{~m}$

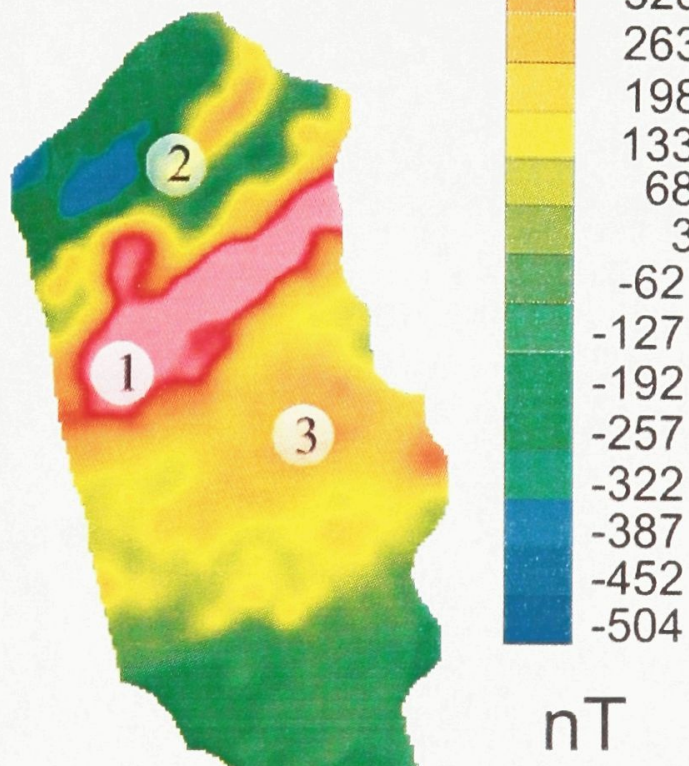

133

68

3

$-62$

$-127$

$-192$

$-257$

$-322$

$-387$

$-452$

$-504$

$\mathrm{nT}$

Figure 5.6

TMI ground survey maps that have been progressively upward continued from ground level to an altitude of $50 \mathrm{~m}$ in increments of $10 \mathrm{~m}$. These maps use the same colour scale and are to be compared with the fixed-wing and sUAS TMI maps shown in Figures 5.2 and 5.4. 


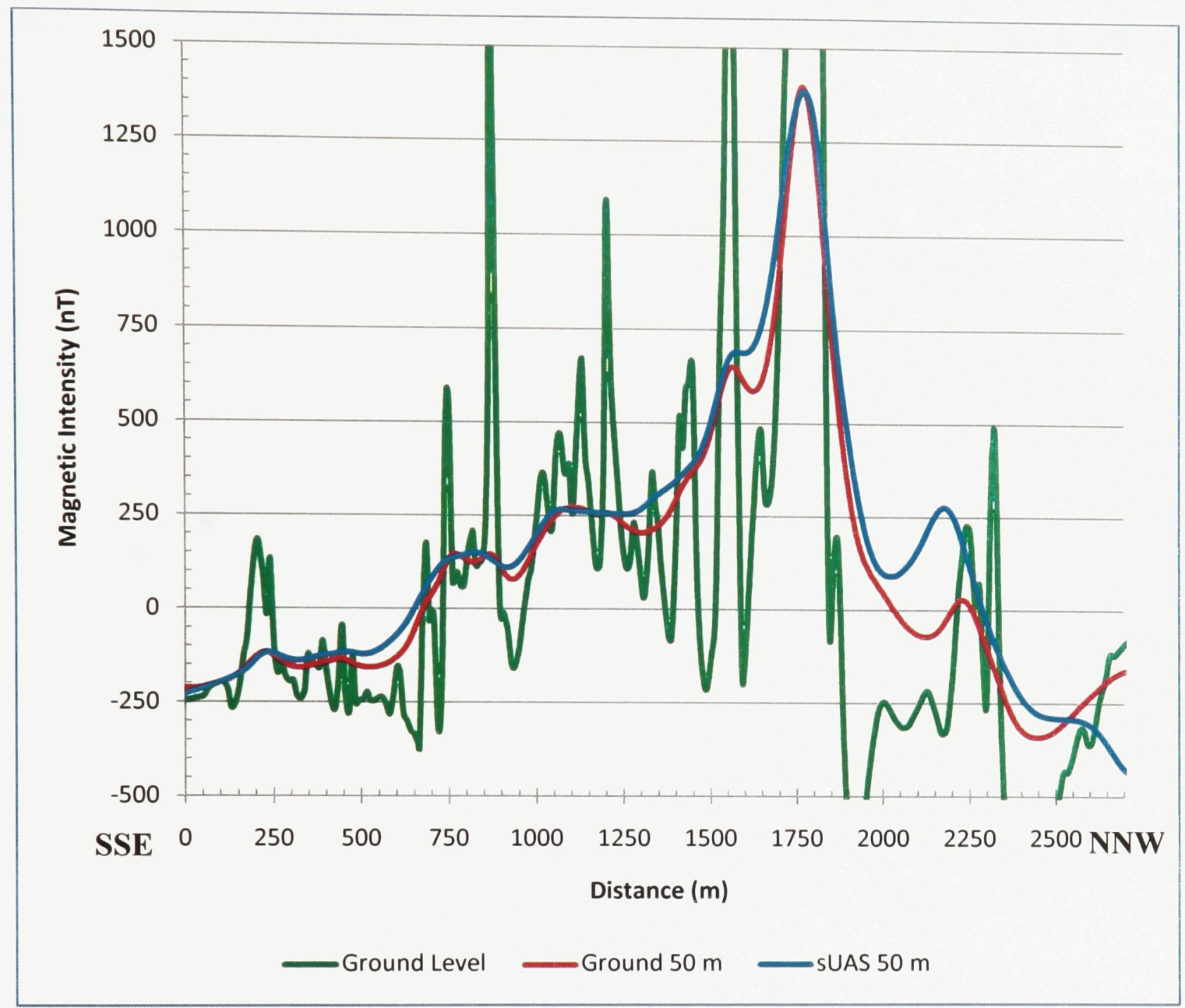

\section{Figure 5.7}

Comparison of magnetic intensity between the ground survey (green line) and sUAS survey (blue line) along traverse line 1010 (see location in Figure 5.6). Plot reveals the high frequency content of the data recorded during the ground survey. It also shows the high-cut filtering effect caused by upward continuation to an altitude of $50 \mathrm{~m}$ (red line). 
amplitude and high frequency of the peaks measured by the ground survey are such that they obscure the magnetic trend created by the geological units present in the vicinity of the fold hinge. The peak with the highest amplitude, which starts at $1750 \mathrm{~m}$, exceeds $1500 \mathrm{nT}$, and has been truncated in Figure 5.7, corresponds to the linear magnetic high of feature 1 in Figure 5.5. Feature 1 can be identified from the DTM in Figure 5.1 and is a topographic anomaly. A topographic anomaly is a magnetic feature created by the hills and valleys within a terrain. If the effect of the feature 1 is to be ignored, then Figure 5.7 shows another magnetic high with a long wavelength. This high starts at about $600 \mathrm{~m}$ distance and ends at about $1900 \mathrm{~m}$, where the magnetic intensity in the graph rises above $0 \mathrm{nT}$. Although it can be identified in all three profiles, it is delineated more clearly on the upward continued ground data, shown in red, or the sUAS data shown in blue. This magnetic high corresponds to the fold hinge shown in Figure 3.2 and 5.3, which is the location where the high susceptibility rocks of unit 6 are located.

A comparison of the fixed-wing, sUAS TMI, and upward continued to $50 \mathrm{~m}$ altitude ground survey (UP50m), maps in Figure 5.8 show the similarities between maps. The magnetic high of the fold hinge can be found easily within each map, situated between the magnetic lows that correspond to marble units 8 and 9. Features 1 and 3 can also be visually correlated between maps. Also obvious is the expected increase in resolution at lower altitude. The sUAS TMI map and the UP50m map are each showing a higher level of detail compared to the fixed-wing map. The most prominent example of the increase in resolution can be seen by comparing feature 1. In the fixed-wing map this feature can be identified to be linear in nature, but lacks the higher detail found in the 


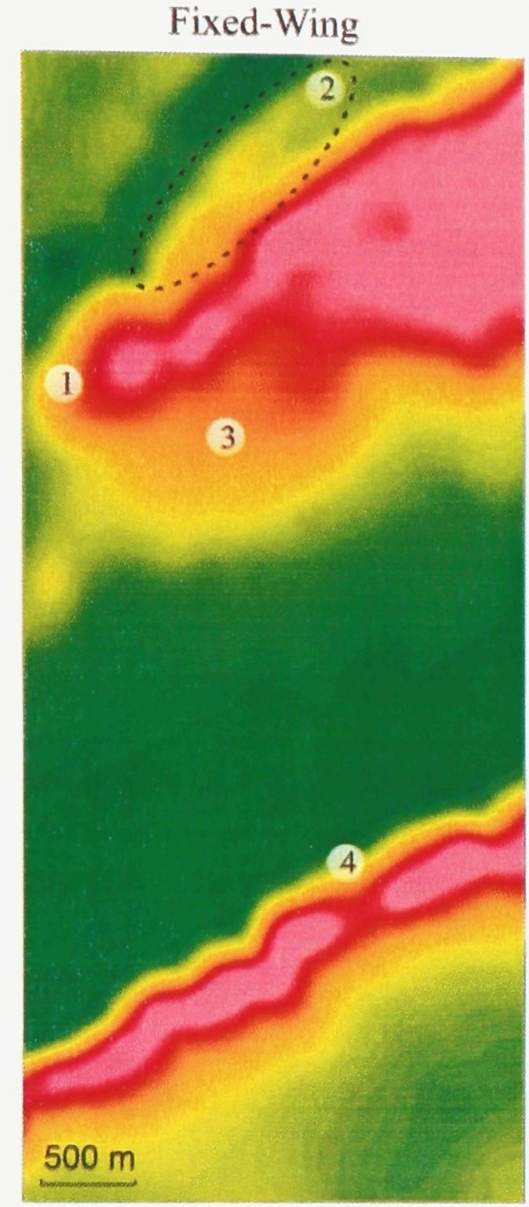

$150 \mathrm{~m}$ altitude

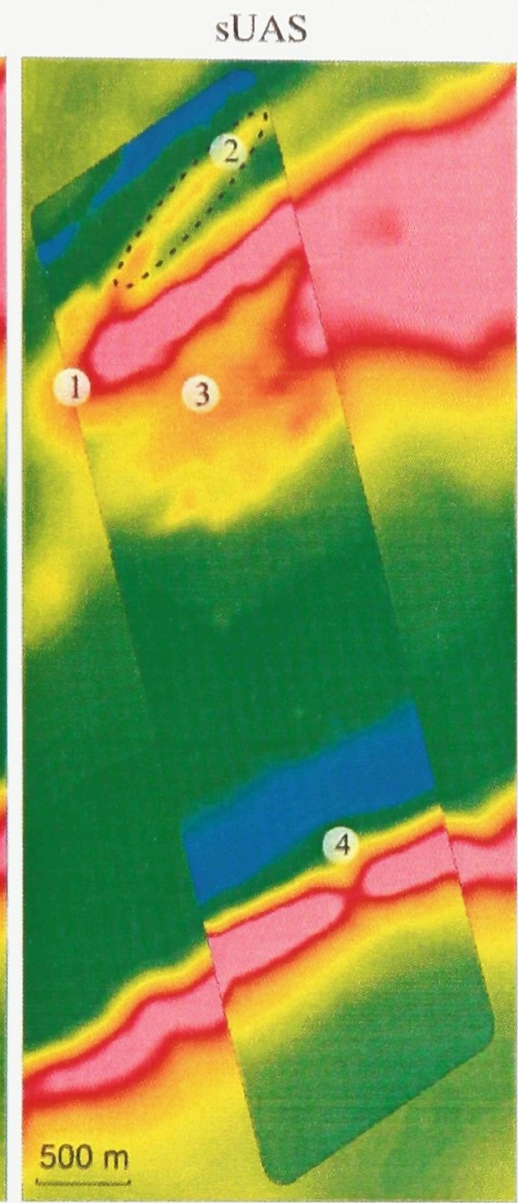

$50 \mathrm{~m}$ altitude

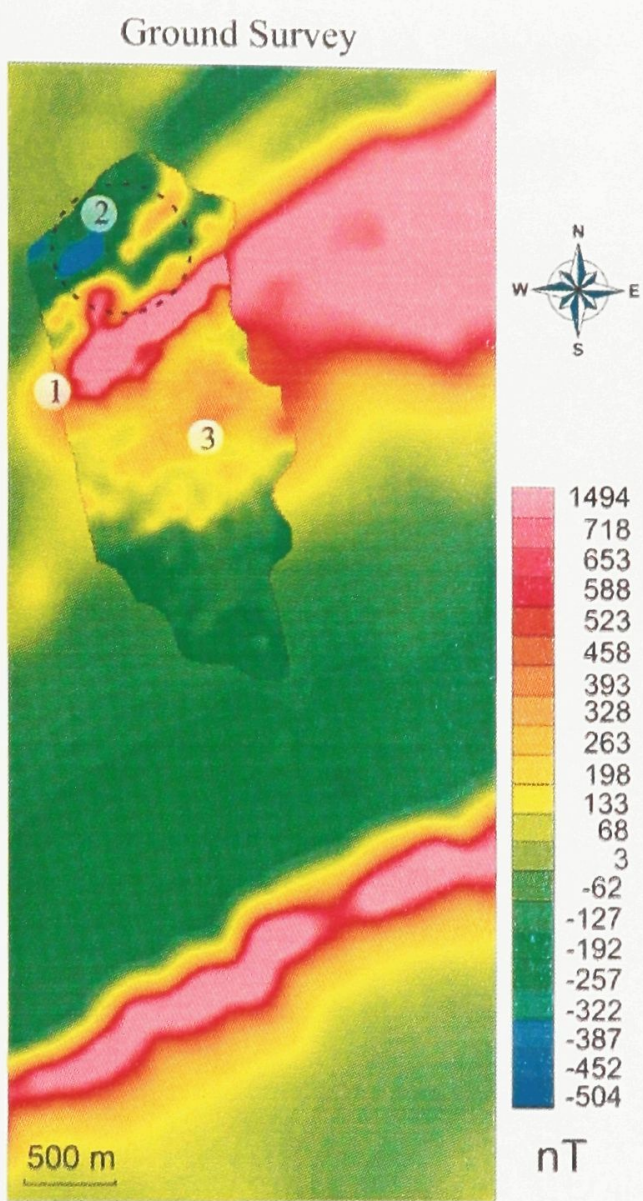

upward continued $50 \mathrm{~m}$

\section{Figure 5.8}

Comparison of TMI maps from the fixed-wing, the sUAS survey, and the upward continued to 50

$\mathrm{m}$ (UP50m) altitude ground survey. Both the sUAS and the UP50m maps are superimposed on the fixed-wing map. 
sUAS and the UP50m maps. Another example of the increase in detail of the sUAS and the UP50m over the fixed-wing survey can be seen by comparing the topographic anomaly of feature 3 . There is more detail in the location of the various hills, which can be identified as hills using the DTM in Figure 5.1. For feature 4, both the fixed-wing and the sUAS maps delineate the fold hinge to almost the same degree. The dip in magnetic intensity near the marker for feature 4 is an indication of another topographic anomaly, this time created by the presence of a valley, which is identified in the DTM of Figure 5.1 .

It is important to note that the differences in continuity between the fixed-wing map, the sUAS map, and the UP50m map, which can be seen by comparing the linear trends of features 1 and 4, is due to the use of GPS referenced measurements which was not a technology available during the fixed-wing survey. The absence of this technology contributes to an additional lag effect that cannot be corrected as the survey acquisition system is unable to record the geo-referenced location of the fixed-wing aircraft. This information is required to determine the geo-referenced location of the magnetometer in time and space.

It is expected that the sUAS map and the UP50m map of Figure 5.8, both shown at a $50 \mathrm{~m}$ altitude, would show the same level of detail. The figure however, shows that the UP50m map has slightly more detail than the sUAS map. This can be seen by comparing features 1 and 3 . This is due to differences in distance to the bedrock from which the magnetometer made the measurements during each survey. For the sUAS 
survey the distances are variable due to the drape that the helicopter was flying. This concept is explained in detail in section 4.1.1.

Also illustrated in Figure 5.8 is one of the benefits of aeromagnetic surveying over ground surveying. There is a linear magnetic high identified by feature 2 , and outlined by a dotted line, in the fixed-wing and sUAS maps. In the UP50m map, this feature appears boudinaged as opposed to linear due to the presence of a $75 \mathrm{~m}^{2}$ gap in the survey data. This gap corresponds to the location of a bog that was not traversed for safety reasons. During the creation of the map, the missing data was interpolated and the feature was not well represented. This could lead to an inaccurate interpretation of the magnetic trend and the geology in that area.

A comparison of Figure 5.8 with Figure 5.6 reveals that the sUAS map contains all of the magnetic trends visible in not only the UP50m map, but all of the upwardcontinued ground survey maps in Figure 5.6. In the sUAS map, features 1 and 2 are resolved to a similar extent, and feature 3 has increased clarity. Since there are no magnetic trends missing between the ground survey and sUAS maps, no new trends will be detected should the flight altitude of the sUAS be lowered below $50 \mathrm{~m}$. In the absence of the forest, if the sUAS survey had been conducted at a lower altitude, using the same traverse line spacing, then the higher resolution data obtained would not actually reveal previously unknown trends and would have not contributed additional value to a geological interpretation. 


\subsection{Vertical Gradient Maps}

A comparison using the vertical gradient of the intensity of the magnetic field between the fixed-wing, sUAS, and ground survey is shown in Figure 5.9. The fixedwing map shows the observed vertical gradient, and is compared with the SUAS FVD, and the $50 \mathrm{~m}$ ground survey FVD. Since the data sets were all gathered at different nominal altitudes, the corresponding intensity of the magnetic gradient will be different, as explained in section 2.2. For this reason, the maps could not be plotted using the same colour scale. The colours scales used to create each map are based on equal area, and so the change in a colour corresponds to a change in vertical gradient. This allows a qualitative visual comparison. In all cases, the FVD was calculated using the Fourier method.

At first glance there are many similarities between the maps. The fixed-wing, sUAS, and ground survey maps all reproduce features 1,2, and 3. As with the TMI maps, the sUAS and the ground survey FVD maps are able to show these features at a higher level of detail due to the lower altitude at which the surveys were conducted or upward continued to. There are no magnetic trends occurring in the ground survey map that cannot be identified in the sUAS map.

Differences between the fixed-wing and the sUAS map will be discussed first by comparing feature 1 . The fixed-wing map delineates this strong linear trend but is unable to resolve features 1 and 2 when they are in close proximity to each other. The result is a 


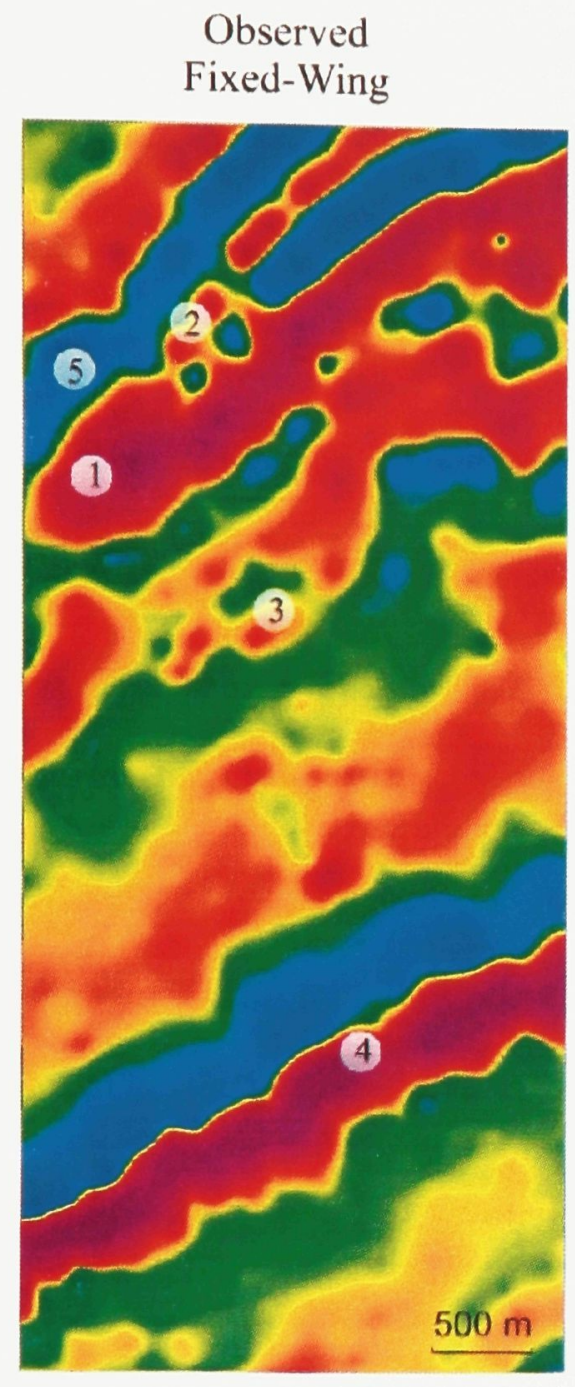

$150 \mathrm{~m}$ altitude
Calculated

sUAS

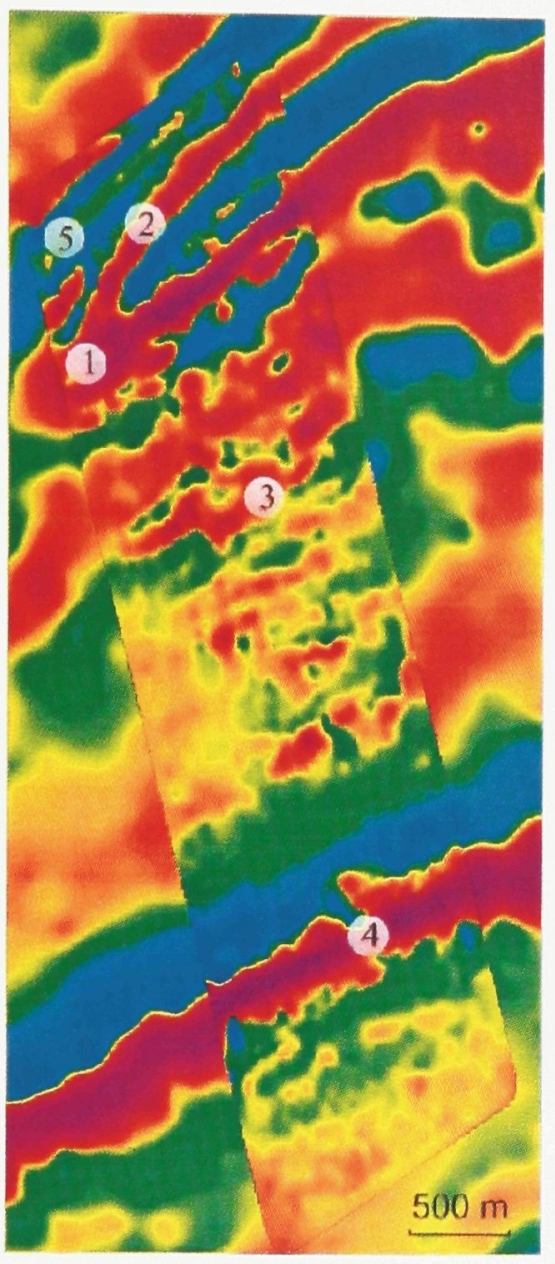

$50 \mathrm{~m}$ altitude
Calculated

Ground Survey

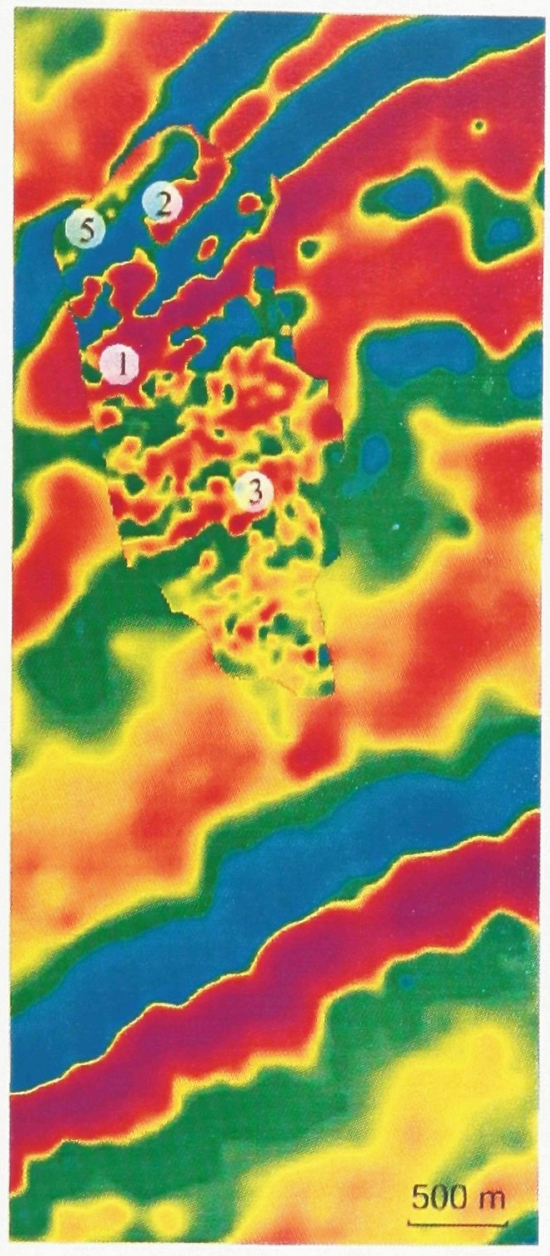

upward continued $50 \mathrm{~m}$

\section{Figure 5.9}

Comparison of the observed vertical gradient from the fixed-wing survey, the FVD from the sUAS survey, and the UP50m altitude FVD. The color scale is not the same for the three maps. Colors have been chosen to allow for a qualitative comparison between the maps. Both the sUAS FVD and the UP50m FVD maps are superimposed on the fixed-wing FVD map. 
fusion of the magnetic signature of each feature. Feature 2 also appears boudinaged as opposed to the continuous linear feature that is illustrated in the sUAS map. Feature 3 can also be seen to be more variable in the sUAS map as opposed to the fixed-wing map. Both maps reveal feature 4 to be a linear magnetic high striking to the north-east. There is however a prominent difference in shape of this feature between the two maps. The sUAS map shows a discontinuity to the magnetic gradient at the exact location that corresponds to the valley depicted in the DTM in Figure 5.1. In a valley, a portion of the magnetic field being measured comes from the ridges on either side, and so the vertical gradient will be variable in extent and intensity, and sensitive to the change in the topography. Another feature absent in the fixed-wing map is feature 5. Feature 5 is a narrow linear magnetic high with a similar strike as feature 2. It occurs within a valley, illustrated in the DTM in Figure 5.1, that both features 5 and 2 share. Since the DTM is showing no topographical variations that match either of these features, they can be identified as magnetic anomalies and are most likely created from a geological structure in the subsurface. Examining the ground survey map in Figure 5.9 does not reveal this feature in the same level of detail as the sUAS map. This is most likely due to the extent of the survey coverage as the anomaly appears as a series of magnetic high bulls-eyes on the northern edge of the ground survey map. As with feature 2 , if the coverage of the ground survey had been as extensive as that of the sUAS survey, the magnetic anomaly of feature 5 would most likely show a similar level of detail. The geology in that section of Figure 3.2 is complex with rock units 6 and 9 alternating in occurrence while sharing the same strike to the north-east. There are no other significant differences between the sUAS map and the ground survey map in Figure 5.9. 


\subsection{Transverse and Longitudinal Gradient Maps}

The second objective of this project is to analyze the functionality of the GeoSurv II transverse gradiometer. The sUAS results can be seen in Figure 5.10 (left). The transverse derivative is shown in Figure 5.10 (right) to provide a comparison. The advantage of a transverse gradiometer is to mainly resolve magnetic trends that are parallel to flight direction. Unfortunately, trends in this orientation are not present in either of the transverse maps in Figure 5.10, and so other trends will be used to describe the transverse gradiometric data.

Immediately apparent in Figure 5.10 is the similarity between the observed transverse gradient, and the transverse derivative. Features 1 to 5 are prominent and can be easily correlated between maps. Since the transverse derivative is created by interpolating the change in the intensity of the residual magnetic field from the TMI map, which itself has been interpolated between survey lines, the interpolation is limited by the traverse line spacing of $50 \mathrm{~m}$. The lower resolution of the transverse derivative map is subtle, but appears in the small differences within each feature.

The difference in the shape of feature 1 , which has been interpreted to be a topographic anomaly, illustrates the higher resolution of the observed traverse gradient over the transverse derivative. The DTM figure shows the ridge to have a variable slope as it strikes to the north-east. The change in slope angle and orientation is probably due to differences in erosion rates along various faces of the ridge. The result is a ridge that has 


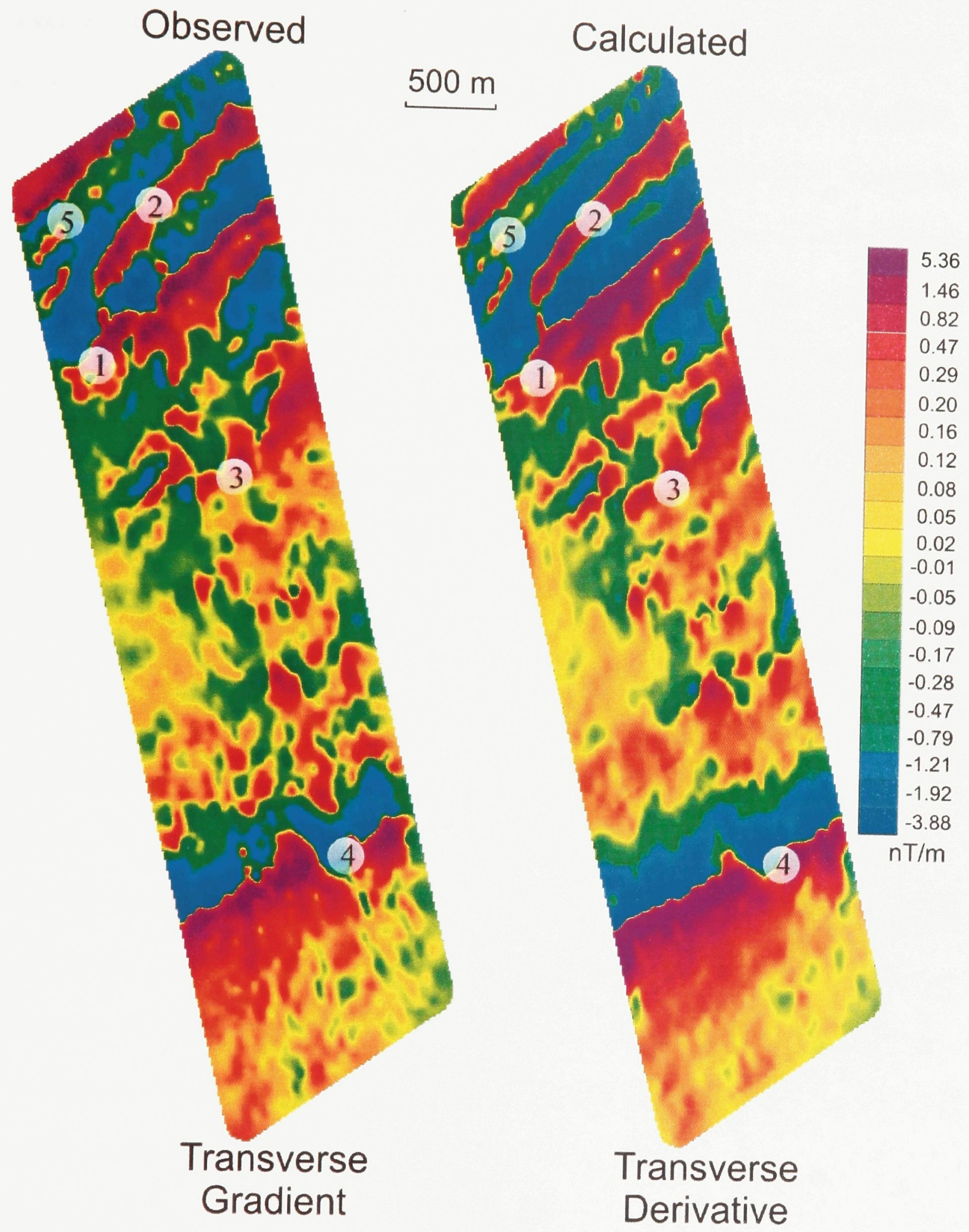

\section{Figure 5.10}

Comparison of the observed transverse gradient and the calculated transverse derivative of the TMI map from the sUAS survey. 
a general strike to the north-east, but a cliff face and slope that may jut out, or become recessed relative to the general ridge. Since the ridge is irregular in shape, it should be expected for the magnetic field generated from that ridge to be non-uniform in shape as well. The observed gradient map in Figure 5.10 (left) shows a non-uniform distribution of the magnetic field that is similar to the variations observed on both sides of the ridge in the DTM. Some of these changes can be attributed directly to variations of the cliff face in the ridge itself. On the other hand, the transverse derivative in Figure 5.10 (right) shows a straight and uniform magnetic field with few deviations, a representation unsupported by the DTM. The transverse derivative did not capture the character of the ridge with the same fidelity as the observed gradient.

The high level of agreement between the two maps in Figure 5.10, in terms of the location and shape of each feature, suggest that the measured transverse gradient is reliable. This point is reinforced when considering Figure 5.11, which compares the observed transverse gradient with the FVD. Features 1,2, and 5 correspond well between maps in terms of shape and location. Feature 3 shows the same type of variability that is to be expected from an area that is covered in hills, as shown in the DTM in Figure 5.1. The location of the fold limb can be correlated between maps, and the area around feature 4 depicts a complicated area in the same location in each map. The changes in the magnetic field in this location is interpreted to reflect the local geology where the valley cuts through the ridge. 

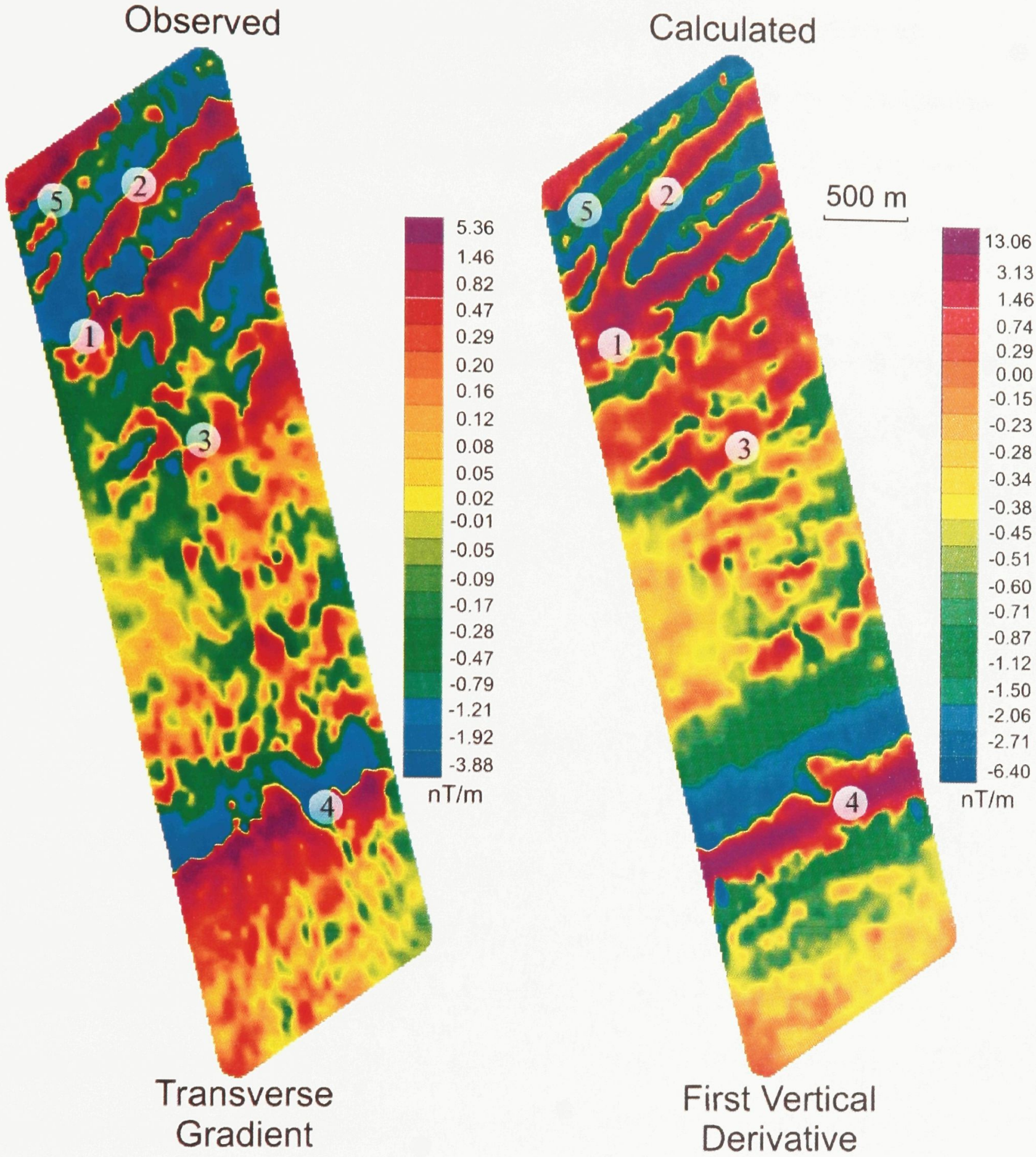

\section{Figure 5.11}

Comparison of the observed transverse gradient and the calculated sUAS FVD gradient. 
In Figure 5.12, the observed transverse gradient can be compared with the observed longitudinal gradient. The method used to determine the observed longitudinal gradient is discussed in section 2.2. The longitudinal gradient resolves magnetic trends that are perpendicular to flight direction, and so any correlation between maps should be focused on oblique NE-SW trends. Features 1,2, and 5 are oblique trends that can be correlate well between maps. The interpretation of feature 3 is inconclusive because the changes in the magnetic field are not only due to changes in rock type, as observed in Figure 5.3, but also to superimposed topographic anomalies which are shown in the DTM in Figure 5.1. Feature 4 is more parallel to the direction of the flight line and so will show little expression on a longitudinal map.

Previous magnetic surveys conducted around the survey site did not detect the Plevna fault (Pauk 1982). The sUAS TMI, FVD, and transverse gradient results support this observation as there is no NW-SE magnetic trend that can be observed on any of the maps. In the vertical gradient maps, in Figure 5.9, and the horizontal gradient maps, in Figures 5.10 to 5.12 , some variations in the magnetic field around feature 4 are most likely attributed to variations in the topography as opposed to the magnetic signature of the fault. Changes in the magnetic field start and end with corresponding changes in topography. As the Plevna fault has been identified to be variable in magnetic intensity, the absence of any magnetic trend that can be attributed to the fault may have more to do with the magnetic signature of the fault within the survey area as opposed to the sensitivity of the gradiometer. 

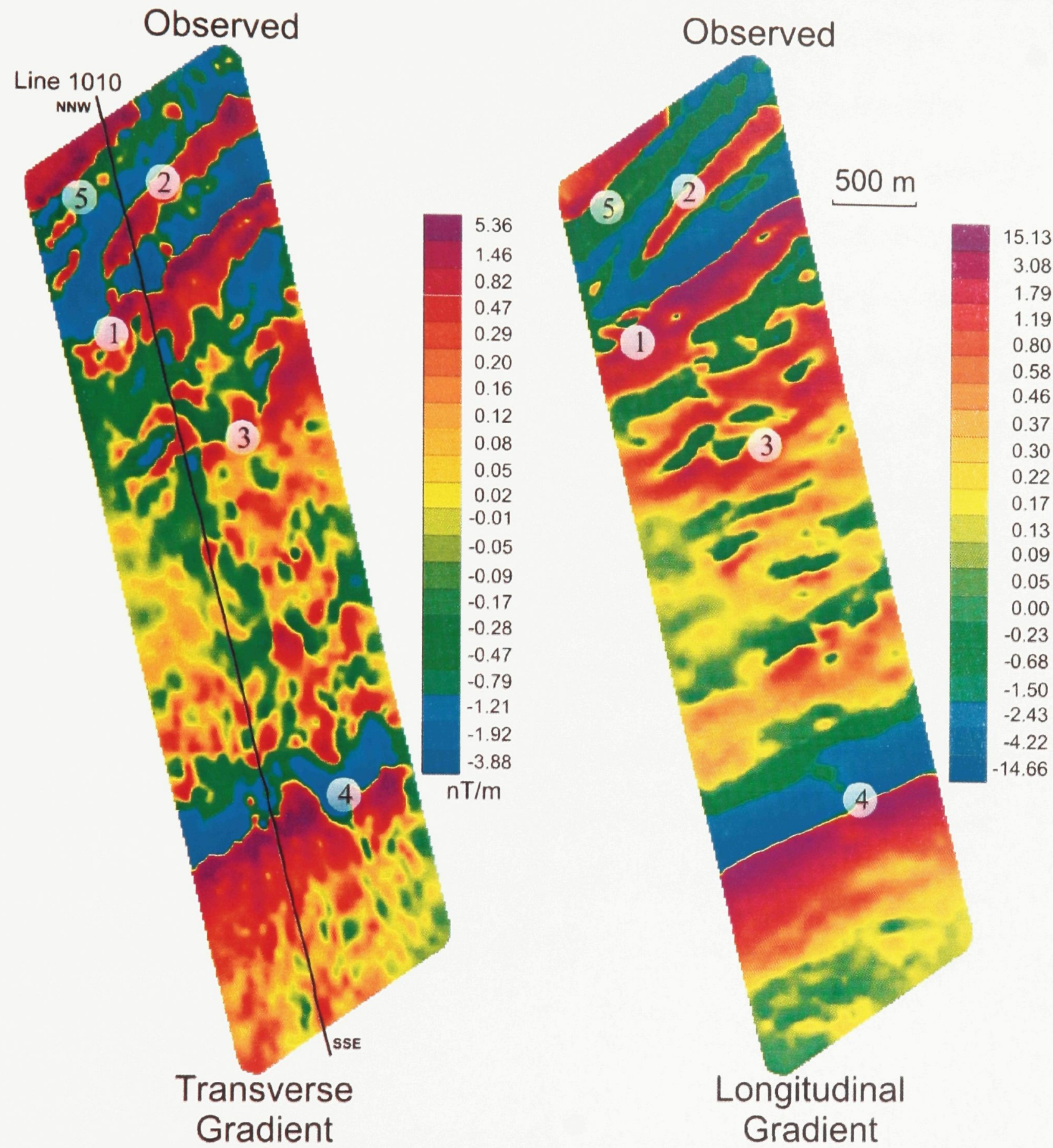

Figure 5.12

Comparison of the observed transverse gradient and the observed longitudinal gradient of the TMI map from the sUAS survey. 
While this case history has demonstrated the functionality of the transverse gradiometer at a nominal altitude of $50 \mathrm{~m}$ above ground, unknown is the maximum altitude at which the transverse gradiometer can make reliable measurements. Figure 5.13 is a comparison of observed transverse gradients measured by the sUAS along traverse line 1010 at different nominal altitudes above ground. The $50 \mathrm{~m}$ profile, shown by the purple line, represents the observed transverse gradient shown in figures 5.10 to 5.12. This profile has a higher frequency content, with larger amplitudes, compared to other profiles. The $100 \mathrm{~m}$ transverse gradient, shown in green, shows some attenuation in amplitude of the high frequency peaks, but is still able to reproduce most of the peaks seen in the $50 \mathrm{~m}$ profile. At a nominal altitude of $150 \mathrm{~m}$, shown in blue, the amplitudes of the high frequency peaks attenuate to the point that a large amount of detail is lost. The shape of individual peaks becomes distorted as they begin to fuse with adjacent peaks. The transverse gradient profile measured at a nominal altitude of $200 \mathrm{~m}$, shown in red, has little in common with the $50 \mathrm{~m}$ transverse profile. At this altitude only the most prominent peaks are reproduced. This profile may not have the resolution required to identify smaller magnetic trends.

Visual inspection of the observed gradient profiles along line 1010 plotted in Figure 5.13 shows that all trends can be recreated with the gradiometer flown at a nominal altitude of $100 \mathrm{~m}$ above the bedrock. At higher altitudes, such as at $150 \mathrm{~m}$, some trends are captured with distortion. Above the nominal altitude of $150 \mathrm{~m}$, only major trends are captured. This gives the $4.67 \mathrm{~m}$ gradiometer an operational limit, in the 


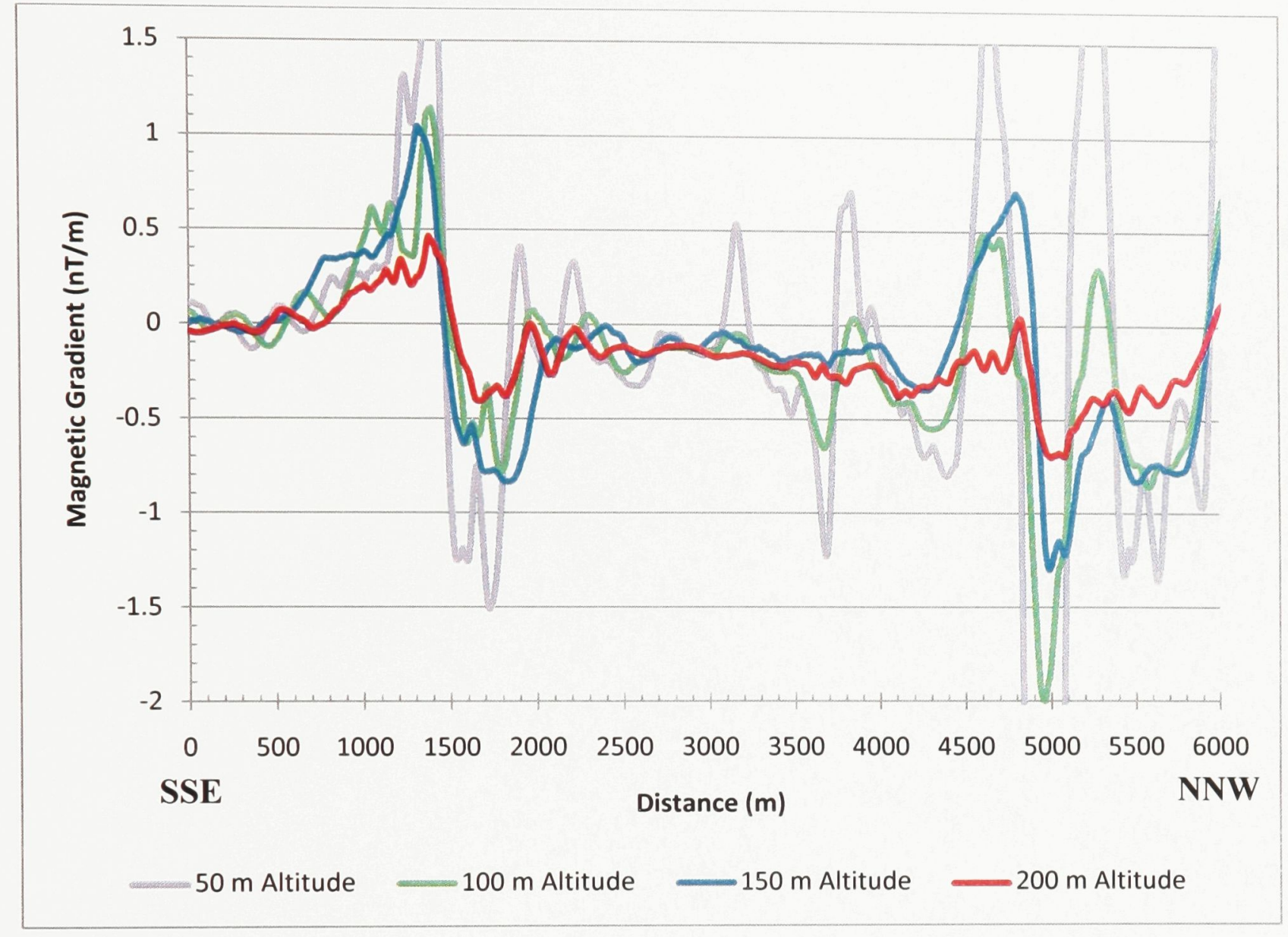

\section{Figure 5.13}

Comparison graph of observed transverse gradient measured at different altitudes. Measurements were taken during the sUAS survey while following a drape along traverse line 1010. The same drape was followed at each of the altitudes listed. 
geological setting of the Central Metasedimentary Belt of the Grenville province, of 100 $\mathrm{m}$ or less above the bedrock in order to measure high frequency magnetic trends. 


\section{Conclusion}

The primary objective of this thesis, which is to determine the ability of the sUAS to detect small magnetic details created from geological structures, can be answered by using the results shown in section 5.2 and 5.3. The comparison of the TMI maps created from the data gathered by the fixed wing, the ground survey, and the sUAS survey provide a quick, qualitative assessment of the ability of the sUAS at aeromagnetic surveying based on visual inspection. The results presented in section 5.2 show that the sUAS survey is able to create higher resolution TMI and FVD maps than the conventional fixed-wing survey, by flying at a lower altitude over the survey site. The sUAS TMI and FVD maps show greater detail in the strong magnetic trend of feature 1, and are also able to detect magnetic trends that are absent on the fixed-wing TMI and FVD maps, such as feature 5. Section 5.2 also shows that a survey does not need to be conducted at ground level in order to detect subtle geologically significant magnetic trends. The sUAS is able to delineate the magnetic trends that are detected during the ground survey. In addition to this, the sUAS survey is able to cover the area more thoroughly than the ground survey, and is able to create a more accurate TMI map, free from interpolation problems created from missing sections. The sUAS has proven to be a capable aeromagnetic surveying tool that is able to measure the magnetic field accurately. The increase in resolution shows that low altitude aeromagnetic surveying has much to offer towards a geological interpretation. 
The secondary objective of this thesis, to determine if the magnetic gradiometer configuration being designed for the GeoSurv II UAS is capable of recording the transverse gradient at low altitudes, is answered by the results shown in section 5.4. The measured gradient is able to reproduce all of the magnetic trends, which the derived gradient delineated, at a slightly higher level of detail. The measured transverse gradient is also in good agreement with the FVD and the derived longitudinal gradient. A profile comparison of the measured transverse gradient taken along line 1010 also shows that the gradiometer has detected all of the magnetic trends when flown at a nominal altitude of $100 \mathrm{~m}$ above the survey site. As the bedrock in the area is on average about $5 \mathrm{~m}$ below ground, the transverse gradiometer should be able to resolve most of the magnetic trends in a survey site if the survey is conducted at an average height of $100 \mathrm{~m}$ above the source rock. At a height above $100 \mathrm{~m}$ or less, the capability of the transverse gradiometer to resolve changes in the magnetic field over a distance of $4.67 \mathrm{~m}$ is decreased.

Some recommendations for future work would be to test the gradiometer configuration over a different type of terrain, where the bedrock is not as close to the surface. Testing the gradiometer configuration over a different type of geology such as with sedimentary rocks would be beneficial. Once the GeoSurv prototype has achieved flight status, the aircraft should be tested for any magnetic interference it may cause to recorded survey data. The figure of merit will need to be evaluated along with an analysis of methods that can possibly improve the figure through engineering modifications. The aircraft will then require an evaluation of its magnetic field. This is to ensure that the 
gradiometer measurements recorded by each magnetometer are not adversely effected in a different manner by the aircraft.

This thesis has demonstrated that the sUAS is able to gather a high resolution magnetic data set that is superior to a conventional fixed-wing data set, and comparable to a ground survey data set. Furthermore, this thesis has shown that a transverse gradiometer with a separation of $4.67 \mathrm{~m}$ can accurately delineate changes in the magnetic field when operated at a height that is approximately $100 \mathrm{~m}$ above the source rock. This thesis has also demonstrated through the use of the sUAS, that the design of the GeoSurv II UAS being developed by Carleton University and Sander Geophysics should allow the acquisition of accurate and high resolution magnetic and magneto-gradiometric data. 


\section{References}

Anderson, David E., and Pita, Alejandro C.. 2005 Geophysical Surveying with GeoRangerTMUAV. American Institute of Aeronautics and Astronautics (AIAA)

Ando, Shigeru. 1965. Shifts in Output Frequency of the Cesium Vapor Magnetometer due to Temperature, Light Intensity and Orientation. Japanese Journal of Applied Physics. Vol. 4, No. 10, p. 793-802

Ambrosia, V.G., Cobleigh, Brent, Jennison, Chris, and Wegener, Steve. 2007. Recent Experiences with Operating UAS in the NAS. American Institute of Aeronautics and Astronautics, 2007 Conference and Exhibit.

Allen, John, and Walsh, Brian. 2008. Enhanced Oil Spill Surveillance, Detection and Monitoring Through the Applied Technology of Unmanned Air Systems. International Oil Spill Conference: Research and Development, Savannah, Georgia, USA. p.113-120

Aviation Safety Unmanned Aircraft Program Office (ASUAPO) AIR-160. 2008. UAS Interim Operational Approval Guidance 08-01: Unmanned Aircraft Operations in the National Airspace System. Federal Aviation Administration, Department of Transportation 
Barnard, Joseph A.. 2008. The use of unmanned aircraft in oil, gas and mineral E+P activities. SEG Expanded Abstracts. Vol. 27, p. 1132

Bell, William E. and Bloom, Arnold L.. 1957. Optical Detection of Magnetic Resonance in Alkali Metal Vapor. Physical Review. Vol. 107, No. 6, p. 1559-1565

Billing, Stephen, and Wright, David. 2009. Optimal total field magnetometer configuration for near-surface applications. The Leading Edge. Vol. 28, No. 5, p. $522-527$.

Billingsley Aerospace \& Defense. 2008. TFM100-G2 Ultra Miniature Triaxial Fluxgate Magnetometer: February 2008 Specifications. Germantown. Maryland.

Bloom, Arnold L.. 1962. Principles of Operation of the Rubidium Vapor Magnetometer. Applied Optics. Vol. 1, No. 1, p. 61-68

Black, S.J., and Rencz, A.N.. 1987. Industrial Minerals in Eastern Ontario: Clarendon Sillimanite occurrence. Open File 1672. p.3.

Briggs, Ian C.. 1974. Machine Contouring Using Minimum Curvature. Geophysics. Vol. 39. No. 1, p. 39-48. 
Caron, R., Samson, C., Straznicky, P., Ferguson, S., Archer, R. and Sander, L. 2011. Magnetic and magneto-gradiometric surveying using a simulated unmanned aircraft system. Submitted and accepted to the 81 st Annual Meeting of the Society of Exploration Geophysicists, San Antonio, Texas, 18-23 September, Session "GM2 - Applications and Field Studies session".

Dehmelt, H.G.. 1957. Slow Spin Relaxation of Optically Polarized Sodium Atoms. Physical Review. Vol. 105, No. 5, p. 1487-1489

Easton, R.M., 2006a. Precambrian geology of the Cloyne-Plevna-Ompah area, northern Mazinaw Domain, Grenville Province: Ontario Geological Survey, Open File Report 5454.

Easton, R.M. 2006b. Precambrian geology, Cloyne-Plevna-Ompah area; Ontario Geological Survey, Preliminary Map P.3443, scale 1:50 000.

Gem Systems. 2008. GSM-19 v7.0 Instruction Manual. Richmond Hill, Ontario.

Geometrics, 2004. G-822A and G-823A \& B Cesium Magnetometer Operation Manual. Rev B. Geometrics, California.

Geophex Limited, 1996. Geophex Airborn Unmanned Survey System (GAUSS); Topical Report October 1993 - September 1996. Geophex, North Carolina. 
Grotewahl, Max. 1930. Bericht Über die Versuchsfahrt des Bidlingmaierschen Doppelkompasses mit dem Luftschiff "Graf Zeppelin". Terr. Magn. Vol. 35 p.226-229.

Hardwick, C. D., 1984. Important design considerations for inboard airborne magnetic gradiometers. Geophysics, Vol. 49, p. 2004-2018.

Holland, J.G. 1876. The Century illustrated monthly magazine, Vol. 12. Scribner \& Co. New York. p. 445

Holland, Greg J., McGeer, Tad, and Youngren, Harold. 1992. Autonomous Aerosondes for Economical Atmospheric Soundings Anywhere on the Globe. Bulletin of the American Meteorological Society. Vol. 73, p. 1987-1998

Holland, G J., Webster P.J., Curry, J.A., Tyrell, G., Gauntlett, D., Brett, G., Becker, J., Hoag, R., and Vaglienti, W., 2001. The Aerosonde Robotic Aircraft: A New Paradigm for Environmental Observations. Bulletin of the American Meteorological Society, Vol. 82, No. 5, p. 889-901

Honeywell, 2011. Honeywell T-Hawk Aids Fukushima Daiichi Disaster Recovery: Unmanned Micro Air Vehicle Provides Video Feed to Remote Monitors. Honeywell Press Releases, April $19^{\text {th }} 2011$. 
Hood, P. J., and D.J. Teskey, 1989. Aeromagnetic Gradiometer Program of the Geological Survey of Canada: Geophysics, Vol. 54, p. 1012-1022.

Hood, Peter. 2007. History of aeromagnetic surveying in Canada. The leading Edge. Vol. 26, No. 11, p. 1384-1392

Hrvoic, Ivan., Hollyer, Greg M. 2002. Brief Review of Quantum Magnetometers. Gem Systems. Richmond Hill, Ontario.

Keiswetterg, Dean, and Novikova, Elena. 1996. Geophex airborne unmanned survey system (GAUSS). SEG Expanded Abstracts. Vol.15, p.944

Leliak, Paul. 1961. Identification and Evaluation of Magnetic-Field Sources of Magnetic Airborne Detector Equipped Aircraft. Aerospace and Navigational Electronics: IRE Transactions of Aerospace and Navigational Electronics. Vol. 8, No. 3, p. 95105.

Lundak, Asger. 1954. About Possibilities and Limitations in Aeromagnetic Surveying. Pure and Applied Geophysics. Vol. 27, No. 1, p. 110-115

Macnae, James. 1995. Design specifications for a geophysical unmanned air vehicle assembly (GUAVAS). SEG Expanded Abstracts. Vol.14, p.375 
Mahendran, Mario. 2010. An Improved Mouldless Manufacturing Method for FoamCore Composite Sandwich Structures: MSc thesis, Carleton University.

Maley, J. A.. 2008. An investigation into low-cost manufacturing of carbon epoxy composites and a novel "mouldless" technique using the Vacuum Assisted Resin Transfer Moulding (VARTM) method: MSc thesis, Carleton University.

McMullan, S.R., and McLellan, W.H.. 1997. Measured is better. Applications of Regional Geophysics and Geochemistry, Paper 116. In "Proceedings of Exploration 97: Fourth Decennial International Conference on Mineral Exploration" edited by A.G. Gubins, p. 873-876

Muffly, Gary. 1946. The Airborne Magnetometer. Geophysics, vol. 11 p. 321-334.

Mushayandebvu, M.F., and Davies, J.. 2006. Magnetic gradients in sedimentary basins: Examples from the Western Canada Sedimentary Basin. The Leading Edge. Vol. 25, No. 1, p. 69-73.

Noll, Thomas, Brown, J., Perez-Davis, M., Ishmael, S., Tiffany, G., and Gaier, M.. 2004. Investigation of the Helios Prototype Aircraft Mishap, Volume I Mishap Report. NASA. 
O’Connell, Michael D., Smith, Richard S., and Vallée, Marc A.. 2005. Gridding aeromagnetic data using longitudinal and transverse horizontal gradients with the minimum curvature operator. The Leading Edge. Vol. 24, No. 2, p. 142-145.

Office of the Secretary of Defense. 2005. Unmanned Aircraft Systems Roadmap 20052030. United States of America Department of Defense.

Ontario Geological Survey, 2003. Ontario airborne geophysical surveys, magnetic data, Kaladar-Marmora area: Ontario Geological Survey, Geophysical Data Set 1018 Revised.

Paine, J. W.. 1986. A Comparison of Methods for Approximating the Vertical Gradient of One-dimensional Magnetic Field Data. Geophysics. Vol. 51, No. 9, p. 17251735.

Pauk, L.. 1982. Geology of the Ardoch area, Frontenac county. Ontario Geological Survey, Open File Report 5381. P.71.

Reeves, Colin. 2005. Aeromagnetic Surveys: Principles, Practice \& Interpretation. Epublished by GeoSoft Incorporated, 2005.

Reynolds, John M.. 1997. An Introduction to Applied and Environmental Geophysics. John Wiley \& Sons. Toronto. p.117 
Saggiani, G., Persiani, F., Ceruti, A., Tortora, P., Troiani, E., Giuletti, F., Amici, S., Buongiorno, M., Distefano, G., Bentini, G., Bianconi, M., Cerutti, A., Nubile, A., Sugliani, S., Chiarini, M., Pennestri, G., Petrini, S., and Pieri, D.. 2007. A UAS System for Observing Volcanoes and Natural Hazards. American Geophysical Union, Fall Meeting 2007, abstract \#GC11B-05

Samson, C., Straznicky, P., Laliberté, J., Caron, R., Ferguson, S., and Archer, R.. 2010. Designing and building an unmanned aircraft system for aeromagnetic surveying: 80th Annual International Meeting, SEG, Expanded Abstracts, p.1167-1171.

Sander, Luise and Moul, Francis. 2004. How much noise can we tolerate in horizontal gradiometer data? Society of Exploration Geophysicists - Workshop on Magnetic Gradient, Denver, October 2004

Schwing, Lt. Colonel Richard. 2007. Unmanned Aerial Vehicles - Revolutionary Tools in War and Peace. USAWC Strategy Research Project.

Smith, Matt. 2011. Flying drone peers into Japan's damaged reactors. CNN, April $10^{\text {th }}$ 2011.

Telford, W.M., Geldart, L.P., Sheriff, R.E., and Keys, D.A.. 1990. Applied Geophysics, 2nd edition. Cambridge University Press. p. 62 
Thalén, 1879. The examination of Iron Ore Deposits by Magnetic Measurements

Transport Canada. 2010. Canadian Aviation Regulations (CARs). Transport Canada. Section $101.01,602.41$

Transport Canada. 2007. Civil Aviation Unmanned Air Vehicle Working Group Final Report. Transport Canada.

Warry, Richard. 2011. Q\&A: Health effects of radiation exposure. BBC News, April $18^{\text {th }}$ 2011.

Waters, G.S. and Frances, P.D.. 1958. A nuclear magnetometer. Journal of Scientific Instruments, vol. 35 p. 88 